NOTE TO USERS

This reproduction is the best copy available.

(B)

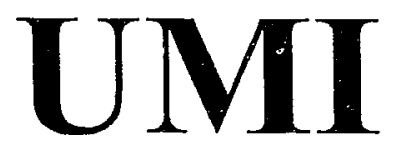

Reproduced with permission of the copyright owner. Further reproduction prohibited without permission. 
Reproduced with permission of the copyright owner. Further reproduction prohibited without permission. 


\title{
FATIGUE ANALYSIS OF FUEL TANK STRAPS DUE TO SLOSH LOADS
}

\author{
by \\ ALIREZA FEREIDOONI, \\ B.Sc. (Mechanical Engineering) \\ K.N.T. University of Technology, 1988
}

\author{
A thesis \\ presented to Ryerson University \\ in partial fulfillment of the requirement for the degree of \\ Master of Applied Science \\ in the Program of \\ Mechanical Engineering
}

Toronto, Ontario, Canada, 2004

(C) Alireza Fereidooni, 2004

PROPERTY DF

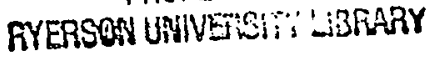

Reproduced with permission of the copyright owner. Further reproduction prohibited without permission. 
UMI Number: EC52952

\section{INFORMATION TO USERS}

The quality of this reproduction is dependent upon the quality of the copy submitted. Broken or indistinct print, colored or poor quality illustrations and photographs, print bleed-through, substandard margins, and improper alignment can adversely affect reproduction.

In the unlikely event that the author did not send a complete manuscript and there are missing pages, these will be noted. Also, if unauthorized copyright material had to be removed, a note will indicate the deletion.

(®)

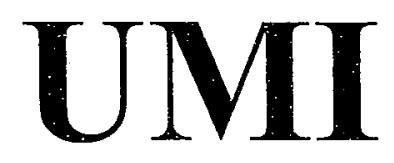

UMI Microform EC52952

Copyright 2009 by ProQuest LLC.

All rights reserved. This microform edition is protected against unauthorized copying under Title 17, United States Code.

ProQuest LLC

789 E. Eisenhower Parkway PO Box 1346

Ann Arbor, MI 48106-1346 


\author{
ABSTRACT \\ (C) Alireza Fereidooni, 2004 \\ Master of Applied Science \\ in the Program of \\ Mechanical Engineering \\ Ryerson University
}

FATIGUE ANALYSIS OF FUEL TANK STRAPS DUE TO SLOSH LOADS

A great number of investigations on fatigue analysis and liquid sloshing has been carried out in different fields of application such as space vehicles, large ground tanks, cargo ships, and highway truck tankers. A variety of approaches to solve the sloshing problem due to different requirements, tank characteristics, and peculiarities of input disturbance gives different, sometimes contradictory, results which are not directly applied to the car fuel tank system.

One of the most important components in the fuel tank system is strap, which has vital role in road safety. Fuel tank strap can be separated due to fatigue during vehicle operation, causing the tank to be unsupported and increasing the risk of fuel leak. Its failure can cause very dangerous accidents. The effect of sloshing load, one of the main applied loads on the tank structure, on life of the strap has not been studied carefully before.

This thesis presents the numerical analysis of liquid behaviour in sloshing situation and the fatigue analysis of the fuel tank straps due to slosh loads exerted on the tank walls in an accelerating car. In this study, computational fluid dynamics (CFD) and finite element method (FEM) are employed to simulate transient sloshing of fuel inside the tank and find 
the location of the critical point of crack initiation and predict the life of the fuel tank strap. Using the volume of fluid (VOF) method, a new developed method in CFD, the range of loads on the walls of the fuel tank is determined for the operational conditions that the vehicle experience. An equivalent mechanical model is developed to verify the CFD sloshing simulation result through the normal impact load applied by the effective mass of a pendulum system.

The finite element (FE) is further used to create the virtual model of the strap and perform a static analysis. The stresses and strains from the static analysis are then used as input to locate the critical point and predict the life expectancy of the strap under slosh loading. 


\section{ACKNOWLEDGEMENTS}

I would like to express my sincerest gratitude to my supérvisors, Professor Kamran I.

Behdinan and Professor Zouheir Fawaz who have been very supportive, and I wish to thank them for their valuable guidance, scientific suggestions as well as their contributions to this research.

Also I am indebted to my friendly colleagues at the ATOP,Advanced Computational Laboratory of Ryerson University, for their help and priceless support in the Finite Element Modeling and Analysis aspect of this research. 


\section{DEDICATION}

I would like to dedicate this thesis to my son and my daughter, Saman and Sonia. 


\section{TABLE OF CONTENTS}

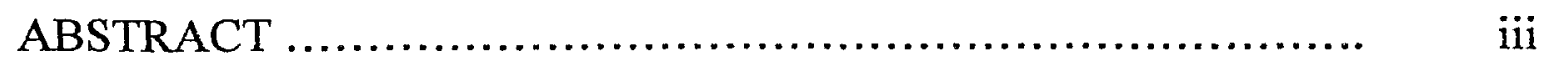

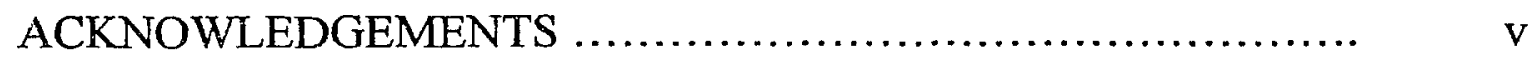

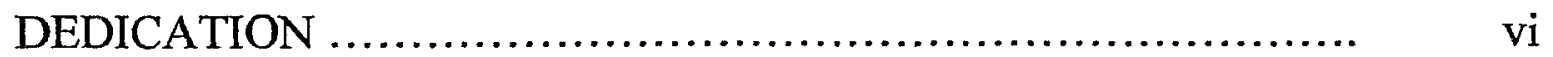

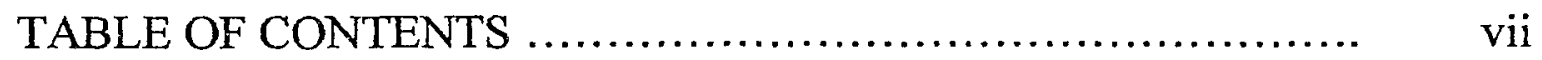

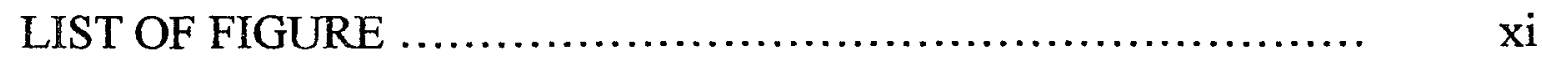

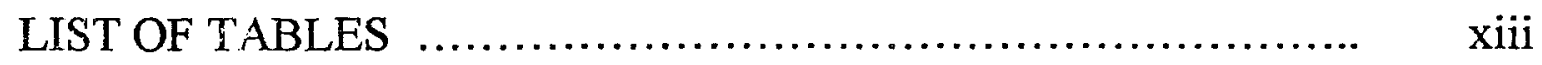

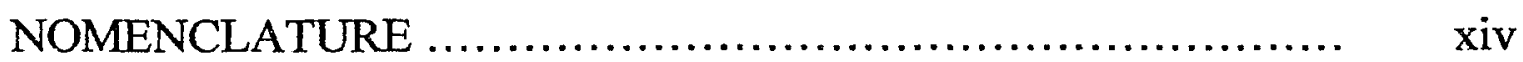

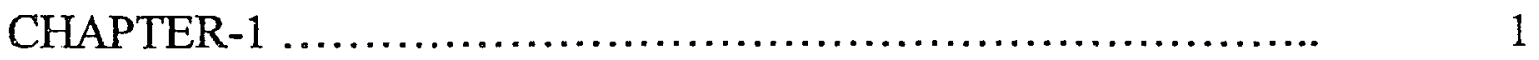

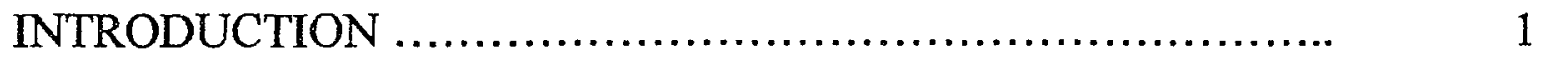

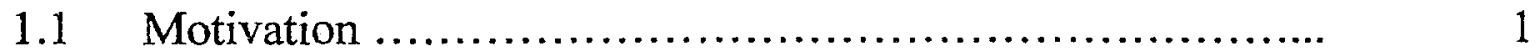

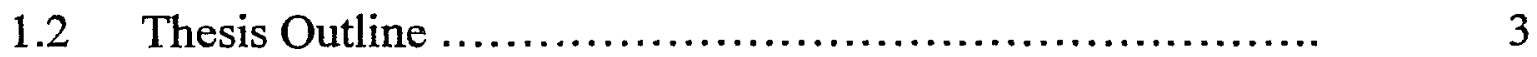

CHAPTER -2 ............................................. 5

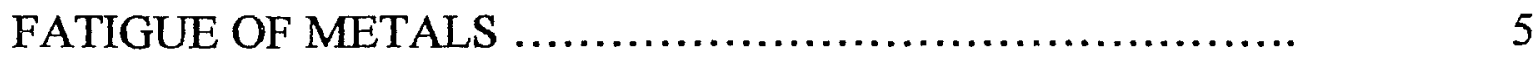

$2.1 \quad$ Introduction........................................... 5

$2.2 \quad$ Stress Cycles.........................................

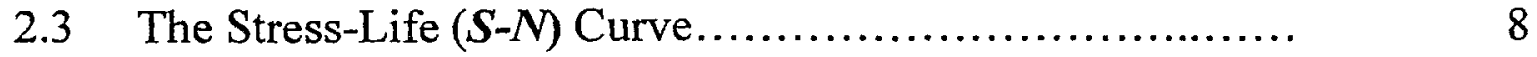

vii 
$2.4 \quad$ Effect of Mean Stress on Fatigue.......................... 10

2.5 The Strain-Life $(\varepsilon-N)$ Curve........................... 11

2.6 Cyclic Stress-Strain Curve.............................. 13

$2.7 \quad$ Design for Fatigue.....................................

2.8 Application of Finite Element Analysis................... 22

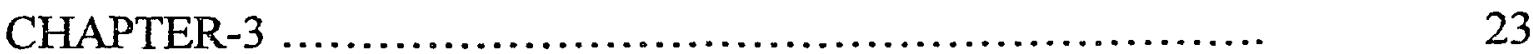

SLOSHING SIMULATION .................................... 23

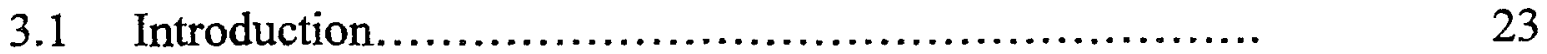

3.2 Volumeof Fluid Method and Finite Element ...............

3.3 Finite Element Modeling of Fuel Tank................... $\quad 28$

$3.4 \quad$ Sloshing Analysis . ................................. 30

3.4.1 Introduction ...........................................

3.4.2 Overview of Numerical Scheme ............................ 32

3.4.3 Segregated Solution Algorithm (SSA) …................

3.4.4 Coupled Splution Algorithm (CSA) .....................

3.4.5 Linearization: Implicit vs. Explicit ......................

3.4.6 Under Relaxation Factor ................................

3.4.7 Material Properties....................................... 37

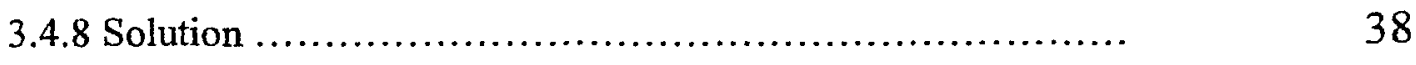

3.5 Sloshing Simulation Results ........................ 40

viii 


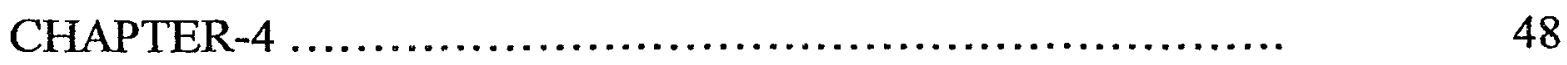

EQUTVALENT MECHANICAL MODELS FOR LIQUID SLOSHIIIG SIMULATION ......................................................

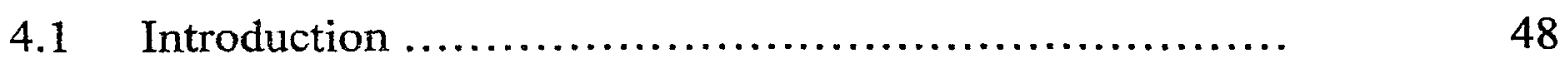

4.2 Sloshing Forces Analysis .............................

4.3 Mechanical Analysis of Liquid Sloshing .................. 50

4.4 Pendulum Model for Liquid Sloshing in Rectangular Tanks 50

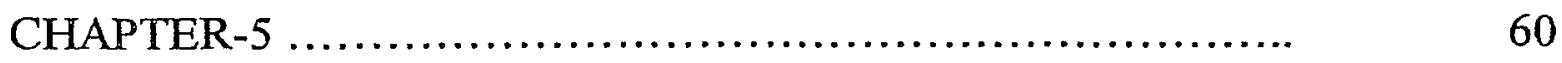

FATIGUE ANALYSIS OF FUEL TANK STRAPS............... 60

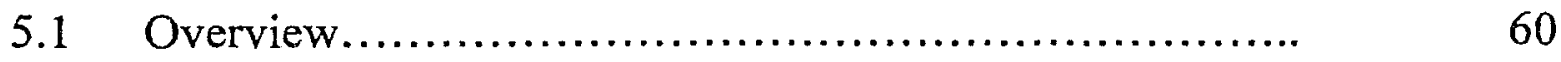

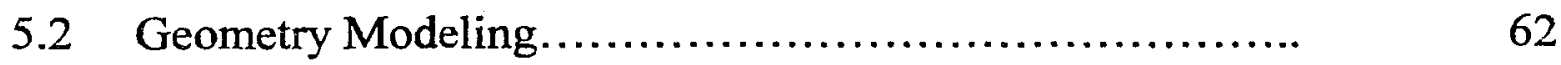

5.3 Finite Element Discretizing............................ 63

5.4 Boundary Conditions.................................. 74

5.5 Finite Element Static Analysis: Analysis Parameter........ 67

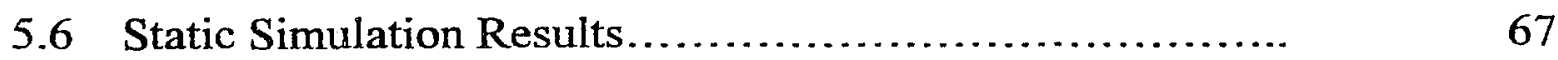

5.7 High Cycles vs. Low Cycles Domains .................... 68

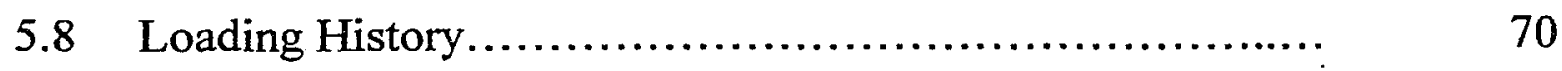

ix 
5.9 Fatigue Life Estimation of the Strap..................... 71

5.10 Simuation Results ...................................... 71

5.11 Results Summary ................................. 72

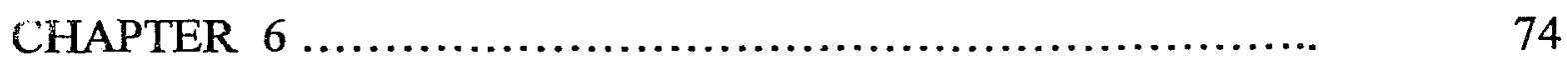

CONCLUSION AND RECOMMENDATIONS ................ 74

6.1 Conclusion............................................ 74

6.2 Recommendations for Future Work ..................... 75

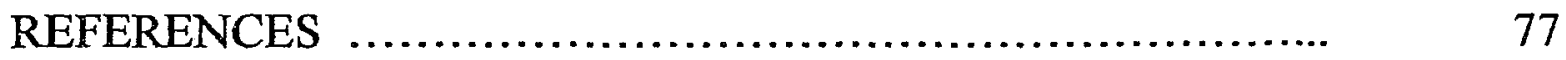

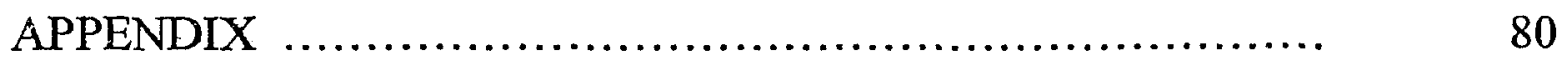




\section{LIST OF FIGURES}

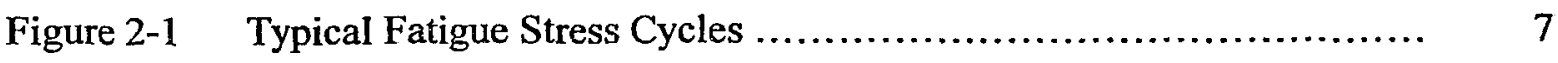

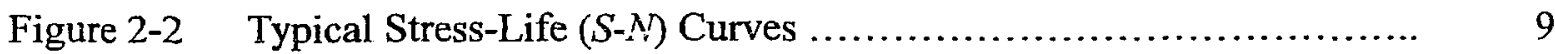

Figure 2-3 Typical Strain-Life $(\varepsilon-N)$ Curve............................... 12

Figure 2-4 Typical Stress-Strain Curve .................................. 14

Figure 2-5 A Comparison between True and Engineering Stress-Strain Curves 14

Figure 2-6 Loading Reversed into Compression .......................... 15

Figure 2-7 A Complete Stress-Strain Cycles, a Hysteresis Loop ............... 16

Figure 2-8 Cyclic Softening and Hardening under Strain Control ................ 17

Figure 2-9 The Cyclic Stress-Strain Curve ............................... 18

Figure 2-10 A Comparison between Cyclic and Monotonic Stress-Strain Curves 19

Figure 3-1 Typical Pattern of the Free Surface of Flow in a Discretized Tank 26

Figure 3-2 Typical Pattern of the actual Free Surface Interface and Donor-Acceptor

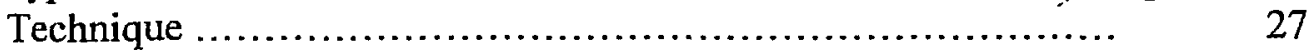

Figure 3-3 Schematic Drawing of the Fuel Tank with three Baffles ............ 28

Figure 3-4 CAD Model of Fuel Tank ................................... 29

Figure 3-5 Fuel Tank Assembly meshed in GAMBIT .................... 30

Figure 3-6 Imported Discretized Model of the tank to FLUENT ............. 31

Figure 3-7 Overview of the Segregated Solution Algorithm ................. 33

Figure 3-8 Overview of the Coupled Solution Algorithm ................... 35

Figure 3-9 Material Properties of two Phases inside the Tank ................ 37

Figure 3-10 The Pressure Profile snapshot of FE Simulation of shallow, intermediate, and deep Fuel Sloshing in the Baffled Tanks ..................... 41

$\mathrm{xi}$ 
Figure 3-11 The Fuel Locations snapshot of FE Simulation of shallow, intermediate, and deep Fuel Sloshing in the Baf._. - d Tanks

Figure 3-12 Comparison between the exerted Slosh Forces on the Tank Wall in the $\mathrm{x}$-direction for $25 \%-50 \%$ - and $75 \%$ of the Tank volume filled with Fuel

Figure 3-13 Sloshing Pressure Profiles for Baffled and Un-baffled Tanks........

Figure 3-14 Fuel Locations for Baffled and Un-baffled Tanks................. 46

Figure 3-15 Comparison of Slosh Forces in baffled and un-baffled tanks.........

Figure 4-1 Liquid motions in moving Tanks

Figure 4-2 Three regimes of Liquid Free Surface motion and their Modeling ....

Figure 4-3 Schematic diagram of Pendulum Model on elastic structure

Figure 4-4 A lateral exited Tank and a Pendulum Model

Figure 4-5 Comparison of Impact Forces exerted on the Wall of the Rectangular Tank obtained from FE Model and Pendulum Model

Figure 5-1 Strap Geometry Model

Figure 5-2 Discretized Model of the Strap

Figure 5-3 Maximum Slosh Loads applied on the Strap for static analysis ...... 66

Figure 5-4 Applied Loads in the z-direction on the Strap and Fixed Nodes around the holes

Figure 5-5 Maximum Principal Stress contours obtained from Static Analysis of the Strap

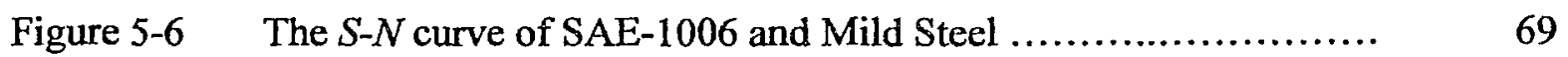

Figure 5-7 Loading History obtained from Sloshing Simulation.............. 70

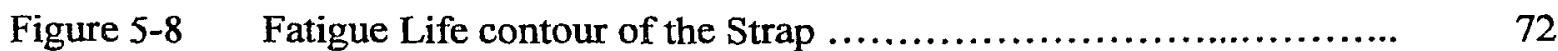

Figure A-1 Pendulum Model for Cylindrical Tank ......................... 80

Figure A-2 Radial Force exerted on the Tanker during Turning ............... 82 


\section{LIST OF TABLES}

Table 3-1 Slosh Forces on the Wall of the Fuel Tank of an Accelerating Car for $25 \%-50 \%$-and $75 \%$ fuel depths ............................

Table 5-1 Typical Element Types and their Applications $\quad . . . . \ldots \ldots \ldots \ldots \ldots . . . . . . .$.

xiii

Reproduced with permission of the copyright owner. Further reproduction prohibited without permission. 


\section{NOMENCLATURE}

a: $\quad$ tank width

atm: atmosphere

$a_{x}: \quad$ lateral acceleration

A: $\quad$ Amplitude ratio

$b: \quad$ tank length

B: $\quad$ mass matrix

D: damping coefficient matrix

d: $\quad \operatorname{tank}$ depth

$E: \quad$ elastic modulus

$f: \quad$ frequency

$F: \quad$ volume fraction (function)

$F_{I}: \quad$ slosh impact load

g: $\quad$ gravity in z-direction

h: $\quad$ tank height

$K^{1}: \quad$ cyclic strength coefficient

$K_{t}: \quad$ stress concentration factor

$k$ : torsional spring stiffness

K: $\quad$ stiffness matrix

Kpa: kilo pascal

$l$ : length of pendulum

$L: \quad$ length of elastic structure rod

$m: \quad$ mass of pendulum

xiv

Reproduced with permission of the copyright owner. Further reproduction prohibited without permission. 
M. mass of container

Mpa: mega pascal

$n$ : normal co-ordinate

n: normal vector

$\mathrm{n}$ : number of cycles of operation

$n^{1}$ : cyciic strain hardening exponent

$N: \quad$ total number of cycles of operation

$N_{f}$. $\quad$ total number of cycles to failure

N: Newton

$P: \quad$ pressure

Pa: pascal

$\mathrm{r}, \mathrm{R}: \quad$ radius

$\boldsymbol{R}: \quad$ Stress ratio

$S: \quad$ engineering stress

$S_{a}$ : allowable engineering stress amplitude

$S_{m}: \quad$ mean engineering stress

$S_{u}: \quad$ ultimate tensile stress

$S_{\text {max: }} \quad$ maximum engineering waress

$S_{\min }: \quad$ minimum engineering stress

$S_{r}: \quad$ range of stress

$S_{o}: \quad$ allowable engineering stress amplitude at zero mean stress

$S_{u}: \quad$ ultimate engineering tensile stress

$t: \quad$ time 
T: $\quad$ kinetic energy

$u_{x}: \quad$ velocity component in $\mathrm{x}$-direction

w: velocity vector

$\mathrm{U}$ : potential energy

$\mathrm{V}: \quad$ volume

$\mathrm{w}$ : velocity component in $\mathrm{z}$-direction

$\mathrm{x}, \mathrm{y}, \mathrm{z}$ cartesian co-ordinates

$x_{p}, x:$ pendulum position in $\mathrm{x}$-direction

$x_{c}: \quad$ container position in $\mathrm{x}$-direction

$z_{p}, z: \quad$ pendulum position in z-direction

$z_{\mathrm{c}}: \quad$ container position in $\mathrm{z}$-direction

\section{Greek}

$\varepsilon_{\imath} \quad$ total strain

$\varepsilon_{f}^{1} \quad$ fatigue ductility coefficient

$\sigma \quad$ true stress

$\sigma_{f} \quad$ true fracture strength

$\sigma_{f}^{1} \quad$ fatigue strength coefficient

$\sigma_{\max }$ maximum true stress

$\sigma_{0} \quad$ Morrow mean stress

$\sigma_{x} \quad$ maximum principal stress in the x-direction

$\sigma_{y} \quad$ maximum principal stress in the y-direction

xvi 
$\sigma_{z} \quad$ maximum principal stress in the $\mathrm{z}$-direction

$\theta: \quad$ pendulum angle with vertical axes

$\omega: \quad$ frequency

$\varphi$ : $\quad$ massless rod angle with vertical axes

$v$ ratio of frequency

$\omega_{L}, \omega_{R}:$ frequency of tank

$\omega_{l}: \quad$ frequency of pendulum

$\mu: \quad$ viscosity

$\rho: \quad$ density

$\nabla: \quad$ gradient operator 


\section{CHAPTER-1 INTRODUCTION}

\subsection{Motivation}

In automotive industries, numerical optimization of vehicle components is an important aspect of producing high quality vehicles in a very competitive market. Due to the general trend of producing lighter and safer vehicles, it has become imperative to consider the durability of the structure being optimized. This can be achieved by finding the optimum thickness distribution for a minimum specified fatigue life value as an extra constraint in the optimization process [1].

In many areas of engineering, metallic structures are subjected to complex loads. One of these complex loads is the sloshing load caused by liquid motion in transporting containers. Sloshing is a very important problem especially if it is related to toxic, flammable, and other dangerous liquids [2]. Liquid sloshing constitutes a broad class of problems of great practical importance with regard to the safety of transportation systems, such as car fuel tanks on roads, fuel tanker trucks on highways, liquid tank cars on railroads, and sloshing of liquid cargo in oceangoing vessels. The extensive statistical data collected by the U.S. National Transportation Safety Board (NTSB) and the U.S. National Highway Traffic Safety Administration (NHTSA) give a clear view of the seriousness of the problem [3,4].

One of the vehicle components that have attracted the attention of engineers and transportation safety organizations is the fuel tank strap. Fuel tank straps should be strong enough to withstand such sloshing loads for a long time, otherwise their failure can cause 
very dangerous, harmful, and dramatic accidents. Road safety administrations are very sensitive to this matter. For example, the NHSTA issued the recall of 150,000 vehicles, model Dodge Durango (1998-99), in December 2000, because the "fuel tank strap could be separated due to fatigue during vehicle operation, causing the tank to be unsupported and increasing the risk of fuel leaks" [4]. Therefore fatigue analysis and life estimation of the fuel tank straps should be considered strongly as playing a vital role in road safety.

Fatigue failure is a multi-stage process that begins with crack initiation, propagating with continued cyclic loading and finally, rupture of the components [5]. As the components become more complex, the more difficult it is to apply analytical techniques to predict fatigue life. Hence, numerical approaches have been developed that make it possible to effectively analyze complex structures and obtain solutions faster $[1,5]$. One of the popular numerical approaches is the finite element method (FEM) that allows designers and engineers to create virtual models of components and perform simulations in the preliminary design stage.

The objective of this thesis is the fatigue life estimation of fuel tank straps due to sloshing loads exerted on the tank of an accelerating car. The FEM as a numerical tool is used to create the virtual model of the tank and straps and perform a static analysis. The stresses and strains from the static analysis are then used as input to locate the critical point and predict the life expectancy of the straps under slosh loadings [5].

In order to determine sloshing loads, liquid motion behaviour and free surface flows in a moving partially filled tank need to be analyzed. Some specified numerical techniques in computational fluid dynamics (CFD) such as volume of fluid (VOF) are developed to 
simulate sloshing. Sloshing presents free surface elevation or amplitude of the slosh, which in general, depends on the amplitude and frequency of the tank motion, liquid-fill depth, liquid properties and tank geometry. When the frequency of the tank motion is close to one of the natural frequencies of the liquid, large slos:ing amplitudes can be expected. The large liquid movement creates highly localized impact loads on tank walls that may cause structural damage of the tank including fuel tank straps and even create sufficient moment to affect the stability of the vehicle carrying containers $[7,8,9]$.

Due to the complexity of sloshing simulation in moving tanks, some assumptions have been considered. The fuel is assumed to be homogenous and remain laminar, isotropic, viscous and incompressible. The domain is assumed to be a rigid rectangular tank, which is partially filled with liquid fuel. These lead to a significant simplification of the momentum and mass equations with neglecting viscous term, and combining the resulting equations with the continuity equation [7].

Since sloshing behaviour depends on the container shape and internal baffles, testing becomes a very expensive task when studying the numerous design scenarios [10]. The equivalent mechanical model such as pendulum is reported to simulate and verify the results obtained from numerical simulation.

\subsection{Thesis Outline}

The contents of this thesis are organized as follows:

Chapter two provides the theoretical approaches on fatigue of metals including a brief explanation of the stress cycles, the stress-life $(S-N)$ curve, the strain-life $(\varepsilon-N)$ curve, 
the effect of mean stress on fatigue, the cyclic stress-strain curve, and the low cycle- high cycle fatigue.

Chapter three focuses on sloshing simulation in moving baffled and un-baffled fuel tanks using the VOF numerical method in CFD. This numerical method is explained in the commercial packages, GAMBIT and FLUENT, which are employed to make and discretize the Computer Aided Design (CAD) model of the fuel tank and then simulate the sloshing. The obtained results of slosh impact loads for $25 \%-50 \%$ and $75 \%$ of fuel depth for the same boundary conditions are graphed and illustrated.

Chapter four presents two equivalent mechanical models for liquid sloshing simulation including: 1- a pendulum model for rectangular tanks, 2- a pendulum model for cylindrical tanks. Also the numerical results obtained from FEM and mechanical models are graphed and compared.

In Chapter five a description of FEM modeling of the fuel tank strap using the commercial package MSC-PATRAN and NASTRAN is presented [5,11]. Static simulation with applying the boundary conditions and later on fatigue analysis of the strap due to slosh load-time history are explained in details. The fatigue life estimation of straps is presented in contour at the end of the Chapter.

Finally, Chapter six gives a brief discussion and some recommendations on future work. 


\section{CHAPTER-2 FATIGUE OF METALS}

This research presents the fatigue life estimation of fuel tank straps that are subjected to complex liquid slosh impact loads. It is important to understand the fundamental theories on fatigue failure behaviour. Hence this chapter focuses on both the basic and advanced forms of fatigue failure in metal structures.

\subsection{Introduction}

It has been recognized since 1830 that a metal part subjected to repetitive or fluctuating stress fails at a stress level much lower than that required to cause fracture by a single applied load [11]. Failures occurring under conditions of dynamic loading are called fatigue failures, presumably because it is generally observed that these failures occur only after a considerable period of service. This type of failure has become progressively more prevalent for the advancement of technology has led to a greater amount of equipment, such as automobiles, aircraft, compressors, pumps, turbines, etc. that are subjected to various types of repeated loading and vibration. It is currently reported that fatigue accounts for at least $90 \%$ of all service failures due to mechanical causes $[12,13]$.

A fatigue failure is particularly insidious because it occurs without any obvious warning. Fatigue results in a brittle-appearing fracture, with no gross deformation at the fracture. On a macroscopic scale, the fracture surface is usually normal to the direction of the principal tensile stress [12]. 
Three basic factors are necessary to cause fatigue failure $[11,12,13]$. These are

$>$ a maximum tensile stress of sufficiently high value

$>$ a large enough variation or fluctuation in the applied stress

$>$ a sufficiently large number of cycles of the applied stress.

Each of these factors will be discussed in the next sections. In addition, there are a host of other variables, such as stress concentration, corrosion, temperature, overload, metallurgical structure, residual stresses, and combined stresses, which tend to alter the conditions for fatigue.

\subsection{Stress Cycles}

At the outset, it is advantageous to define briefly the general types of fluctuating stresses that can cause fatigue. Figure 2-1 serves to illustrate typical fatigue stress cycles. Figure 2-1(a) illustrates a completely reversed cycle of stress of sinusoidal form. This is an idealized situation that is produced by the R. R. Moore rotating-beam fatigue testing machine using a rotating shaft with constant speed and without overloads [11]. For this type of stress cycle the maximum and minimum stresses are equal. The minimum stress is the lowest algebraic stress in the cycle. Tensile stress is considered positive, and compressive stress is negative $[12,13,14]$.

Figure 2-1(b) illustrates repeated stress cycles in which the maximum stress $S_{\max }$ and minimum stress $S_{\min }$ are not equal. They are both tensions in this illustration, but a repeated stress cycle could contain maximum and minimum stresses of opposite signs or both in compression. 

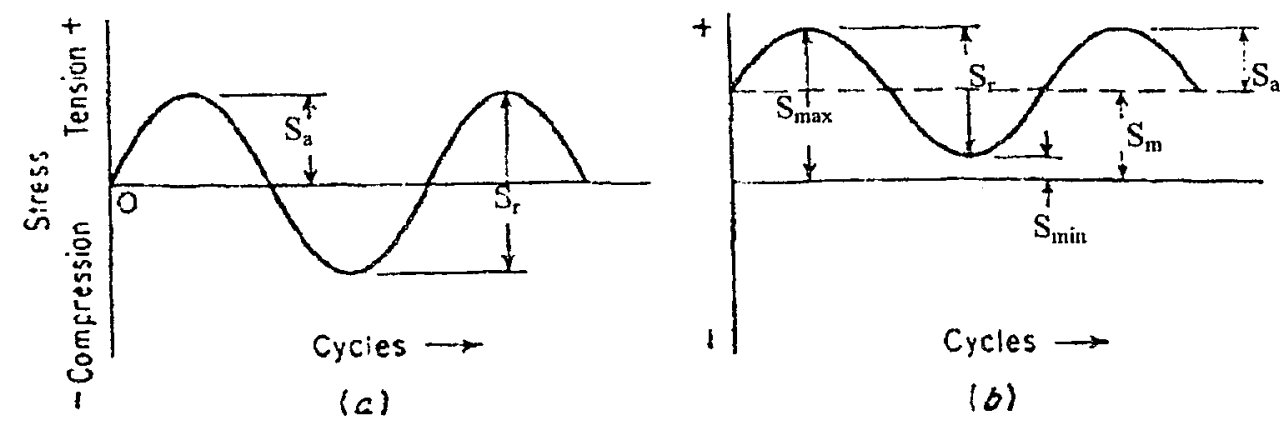

(b)

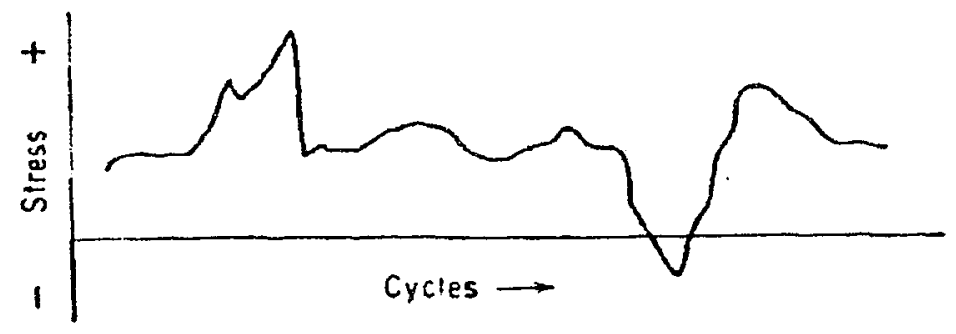

(c)

Figure 2-1 Typical Fatigue Stress Cycles. (a) Reversed Stress; (b) Repeated Stress; (c) Irregular or Random Stress Cycle [11].

Figure 2-1(c) illustrates a complicated and irregular stress cycle that may be encountered in a part such as an aircraft wing subjected to unpredictable overloads or in fuel tank straps subjected to irregular slosh impact loads $[13,14,15]$.

A fluctuating stress cycle can be made up of two components, a mean or steady stress $S_{m}$, and an alternating or variable stress $S_{a}$. The mean stress is the algebraic mean of the maximum and minimum stress, $S_{\max }$ and $S_{\min }$, in the cycle.

$$
S_{m}=\frac{S_{\max }+S_{\min }}{2}
$$

As can be seen from Figure 2-1(b), the range of stress, $S_{r}$, is the algebraic difference 
between the maximum and minimum stress in a cycle.

$$
S_{r}=S_{\max }-S_{\min }
$$

The alternating stress can be obtained as follows:

$$
S_{a}=\frac{S_{r}}{2}=\frac{S_{\max }-S_{\min }}{2}
$$

\subsection{The Stress-Life $(S-N)$ Curve}

The basic method of presenting engineering fatigue data is by means of the $S-N$ curve, a plot of stress $S$ against the number of cycles to failure $N$. A log scale is almost always used for $N$. The value of stress that is plotted can be $S_{a}, S_{\max }$, or $S_{\min }$. The $S-N$ relationship is determined for a specific value of $S_{\mathrm{m}}$, stress ratio $R$, or amplitude ratio $A$ $[15,16]$.

Stress ratio: $\quad R=\frac{S_{\min }}{S_{\max }}$

Amplitude ratio: $\quad A=\frac{S_{a}}{S_{m}}=\frac{1-R}{1+R}$

Most determinations of the fatigue properties of materials are made in completely reversed bending where the mean stress is zero. Figure 2-2 gives the $S$ - $N$ curves for mild-steel and aluminum alloy obtained from rotating-beam tests $[5,11]$.

The $S-N$ approach is applicable to situations where cyclic loading is essentially elastic, meaning that the $S-N$ curve should be used in regions where lives greater than $100,000\left(N>10^{5}\right.$ cycles). This is because the $S-N$ curve is essentially flat in the low cycle 
region, and would yield an inaccurate estimation of life. Under these conditions the stress is elastic. At higher stresses the fatigue life is progressively decreased because the high load levels result the large plastic strain. For the low-cycle fatigue region $\left(N<10^{5}\right.$ cycles) tests are conducted with controlled strains of specimen instead of controlled stress cycles. Low cycle fatigue analysis is best treated by strain-based procedures that account for the effects of plasticity $[5,12,14]$.

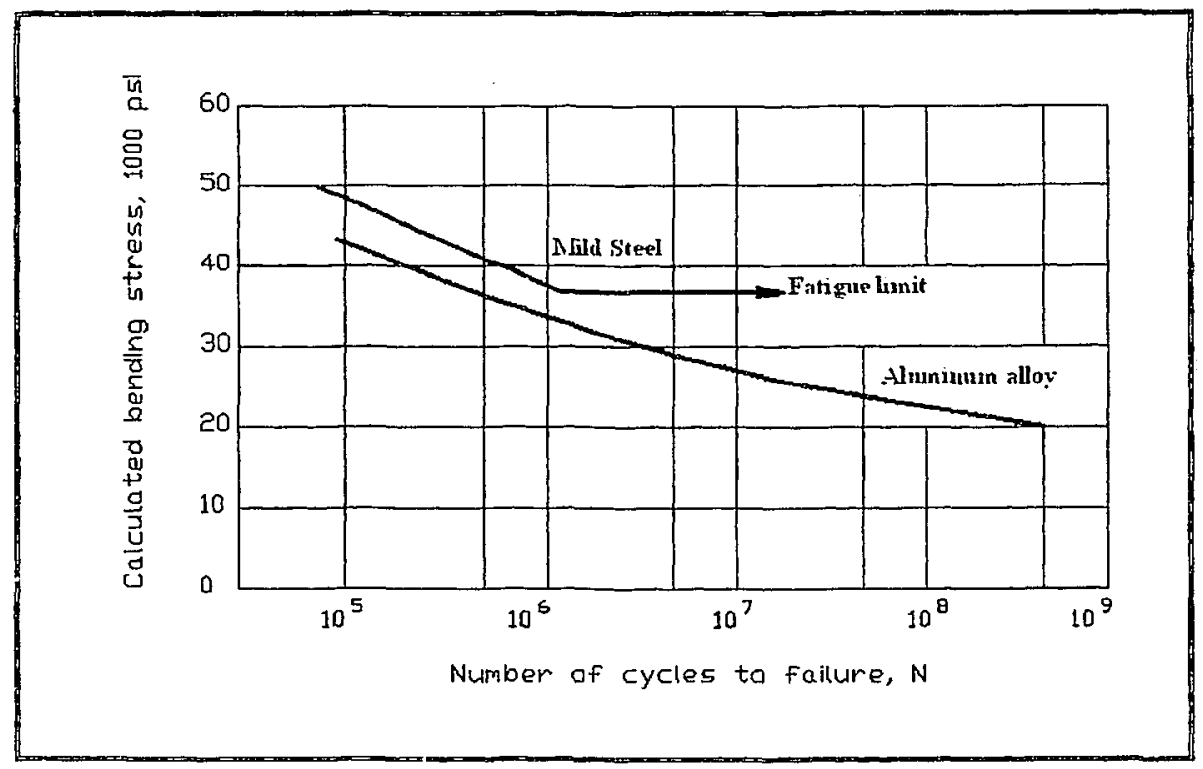

Figure 2-2 Typical $S-N$ Curves for Ferrous and Non-Ferrous Metals [5,11].

As can be seen from Figure 2-2, the number of cycles of stress that a metal can endure before failure increases with decreasing stress. Unless otherwise indicated, $N$ is taken as the number of cycles of stress to cause complete failure of the specimen. Fatigue tests at low stresses are usually carried out for $10^{7}$ cycles and sometimes to $5 \times 10^{8}$ cycles for nonferrous metal. For a few important engineering materials, such as steel, the $S-N$ curves become horizontal at a certain limiting stress. Below this limiting stress, which is called the fatigue limit or endurance limit, the material presumably can endure an infinite number of 
cycles without failure. Most non-ferror rals, like aluminum, have a $S-N$ curve which slopes gradually downward with increas $\iota_{-}$; , umber of cycles. These materials do not have a true fatigue limit because the $S-N$ curve never becomes horizontal $[5,17]$.

\subsection{Effect of Mean Stress on Fatigue}

Most of the fatigue data in the literature are determined for conditions of completely reversed cycles of stress, $S_{m}=0$. However, conditions are frequently met in engineering practice where the stress situation consists of an alternating stress and superimposed mean or steady stress $[16,17,18]$.

It is very important to fully characterize the effect of mean stress on the fatigue process so that laboratory data can be efficiently emplnyed in determining life calculations. Several empirical relations that characterize the effect of mean stress on fatigue life have been developed. The two relations have been most widely accepted are Goodman and Gerber $[19,20]$.

According to Goodman [19], the effect of mean stress is to linearly reduce the applied stress amplitude for both tensile and compressive mean stresses. This relationship can be summarized as follows:

$$
S_{a}=S_{o}\left[1-\frac{S_{m}}{S_{u}}\right]
$$

where, $S_{a}$ is the allowable stress amplitude, $S_{o}$ is the allowable stress amplitude at zero mean stress, $S_{m}$ is the mean stress and $S_{\mathfrak{u}}$ is the ultimate tensile stress.

Gerber, on the other hand, hypothesizes that the mean stress reduces the applied 
stress amplitude quadratically for both tensile and compressive state of mean stresses $[19,20]$. Gerber's relation can be summarized as:

$$
S_{a}=S_{0}\left[1-\left(\frac{S_{m}}{S_{u}}\right)^{2}\right]
$$

Experience has shown that the actual data fall between Goodman's and Gerber's relationships $[19,20]$. Unfortunately, little or no experimental data exists to support one approach over the other. It is therefore recommended that the approach yielding the most conservative life be selected.

\subsection{The Strain-Life ( $\varepsilon-N)$ Curve}

The strain versus fatigue life behaviour is commonly characterized by the strain-life $(\varepsilon-N)$ curve. Unlike the $S-N$ curve, the $\varepsilon-N$ curve is composed of elastic and plastic strain components. If the elastic and plastic strains are plotted separately against the number of reversals to failure on a log-log scale, a strain-life curve can be generated as shown in Figure 2-3 $[5,14,15]$. Therefore, the strain-life curve represents the total strain against reversals to failure, total strain being the arithmetic sum of elastic and plastic strains $[12,13,14]$.

The $S-N$ approach has been used extensively to investigate the premature failure of structures or components subjected to fluctuating loads [5]. Traditionally, the magnitude of

the stresses was low. Hence, the life cycles to failure are greater than $10^{5}$ cycles $[5,15,17]$. 


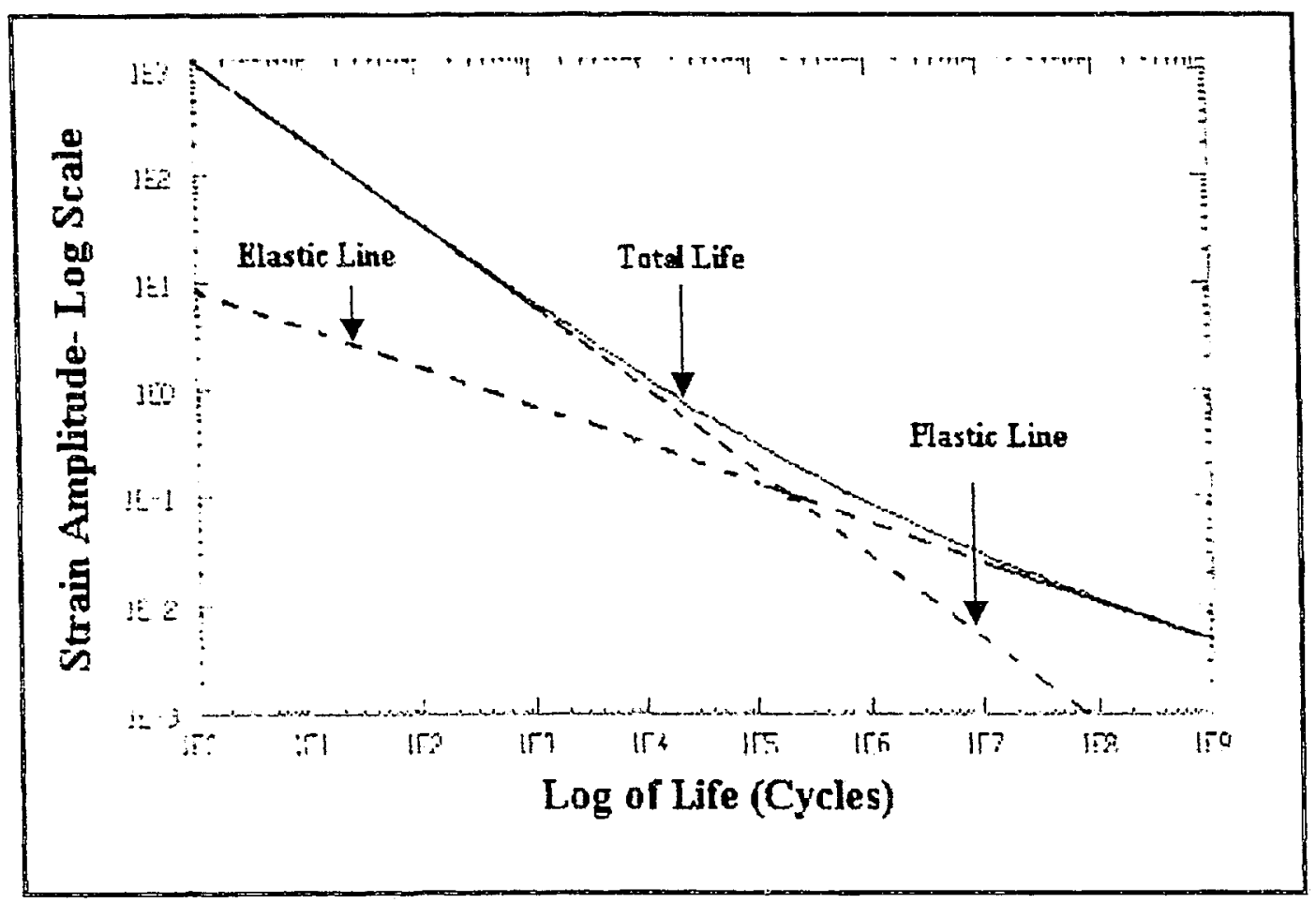

Figure 2-3 Typical Strain-Life $(\varepsilon-N)$ Curve [5].

This type of behaviour, as previously mentioned, is strictly confined within the elastic region of the material, before the yield point. However, as duty cycles have become more severe and the components more complicated, another pattern of fatigue behaviour emerges. In this regime, the cyclic stresses are very high, and a significant amount of plastic deformation is associated with the component $[5,17]$. As a result, the component has short lives in the range of $10^{2}$ to $10^{5}$ cycles. This type of behaviour is commonly referred to as low cycle fatigue or strain controlled fatigue. Unlike the nominal stress approach, the strain-life methodology is characterized by two curves, namely the strain-life curve and the cyclic stress-strain curve [5]. 


\subsection{Cyclic Stress-Strain Curve}

Cyclic strain controlled fatigue, as opposed to cyclic stress controlled fatigue, occurs when the strain amplitude is held constant during cycling. Strain controlled cyclic loading is found in thermal cycling, where a component expands and contracts in response to fluctuations in the operating temperature, or in reversed bending between fixed displacements $[18,19]$. Generally, the localized plastic strains at a notch subjected to either cyclic stress or strain conditions result in strain controlled conditions near the roof of the notch due to the constraint effect of the larger surrounding mass of essentially elastically deformed material $[20,21,22]$.

It is important to comprehend the behavior of a material when it is loaded monotonically. Tension tests on certain materials provide basic design data such as yield strength, ultimate tensile strength and ductility. In fatigue analyses, they provide baseline stress-strain curves for evaluating the nature and extent of any subsequently cyclically induced changes in deformation resistance (such as cyclic hardening or softening) [21,22].

In a tension test, a smooth cylindrical specimen is continually loaded with a tensile uni-axial load. The elongation of the specimen is simultaneously monitored throughout this process until the specimen fails. Figure $2-4$ shows a typical monotonic stress-strain curve $[5,14,15,21]$. 


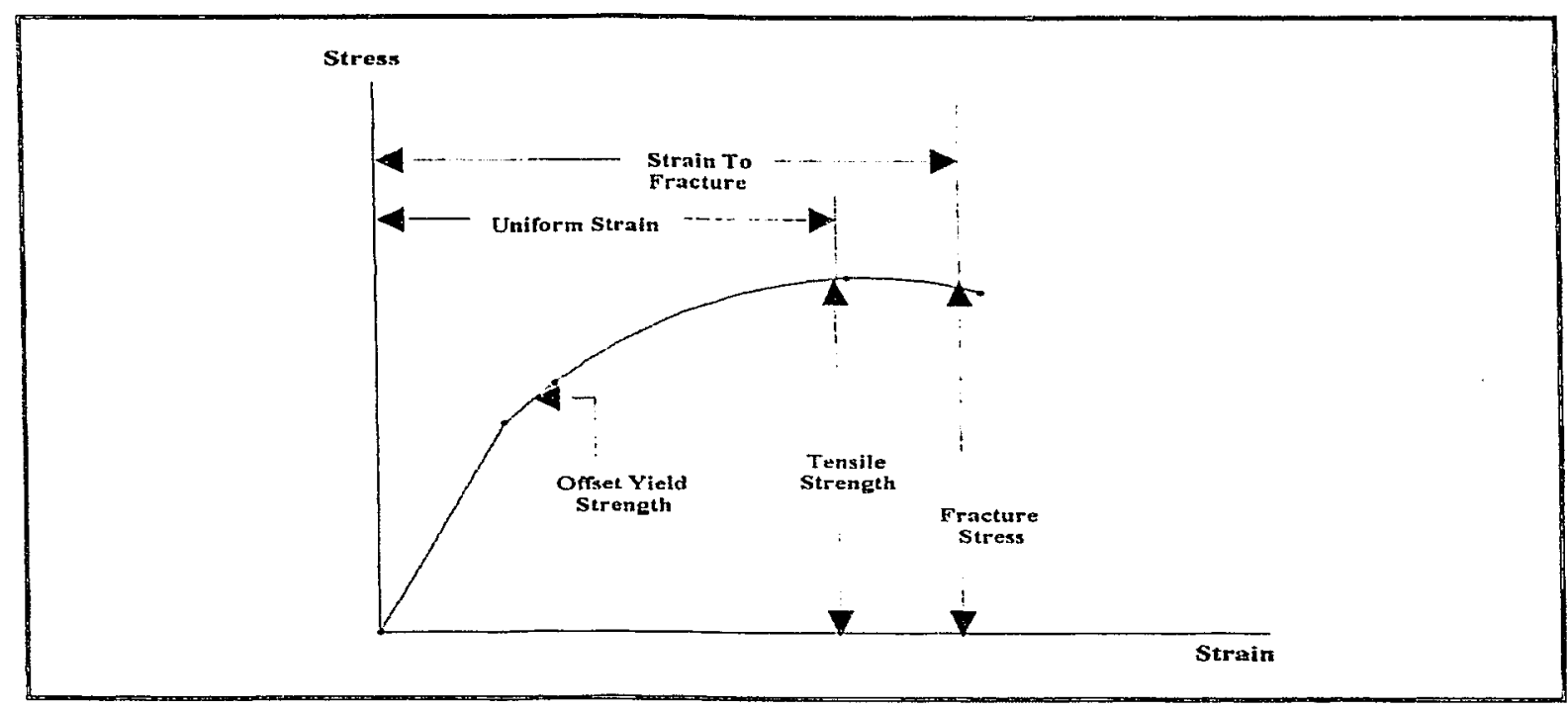

Figure 2-4 Typical Stress-Strain Curve [5].

The stress in the engineering stress-strain curve is calculated by subsequently dividing the applied load by the original cross-sectional area of the specimen. However, in reality, a true stress-strain curve yields the true behaviour of the material when loaded monotonically [23]. Figure 2-5 shows a true stress-strain curve as compared to its engineering counterpart.

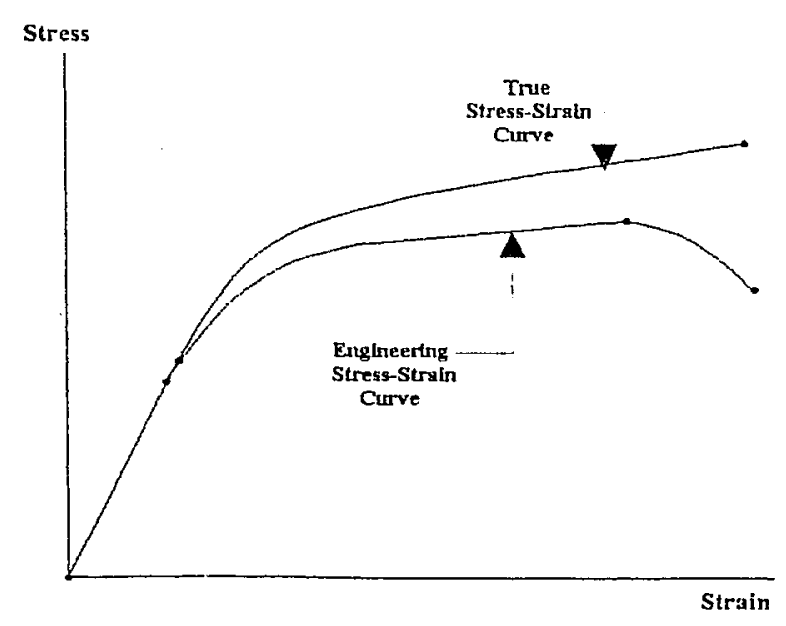

Figure 2-5 A Comparison between True and Engineering Stress-Strain Curves [23]. 
If during the course of a monotonic tension test the material is loaded to point B (Figure 2-6) and the load removed, the curve will follow a straight line denoted by line BC. The slope of $\mathrm{BC}$ is equivalent to the elastic modulus represented by OA. Indeed, the calculation of slope $\mathrm{BC}$ is the most accurate way of measuring the elastic modulus of the material. $[5,23]$

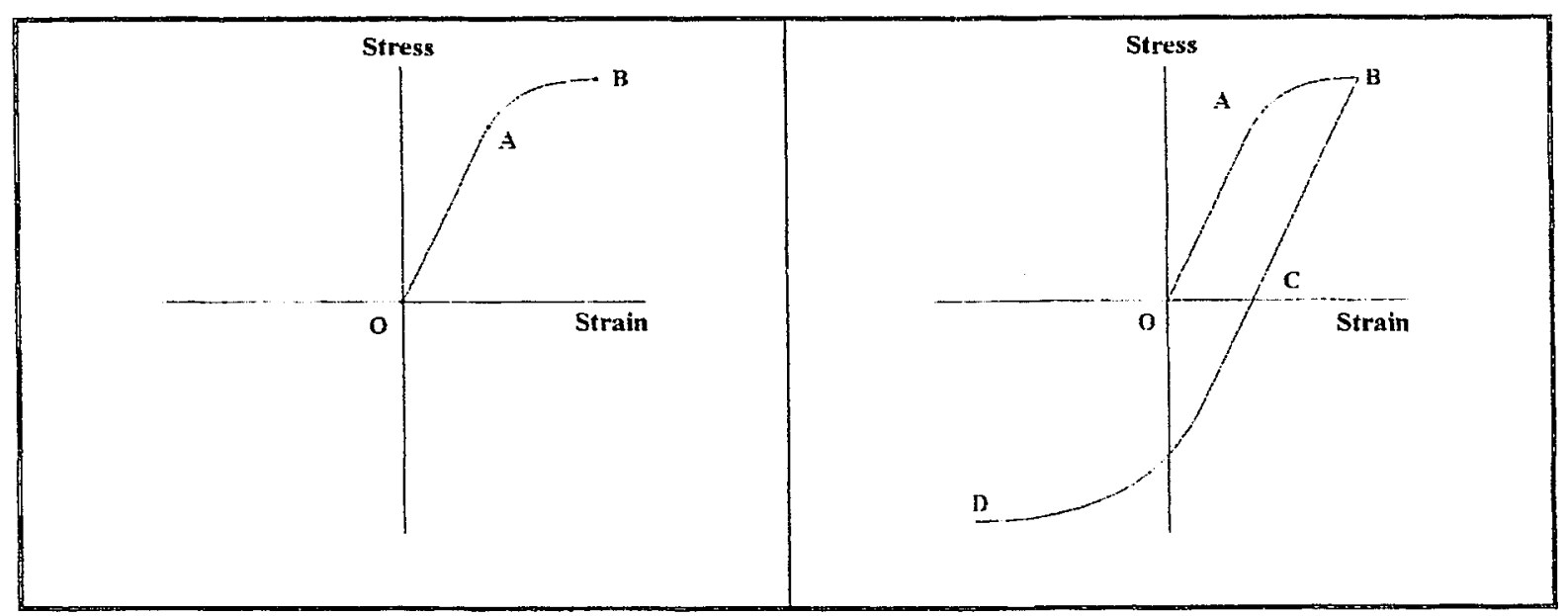

Figure 2-6 Loading Reversed into Compression [23].

Line $\mathrm{OC}$ represents the zero stress line, positive stress being tensile loading and negative, compressive loading. If the loading process $O B$ is reversed at point $C$, the material will be in compression up to point $D$. It should be noted that point $O$ is equidistant from points $B$ and $D$, both horizontally and vertically. At point $D$, the process $O B$ is repeated, both in magnitude and direction. The curve will then follow the trend DB, forming a complete stress-strain cycle also known as a hysteresis loop (Figure 2-7).

The above process is the first step towards a cyclic stress-strain curve. If the material is continuously cycled between fixed strain limits, one of the following four events may happen. 


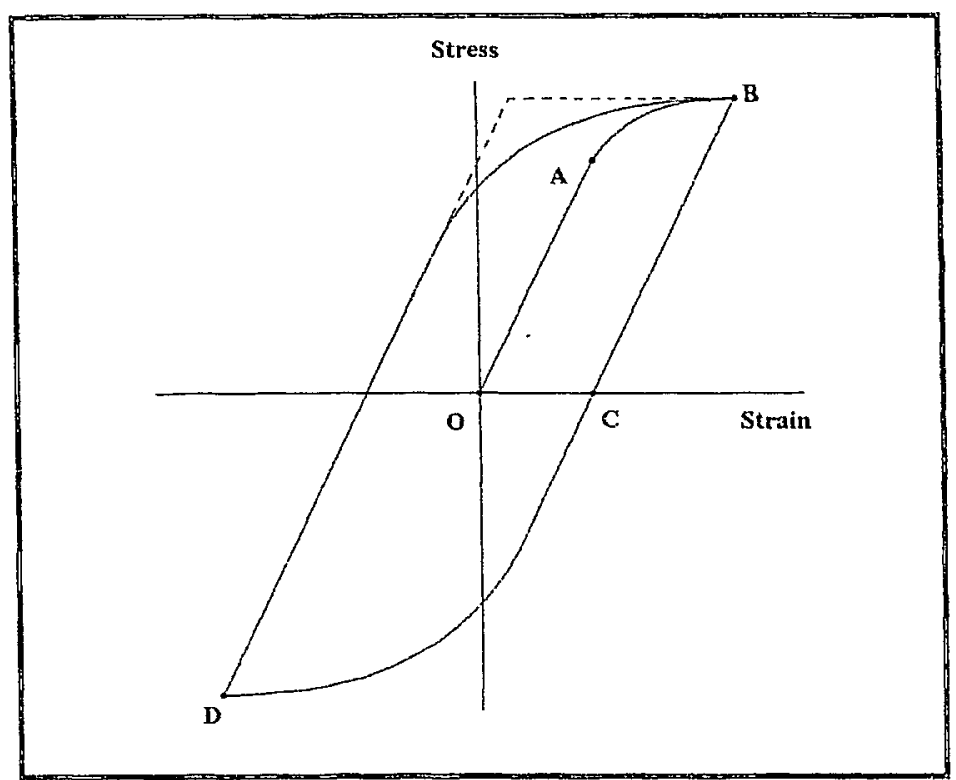

Figure 2-7 A Complete Stress-Strain Cycle, a Hysteresis Loop [23].

Depending on the properties of the material and the heat treatment, the material may $[5,23,24]$ :

- cyclically soften

- cyclically harden

- remain stable

- cyclically harden and soften depending on strain range (mixed behaviour)

The onset of cyclic softening and hardening is illustrated in Figure 2-8 where two different materials are tested under fixed strain limits. Although the strain is fixed, the loads vary significantly in that they converge to different levels.

The discrepancies in load levels demonstrate the material's response to the cyclically induced strain, thus causing the softening or hardening behaviour. It is apparent from Figure 2-8 that the maximum stress decreases with number of imposed cycles in the 
case of softening whereby the stress increases during the course of hardening $[5,23,24,25]$. In both processes, the stresses converge to a certain limit and remain stable until the emergence of a fatigue crack. The hardening and softening behaviour of materials can be mathematically expressed in terms of the ratio of ultimate tensile strength and $0.2 \%$ proof strength as follows [5]:

$$
\begin{aligned}
& \frac{S_{u}}{S_{0.2}}>1.4 \\
& S_{u-}<1.2 \\
& S_{0.2}
\end{aligned}
$$

For ratios greater than 1.4 , the material cyclically hardens, whereas a ratio less than 1.2 implies a softening behavior. Within the range of 1.2 to 1.4 , the material can cyclically soften, harden or exhibit a mixed behavior [5].
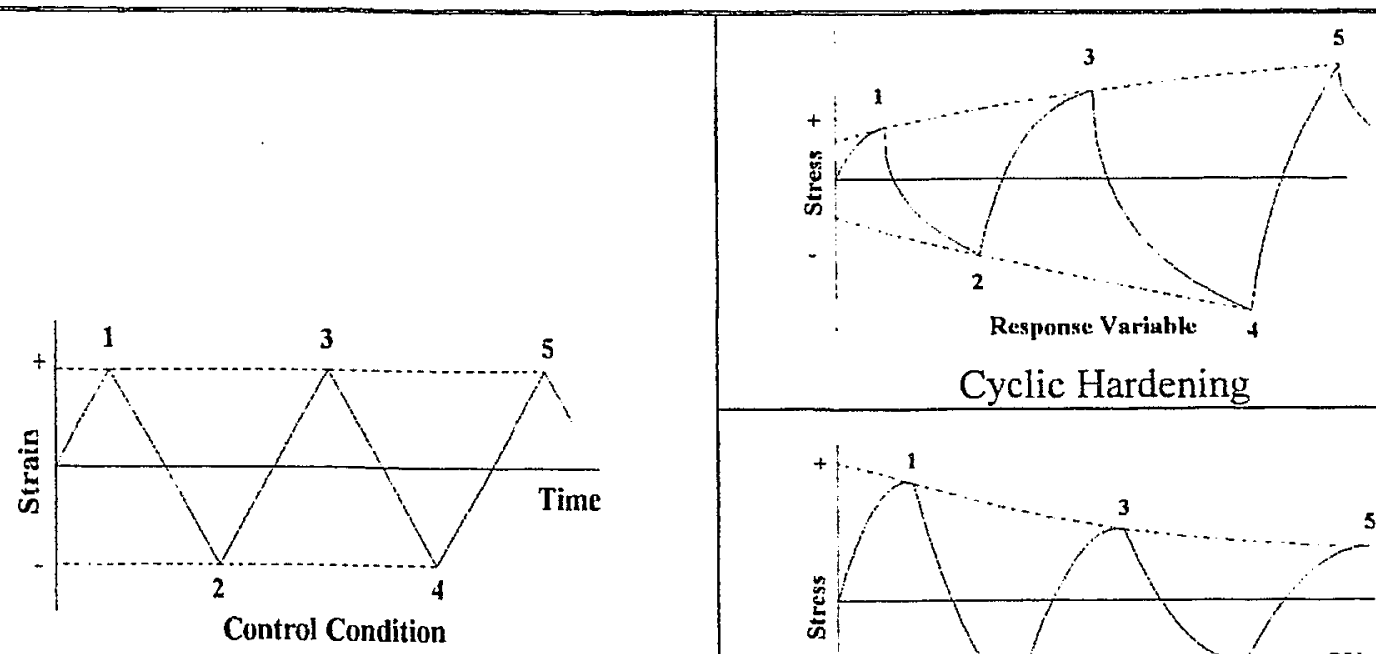

Cyclic Hardening

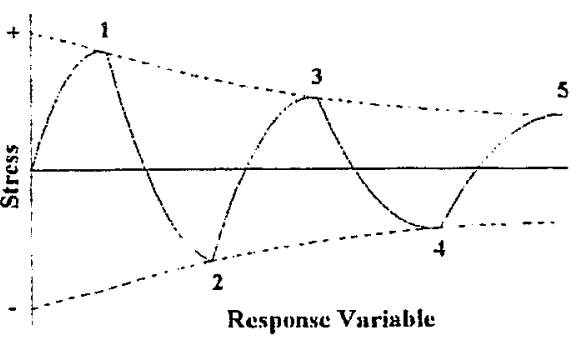

Control Between Fixed Strain Limits

Cyclic Softening

Figure 2-8 Cyclic Softening and Hardening under Strain Control $[5,23,24]$. 
After a relatively small number of cycles no more than about $10 \%$ of the material's total life the hysteresis loop tends to stabilize, meaning the stress amplitude remains constant over the entire life portion $[5,24,25]$. If the stress-strain coordinates relating to the tips of the hysteresis loops are plotted, the locus of these points generates the cyclic stressstrain curve as shown in Figure 2-9.

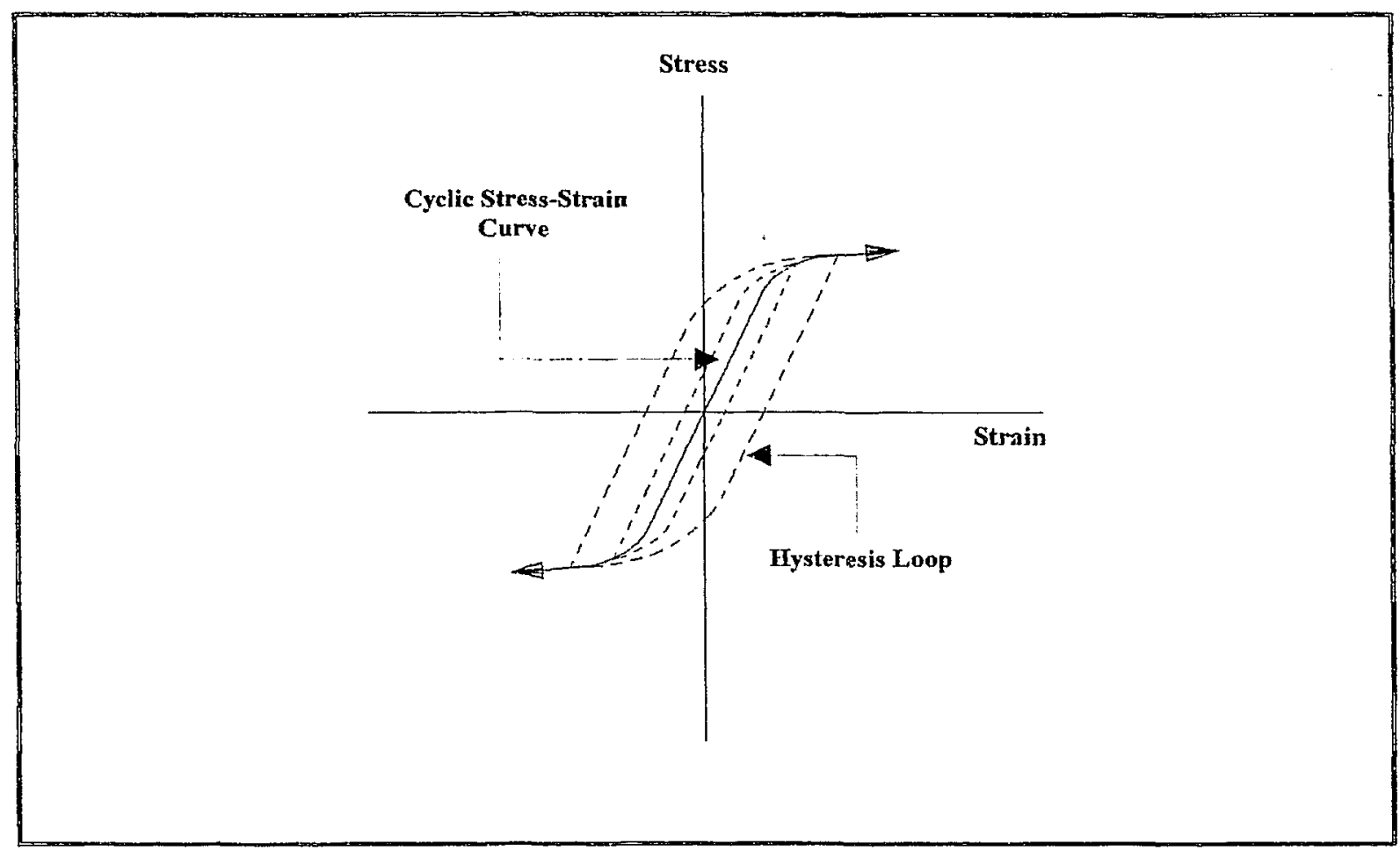

Figure 2-9 The Cyclic Stress-Strain Curve [5].

Unlike the true stress-strain curve, the cyclic stress-strain curve defines the material's behaviour under cyclic loading conditions. The cyclic stress-strain curve can be directly compared to its monotonic counterpart to quantitatively assess the softening and hardening behaviour of the material. When a material cyclically softens, the cyclic yield strength is lower than the monotonic yield strength whereas in the case of hardening, the 
monotonic yield strength is higher than that of the cyclic loading. Figures 2-10 (a) through (d) show a comparison between cyclic and monotonic behaviour for mild-steel $[5,23,25]$.

Although the cyclic stress-strain curve is generated experimentally, it can also be mathematically expressed as follows $[5,23,24,25]$ :

$$
\varepsilon_{t}=\frac{\sigma}{E}+\left(\frac{\sigma}{K^{1}}\right)^{\frac{1}{n^{1}}}
$$

where, $\varepsilon_{t}$ is the total strain, $\sigma$ is the stress amplitude, $E$ is the elastic modulus, $K^{1}$ is the cyclic strength coefficient and $n^{1}$ is the cyclic strain hardening exponent.

For a known value of $E$ and coefficients $K^{1}$ and $n^{1}$, the cyclic stress-strain curve can be plotted for any material through the above equation.

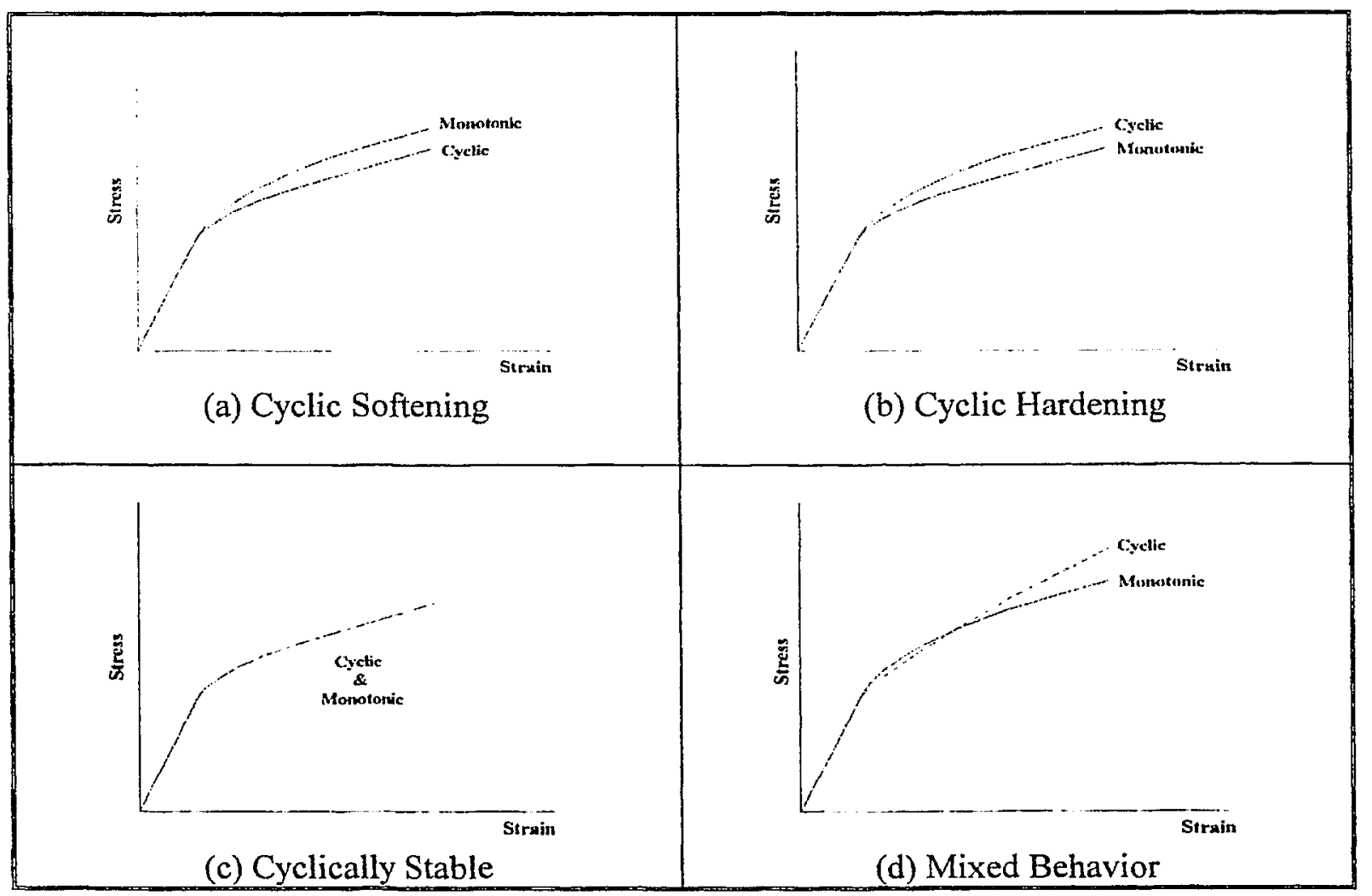

Figure 2-10 Comparison between Cyclic and Monotonic Stress-Strain Curve [25]. 


\subsection{Design for Fatigue}

Considerable literature has been developed on methods and procedures for designing against fatigue. The most appropriate references are given in references [24-26]. There are several distinct philosophies concerning design for fatigue that must be understood to put this vast subject into proper perspective.

1- Infinite-life design. This design criterion is based on keeping the stresses at some fraction of the fatigue limit of steel. This is the oldest fatigue design philosophy. It has largely been supplanted by other criteria discussed below. However, for situations in which the part is subjected to very large cycles of uniform stress it is valid design criterion.

2- Safe-life design. Safe-life design is based on the assumption that the part is initially flaw-free and has a finite life in which to develop a critical crack. In this approach the designer must observe that fatigue life at a constant stress is subjected to large amounts of statistical scatter [25]. For example, the Air force historically designed aircraft to a safe life that was one-fourth of the life demonstrated in full-scale fatigue tests of production aircraft. The factor of four was used to account for environmental effects, material property variations, and variations in manufacturing quality. Bearings are another good example of parts that are designed to a safe-life criterion. For example, the bearing may be rated by specifying the load that $90 \%$ of all bearings are expected to withstand over a given lifetime [26]. Safe-life design also is common in pressure vessel, jet engine, and fuel tank design.

3- Fail-safe design. In fail-safe design the view is that fatigue cracks must not lead to 
failure before they can be detected and repaired. This design philosophy was developed in the aircraft industry, where the weight penalty of using large safety factors could not be tolerated but neither could the danger to life from very small safety factors. Fail-safe designs employ multiple-load paths and crack stoppers built into the structure along with rigid regulations and criteria for inspection and detection of cracks [26].

4- Damage-tolerant design. The latest design philosophy is an extension of the failsafe design philosophy. In damage-tolerant design, the assumption is that fatigue cracks exists in an engineering structure. The techniques of fracture mechanics are used to determine whether the cracks grow large enough to cause failure before they are sure to be detected during a periodic inspection. The emphasis in this design approach is on using materials with high fracture toughness and slow crack growth $[25,26]$. The success of the design approach depends upon having a reliable nondestructive evaluation program and in being able to identify the critical damage areas in the design.

Although much progress has been made in designing for fatigue, especially through the merger of fracture mechanics and fatigue, the interaction of many variables that are typical of real fatigue situations makes it inadvisable to depend on a design based solely on analysis. Simulated service testing should be part of all critical fatigue applications [15]. The failure areas that are not recognized in design are detected by these tests. Simulating the actual service loads requires great skill and experience. Often it is necessary to accelerate the test, but doing so may produce misleading results. For 
example, when time is compressed in that way, the full influence of corrosion or fretting is not measured, or the overload stress may appreciably alter the residual stresses. It is common practice to eliminate many small load cycles from the load spectrum, but they may have an important influence on fatigue crack propagation [24].

\subsection{Application of Finite Element Method}

In automotive industries, fatigue life is estimated by using a numerical technique including both a discretized model of the structure and an associated load-time history that represents a specified event in the life of the structure. To facilitate this technique, FEM can be used. FEM is certainly a great advantage when the fatigue life of complex geometries is being estimated. In this chapter, the traditional and advanced fatigue mechanisms were discussed. Whether it is the $S-N$ or $\varepsilon-N$ approach, high-cycles or lowcycles fatigue, they all require obtaining stresses and strains. The next chapter discusses another type of application of FEM in flow analysis. 


\section{CHAPTER-3 SLOSHING SIMULATION}

In this chapter, FEM is used to model a fuel tank structure and liquid inside. Some specified techniques in Computational Fluid Dynamics (CFD) are employed to simulate sloshing in an excited tank and determine the liquid impact loads exerted on the tank walls.

\subsection{Introduction}

Modeling of the sloshing behaviour of liquids in externally excited containers has been studied in several applications, e.g., fuel slosh in aerospace applications, vehicle and ship dynamics, and earthquake engineering [3]. As mentioned previously, liquid sloshing in

a moving container constitutes a broad class of problems related to the safety of transportation systems, such as tank trucks on highways, and car fuel tanks on roads, and it is considered as one of the most important subjects in design of tank $[3,4,7]$. This phenomenon has attracted the attention of numerous researchers in the area of CFD.

When a tank is partially filled with liquid, a free surface is presented. Then, rigid body acceleration of the tank produces a subsequent sloshing of the fluid, and motion of the free surface, which can be studied based on a linear or a non-linear approach [7-10]. The elevation of the free surface or the amplitude of slosh depends on the amplitude and frequency of the tank motion, the liquid fill depth, liquid properties, and tank geometry. When the frequency of the tank motion is close to one of the natural frequencies of the tank's fluid, large sloshing amplitudes can be expected $[27,28]$.

A liquid's motion inside its container has an infinite number of natural frequencies, 
but it is the lowest few modes that are most likely to be excited by the motion of a vehicle. Most studies therefore have concentrated on investigating forced harmonic oscillations near the lowest natural frequencies, predicted by the linear fluid equations. However, non-linear effects result in the frequency of maximum response being slightly different from the natural frequency of tank and depend on amplitude of slosh [7].

The first and most complete study of the sloshing problem based on a linear theoretical approach and experiments were conducted by Abramson [27] for space vehicle applications. In his work, it is shown that in some cases the linearized theory of slosh may correctly predict the liquid behaviour in a harmonically excited container. The linear theoretical and experimental results agree well if the amplitude of excitation is small enough that the first natural frequency of the liquid motion is equal to the natural frequency of tank. For this reason, much of the sloshing technology developed for space industries is not applicable to road containers.

Liquid sloshing in moving tankers can lead to lateral and roll instabilities, decreased controllability/maneuverability, and increased stress on tank structures. The influence of large amplitude liquid sloshing on the overturning and skidding stability of road tankers is very serious during dynamic maneuvers [8]. An impulsive acceleration to a fuel road container can result in impact hydrodynamic pressure or impact loads of the liquid free surface on the tank walls. The non-linearity is mainly due to extremely rapid velocity changes during impacts. These changes are usually treated as being instantaneous (valocity jumps) and they lead to various strongly nonlinear features of system behavior. Even if the system is linear with constant coefficients and is subjected to impact loading, it will 
experience nonlinear behavior [8,9]. Most of the non-linear liquid sloshing studies have been reported recently in $[8,9,29,30]$. However, analytical techniques for predicting large amplitude sloshing are still not fully developed. Such loads are extremely important in the design stage of the supporting structure and internal components of vehicle tanks.

The most important task in the analysis of the flow in a moving container is the determination of the location of the moving free surface and hydrodynamic pressures. The location and transport of the free surface in the tank was addressed using a numerical method known as the volume of fluid (VOF) method, which was first introduced by Hirt and Nichols in 1981 [16]. The VOF method is a powerful method based on a function $F$ (volume fraction of fluid) whose value is unity at any point occupied by fluid and zero elsewhere. The flow field is discretized into many small cells and then the equations of motion are satisfied in each cell. The flexibility of this method suggests that it can be applied to the numerical simulation of sloshing [16] and is therefore used as a base in this study.

\section{2 Volume Of Fluid Method and Finite Element}

FE and VOF methods are employed to discretize the flow domain into small cells (elements) and simulate fuel sloshing to obtain the pressure distributions as well as the free surface locations as functions of time in a moving fuel tank. In the VOF method, Equation (3-1) for the volume fraction $F$ is solved simultaneously with other governing equations, i.e., the continuity and momentum conservation equations. 


$$
\frac{\partial F}{\partial t}+u . \nabla F=0
$$

where $F(x, y, t)=\frac{\text { Volume of Fluid }}{\text { Volume of Element }}$, and $u$ is the flow velocity vector.

Depending on the value of the volume fraction $F$ in a cell, the whole domain is divided into three categories by the following criterion:

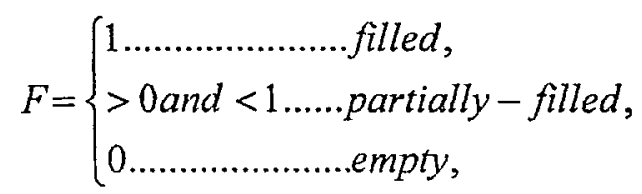

$F$ is unity in the fluid region and zero in the region where the fluid is absent. Therefore, the free surface can be considered to exist in the partially filled elements with $F$ values between zero and unity. In order to locate the free surface precisely, the number of cells involved in this region should be kept as small as enough [28]. Figure 3-1 shows a typical pattern of the free surface when the fluid sloshes in a tank.

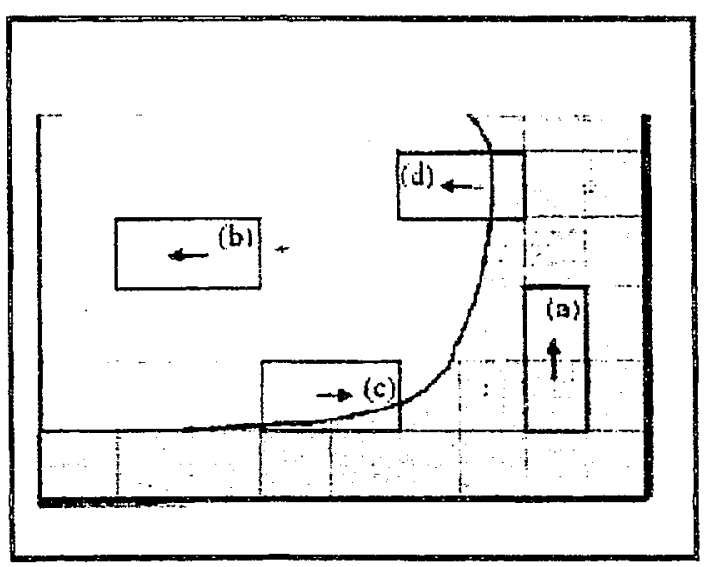

Figure 3-1 Typical Pattern of the Free Surface of Flow in a Discretized Tank [28].

Equation (3-1), in divergence form, is convenient for numerical approximation, because changes in $F$ in a cell reduce to fluxes of $F$ across the cell faces. Since $F$ is a step 
function, fluxes must be computed carefully to avoid smearing of the discontinuities [7]. The donor-acceptor technique, developed by Hirt and Nichols, is a popular technique for overcoming such smearing of the free surface [16]. When the cell is near the interface between two phases, a donor-acceptor technique is used to determine the amount of fluid advected through the face. This technique identifies one cell as a donor of an amount of fluid from one phase and another (neighbor) cell as the acceptor of the same amount of fluid, and is used to prevent numerical diffusion at the interface. The amount of fluid from one phase that can be convected across a cell boundary is limited by the minimum of two values: the filled volume in the donor cell or the free volume in the acceptor cell. Figure 3-2 shows a typical pattern of an actual free surface interface and the interface shape represented by donor-acceptor technique.

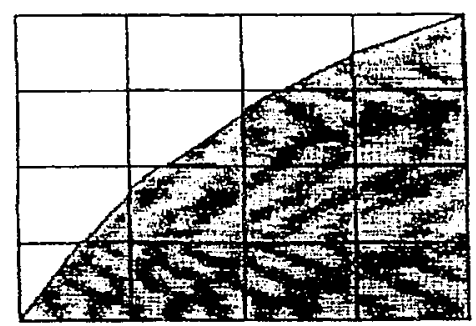

(a) Actual interface shape

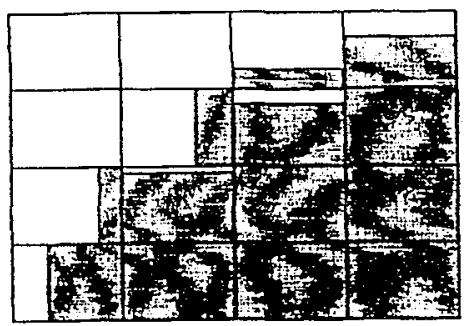

(b) Interface shape represented by the donor-acceptor technique

Figure 3-2 Typical Pattern of the actual Free Surface Interface and Donor-Acceptor Technique

The objective of simulating the liquid sloshing reported here, is to obtain the freesurface location and hydrodynamics pressure distributions of the fluid as functions of time. The governing equations, the continuity and momentum conservation equations, are based 
on Navier-Stockes equations [7,31] for Newtonian, incompressible, isotropic fluid.

\subsection{Finite Element Modeling of Fuel Tank}

The modeling process of the tank structure involves two steps, namely geometry modeling, and FE discretizing. First the geometry or computer aided design (CAD) model of fuel tank with dimensions $50 \times 50 \times 30 \mathrm{~cm}^{3}$, and three straight baffles installed on the bottom of the tank with eight (8) $\mathrm{cm}$ height were created in pre-processor package GAMBIT. This tank allows for 75 liters fuel that is close to light truck and passenger cars fuel tank. Fig.3-3 shows the schematic drawing of the tank with three baffles and Figure 34 shows the CAD model of the tank with coordinate system, which will be used for all acceleration and load directions.

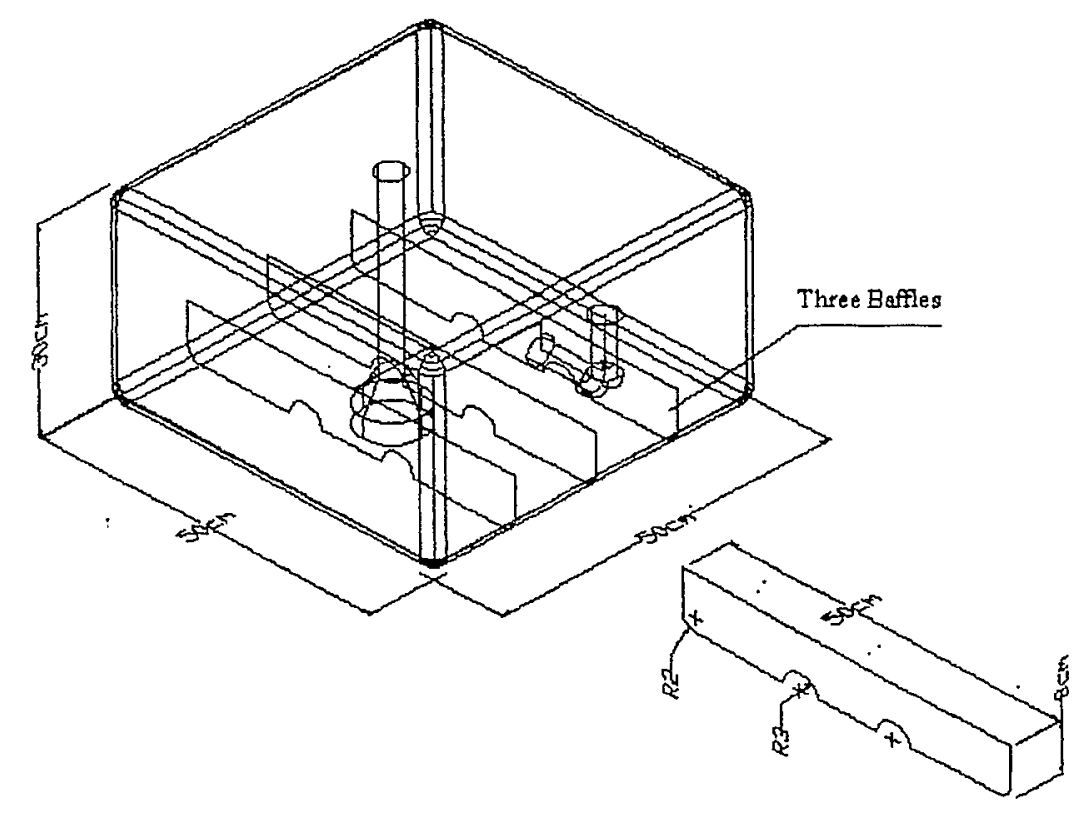

Figure 3-3 Schematic Drawing of the Fuel Tank with three Baffles. 


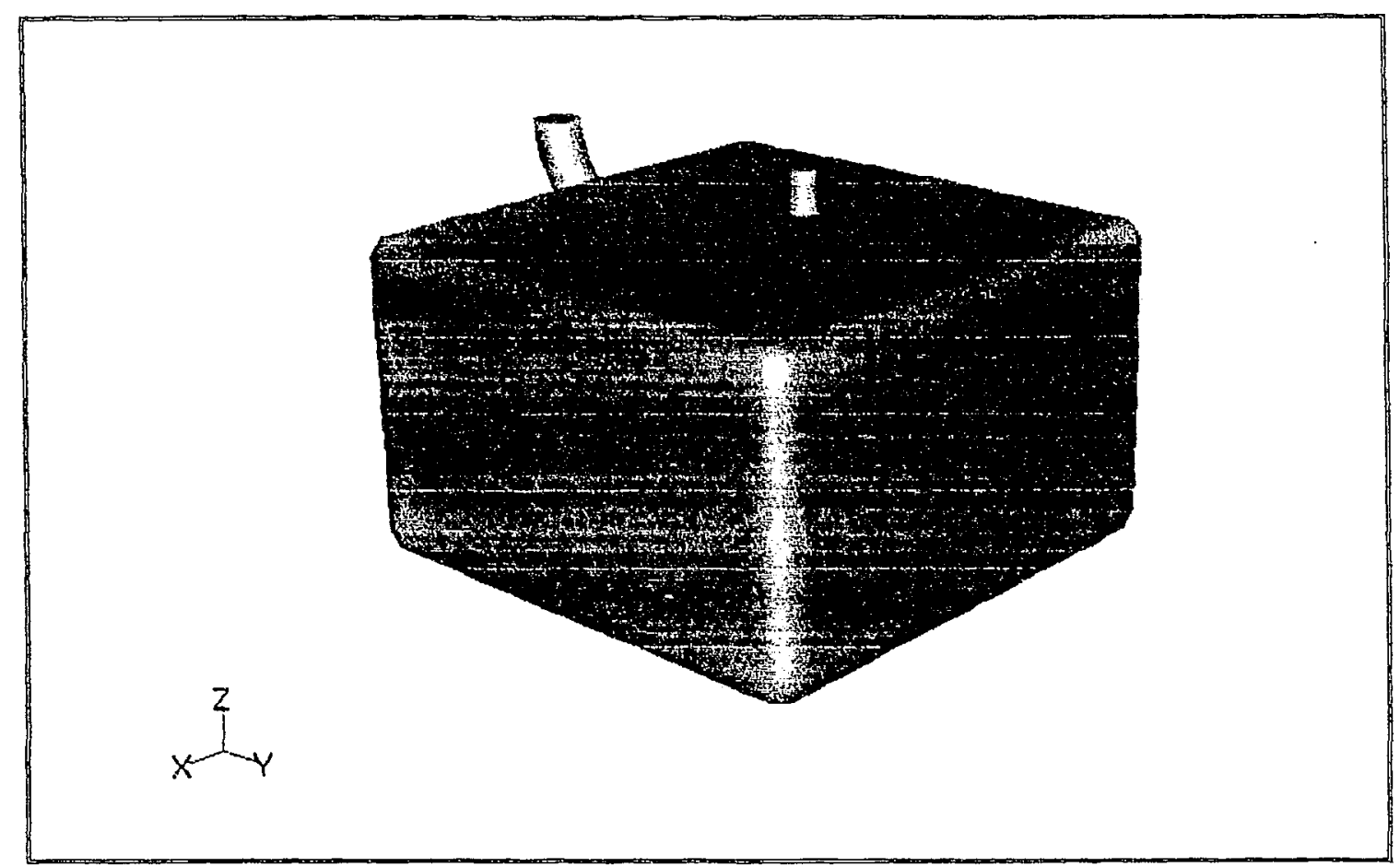

Figure 3-4 CAD Model of Fuel Tank.

After all surfaces were created in pre-processor, each part is separately discretized. The correct selection of elements would yield better results that are close to realistic solutions. Some of the elements are only applicable to certain situations and some work better than others in a particular situation. In CFD simulation, discretizing of the tank walls and baffles is for the purpose of making boundary on the flow domain. Therefore, 2D shell elements are quiet appropriate for meshing the tank walls and baffles as they can be extruded to three dimensional (3D) elements (cells) for discretizing of flow domain [32]. A combination of two dimensional (2D) elements, Quad4-nodes and Tria3-nodes, has been used to discretize the tank shell. The 2D elements are then extruded to create $3 \mathrm{D}$ elements. This procedure is done by extruding the $2 \mathrm{D}$ elements of the model in each direction normal 
to the surface of the element. As a result, Tria3 and Quad4 become Wedge- 6 and Hex-8, $3 \mathrm{D}$ elements respectively. The fluid flow domain inside the tank is discretized by a combination of tetrahedral and hexahedral elements. Fig 3-5 shows the model of the tank, which has been meshed in GAMBIT. Final model contains 90,772 3D-elements including combination of tetrahedral and hexahedral elements for fluids cells inside the tank and 8,892 2D-elements including combination of Tria3 and Quad4 for walis of the tank and baffles.

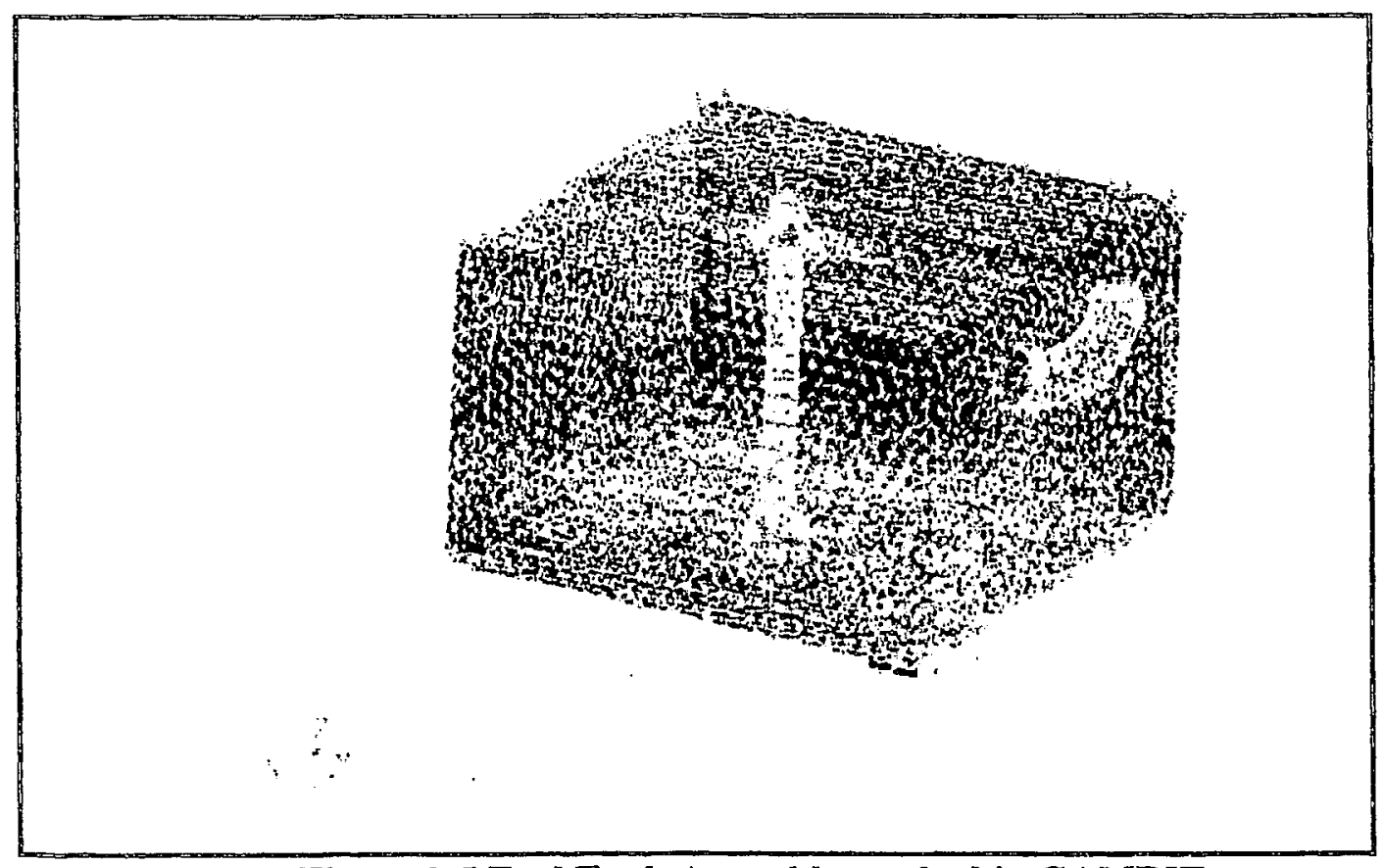

Figure 3-5 Fuel Tank Assembly meshed in GAMBIT.

\subsection{Sloshing Analysis}

\subsubsection{Introduction}

The sloshing simulation herein is based upon finite element analysis (FEA) using 
VOF method and the donor-acceptor technique. The commercial package, FLUENT is used to run the simulations. The Navier-Stokes equations are solved over a discrete domain made of small elements. These equations are integrated over each element, resulting in a set of nonlinear system of equations, which are solved iteratively to obtain the pressure, and the location of the fluid interface over the discrete domain. It has been assumed that there are two immiscible fluids, such as liquid fuel and air, interacting with each other during sloshing in a rectangular tank in an accelerating car.

After creating the meshed model of the tank the descritized model is imported to FLUENT to check the elements, apply boundary conditions, operating conditions, material properties, and then solving the model. Solution of the model under specific conditions is applied according to the following steps. Figure 3-6 shows the imported discretized model of the tank to FLUENT.

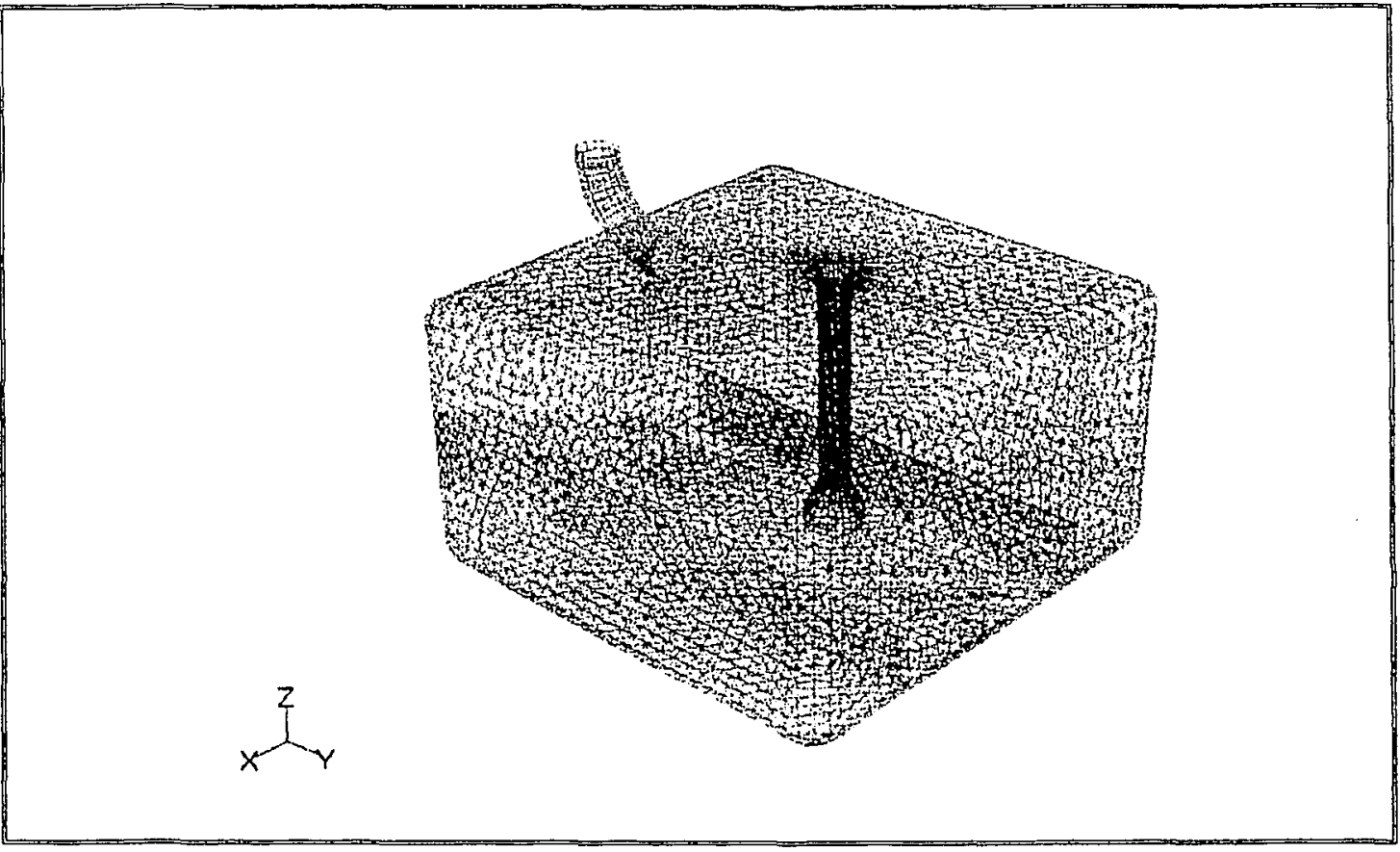

Figure 3-6 Imported Discretized Model of the Tank to FLUENT. 


\subsubsection{Overview of Numerical Algorithm}

Two numerical Algorithms can be used in FLUENT for solving the problem[34,35]:

1- Segregated Solution Algorithm (SSA)

2- Coupled Solution Algorithm (CSA)

Using either algorithm will solve the governing equations for the conservation of mass and momentum, and for energy and other scalars [32]. In both methods a VOF based technique is used that consists of:

- Division of domain into discrete control volumes using a computational grid.

- Integration of the governing equations on the individual control volumes to construct algebraic equations for the discrete dependent variables such as velocities, pressure, and conserved scalars.

- Linearization of the discretized mathematical model and solution of the resultant linear equation system to yield updated values of the dependent variables $[31,32]$.

The two numerical algorithms employ a similar discretization process, FE, but the approach used to linearize and solve the discretized equations is different.

\subsubsection{Segregated Solution Algorithm (SSA)}

Using the SSA, the governing equations are solved sequentially (i.e., segregated from one to another). Since the governing equations are non-linear, several iterations of the solution loop must be performed before a converged solution is obtained [31,32]. Each iteration, consists of the steps illustrated in Figure 3-7 and outlined below: 
1- Fluid properties are updated, based on the current solution.

2- The velocities in momentum equations are each solved in turn using current values for pressure and face mass fluxes, in order to update the velocity field.

3- Since the velocities obtained in Step 2 may not satisfy the continuity equation locally, a Poisson-type equation for the pressure correlation is derived from the continuity equation and the linearized momentum equations. This pressure correlation equation is then solved to obtain the necessary correlations to the pressure and velocity fields and the face mass fluxes such that continuity is satisfied.

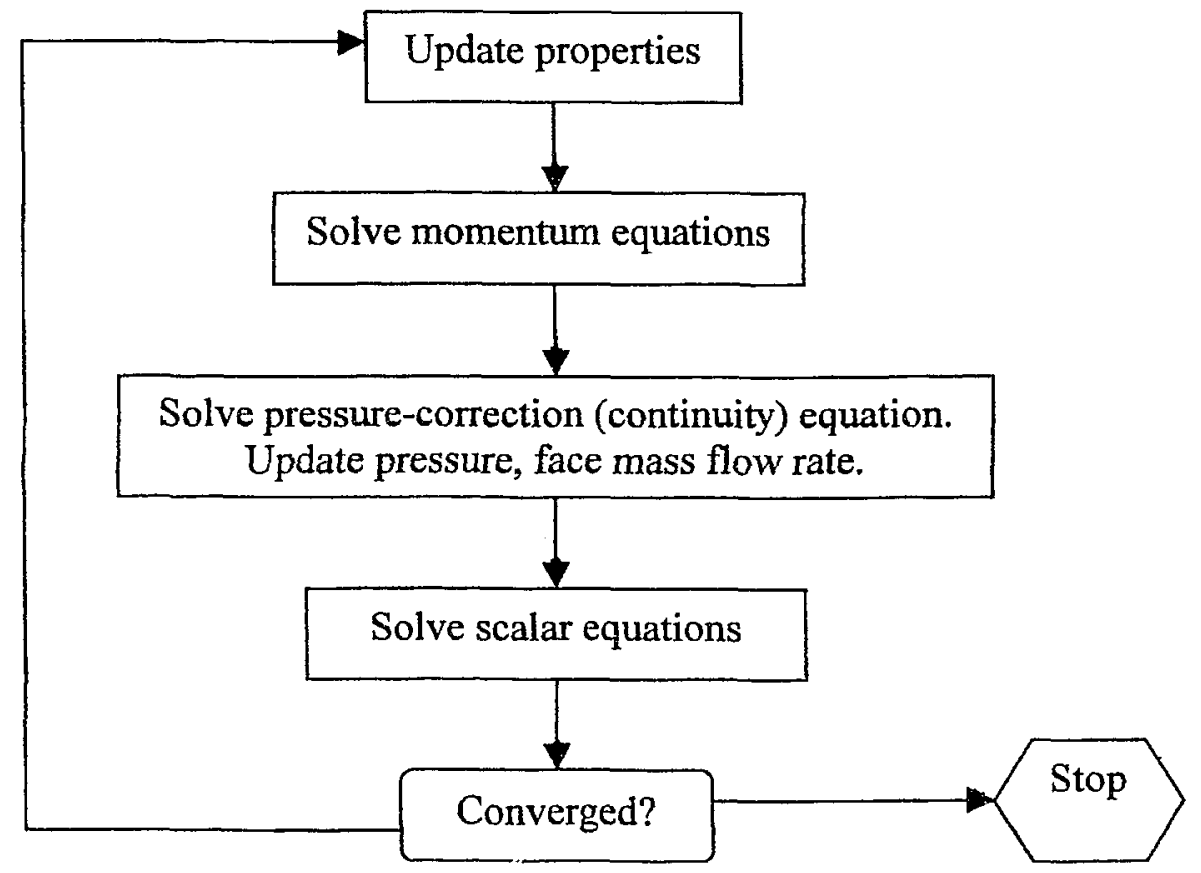

Figure 3-7 Overview of the Segregated Solution Algorithm (SSA) [32]. 
4- Equations for scalars are solved using the previously updated values of the other variables.

5- A check for convergence of the equation set is made.

These steps are continued until the convergence criteria are met.

\subsubsection{Coupled Solution Algorithm (CSA)}

The coupled solver solves the equations of continuity, momentum, energy, and species transport simultaneously. Governing equations for additional scalars will be solved sequentially. Because the governing equations are non-linear (and coupled), several iterations of the solution loop must be performed before a converged solution is obtained [35]. Each iterations consists of the steps illustrated in Figure 3-8 and outlined below:

1- Fluid properties are updated based on the current solution.

2. The continuity, momentum, energy, and species equations are solved simultaneously.

3- Equations for scalars are solved using the previously updated values of the other variables.

4- When inter-phase coupling is to be included, the source terms in the appropriate continuous phase equations may be updated with a discrete phase trajectory calculation.

5- A check for convergence of the equation set is made.

These steps are continued until the convergence criteria are met. 


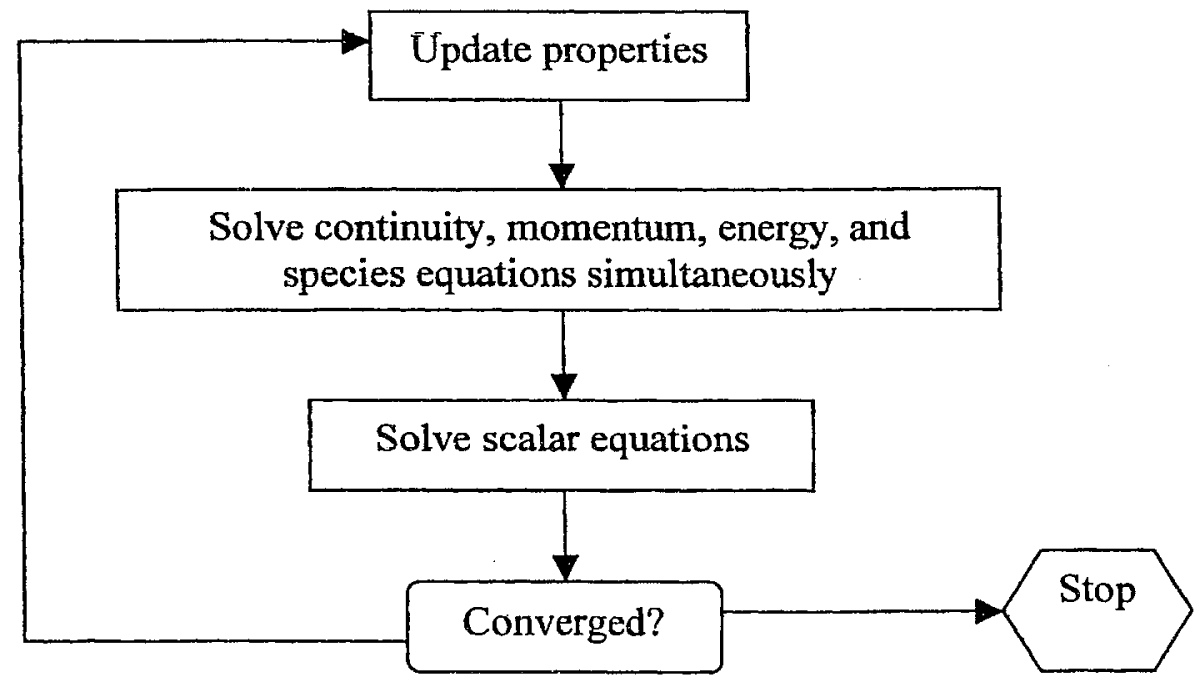

Figure 3-8 Overview of the Coupled Solution Algorithm (CSA) [32].

\subsubsection{Linearization: Implicit vs. Explicit}

In both the segregated and coupled solution algorithms the discrete, non-linear governing equations are linearized to produce a system of equations for the dependent variables in every computational cell. The resultant linear system is then solved to yield an updated flow field solution $[27,32]$.

The manner in which the governing equations are linearized may take an implicit or explicit form with respect to the dependent variable (or set of variables) of interest. By implicit or explicit means the following:

Implicit: For a given variable, the unknown value in each cell is computed using a relation that includes both existing and unknown values from neighboring cells. Therefore each unknown will appear in more than one 
equation in the system, and these equations must be solved simultaneously to give the unknown quantities [31-33].

Explicit: For a given variable, the unknown value in each cell is computed using a relation that includes only existing values. Therefore each unknown will appear in only one equation in the system and the equations for the unknown value in each cell can be used one at a time to give the unknown quantities [31-33].

In SSA each discrete governing equation is linearized implicitly with respect to that equation's dependent variable. This will result in a system of linear equations with one equation for each cell in the domain. Because there is only one equation per cell, this is sometimes called a scalar system of equations [32].

In summary, due to the description of the VOF and donor-acceptor technique, which has been described previously, it seems that the implicit SSA approach can be an appropriate method for simulating of slosh in moving tank. The segregated approach implicitly solves for a single field variable (e.g., hydrodynamic pressure) by considering all cells at the same time. It then solves for the next field variable by again considering all cells at the same time. When body force variables (e.g. gravity force $=9.81 \mathrm{~m} / \mathrm{s}^{2}$ ) exist in multiphase flows (here, air and fuel), the body force and pressure gradient terms in the momentum equation are almost in equilibrium, with the contributions of convective and viscous terms are small in comparison. The segregated solution algorithms converge poorly unless partial equilibrium of pressure gradient and body forces is taken into account. FLUENT provides an optional implicit body force treatment that can account for this 
effect, making the solution more robust [32].

\subsubsection{Under Relaxation Factor}

Because of the non-linearity of the equation set, the segregated solver uses an under-relation factor to control the computed variables (e.g. pressure) at each iteration. This means that all equations solved using the segregated solver, will have under-relaxation factors associated with them. It is good practice to begin a calculation using the default under-relaxation factors in FLUENT, which are 0.3 and 0.7 respectively for pressure and momentum [32]. If the residuals continue to increase after the first 4 or 5 iterations, the under-relaxation factors should change. The best values for under-relaxation factors obtained are 0.6 and 0.8 for pressure and momentum respectively.

\subsubsection{Material Properties}

There are two phases inside the tank, phase1- Air, and phase2- liquid Fuel. The properties of these phases are shown in Figure 3-9:

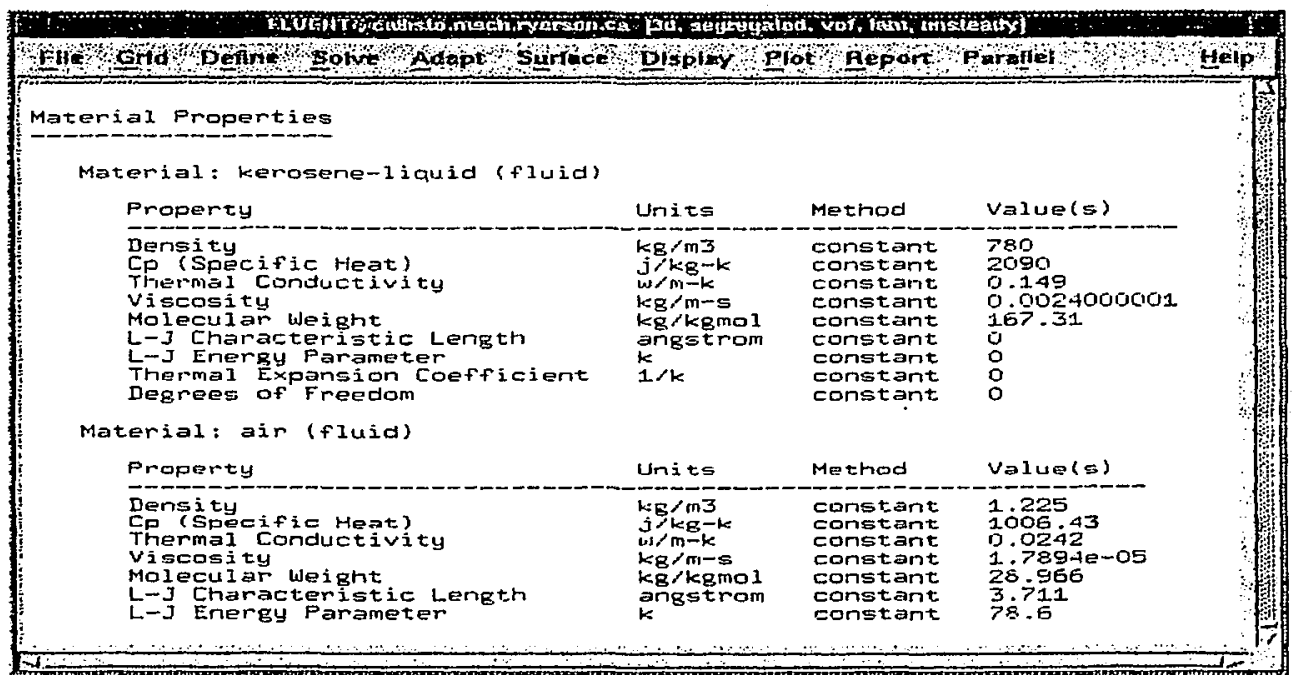

Figure 3-9 Material Properties of two Phases inside the Tank (Air and Fuel). 


\subsubsection{Solution}

During discretization, Body Force Weighted has been selected for pressure, the Pressure Implicit with Splitting of Operators, PISO for Pressure- Velocity Coupling, and First Order Upwind for Momentum [32,34].

$>$ PISO: The pressure Implicit with Splitting of Operators (PISO) pressurevelocity coupling scheme, is based on the higher degree of the approximate relation between the correlations for pressure and velocity. As a result, the calculation must be repeated until the balance is satisfied. To improve the efficiency of this calculation, the PISO algorithm performs two additional correlations: neighbor correlation and skewness correlation.

$>$ Neighbor Correlation: The main idea of the PISO algorithm is to move the repeated calculations required by the solution stage of the pressurecorrelation equation. After one or more additional PISO loops, the correlated velocities satisfy the continuity and momentum equations more closely. This iterative process is called a momentum correlation or neighbor correlation [32].

Skewness Correlation: For meshes with some degree of skewness, the approximate relationship between the correlation of mass flux at the cell face and the difference of the pressure correlations at the adjacent cells is very rough. Since the components of the pressure-correlation gradient along the cell faces are not known in advance, an iterative process similar to the PISO neighbor correction described above is desirable. After the initial 
solution of the pressure-correlation equation, the pressure-correlation gradient is recalculated and used to update the mass flux correlations. This process, which is referred to as skewness correlation, significantly reduces convergence difficulties associated with highly distorted meshes.

When first-order accuracy is desired, quantities at cell faces are determined by assuming that the cell-center values of any field variable represent a cell-average value and hold throughout the entire cell; the face quantities are identical to the cell quantities. Thus the first-order upwinding is selected $[32,34,35]$.

Initialize the Flow Fields: Before starting CFD simulation in FLUENT, an initial for the solution flow field in entire domain must be performed. Initial values relative to the zone Fluid has been set as follows:

Initial pressure value for all fluids $=0$

Initial velocities values for all fluids related to tank velocity are:

$$
V_{x}=0, V_{y}=0, V_{z}=0
$$

Now the discretized model with setting all conditions, initial values, and segregated solution algorithm is ran for interval time steps $\Delta t=0.0025 \mathrm{sec}$, and for $1.1 \mathrm{sec}$ as per acceleration, $a_{x}$, profile:

$$
a_{x}=\left\{\begin{array}{l}
0 \ldots \ldots \ldots \ldots \ldots \ldots \text { for }, t=0 \mathrm{sec} \\
-10 \mathrm{~m} / \mathrm{s}^{2} \ldots \ldots \ldots \text { for }, 0<t \leq 1.1 \mathrm{sec} \\
0 \ldots \ldots \ldots \ldots \ldots . \text { for }, t \geq 1.1 \mathrm{sec}
\end{array}\right.
$$

It is assumed that the vehicle is at rest at time equal zero and after a very short time it starts to move along $x$ direction with linear acceleration equal to $-10 \mathrm{~m} / \mathrm{s}^{2}$ for 1.10 seconds 
(almost equal to a velocity of $40 \mathrm{~km} / \mathrm{h}$ ) then the speed becomes constant. Simulation is ran for different fuel filled depths in the tank, \%25- shallow, \%50-intermediate, and $\% 75$-deep respectively.

\subsection{Sloshing Simulation Result}

Upon completing the simulation, the location of free surface fluid (fuel), and hydrodynamic pressures are determined, and consequentily the impact loads with respect to the area surface of the tank's wall are obtained.

These impact loads on the fuel tank walls from analysis of liquid sloshing simulation inside the moving tank have been reported.

The snapshot for absolute pressure profile of FE simulation of the shallow, intermediate, and deep fuel sloshing (25\%-50\%-75\%) during accelerating in time 0.05 and $1 \mathrm{sec}$ in $\mathrm{x}$-direction of rectangular tank is presented in Fig 3-10.

The liquid locations snapshot of FE simulation of the shallow, intermediate, and deep fuel sloshing during accelerating in time 0.05 and $1 \mathrm{sec}$ is presented in Figure 3-11. As fan be seen from Figure 3-11, the maximum pressure applied on the side wall of the $\operatorname{tank}$ for $25 \%-50 \%$ and $75 \%$ fuel filled are $3000 \mathrm{~Pa}, 4000 \mathrm{~Pa}$, and $5000 \mathrm{~Pa}$ respectively. The operating pressure is assumed to be 1 atm or $100 \mathrm{kpa}$. 


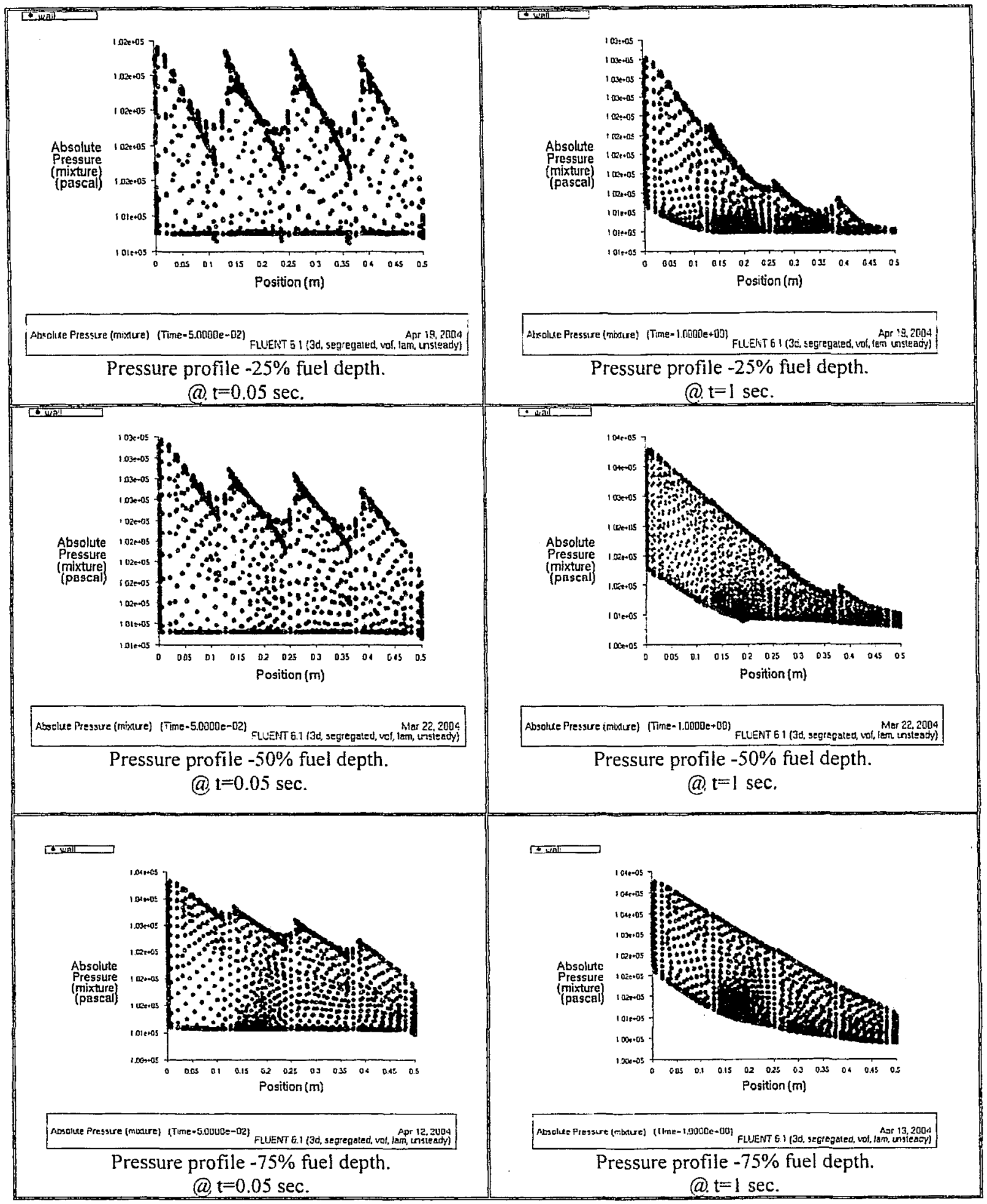

Figure 3-10 The Pressure Profile snapshots of FE Results of the Shallow, Intermediate, and Deep Fuel Sloshing in a Baffled Tank. 


\begin{tabular}{|c|c|}
\hline 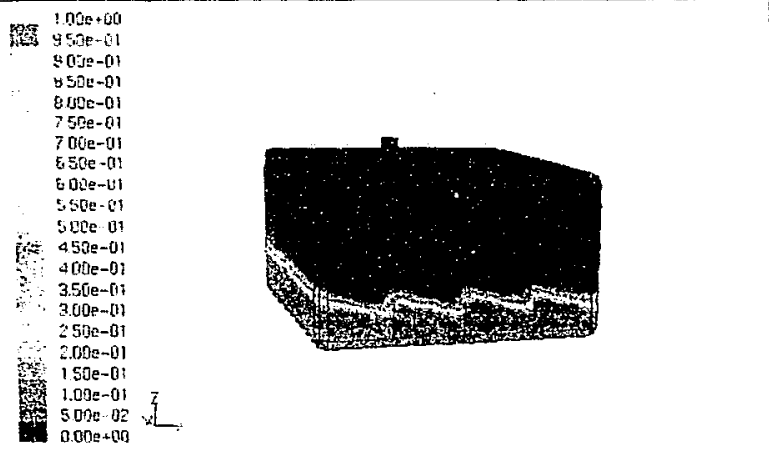 & 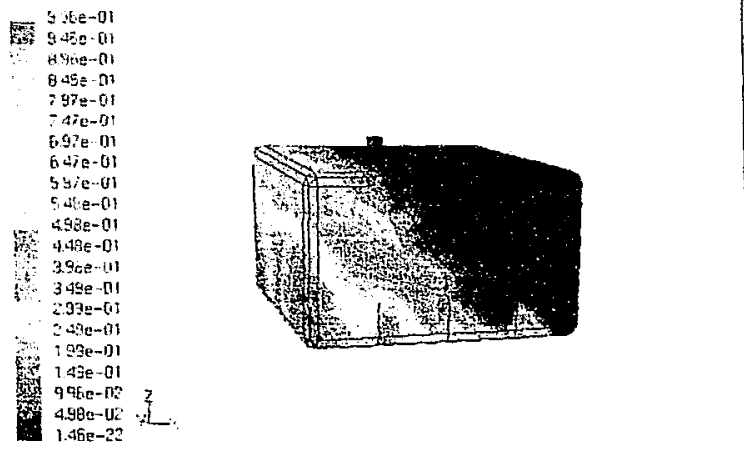 \\
\hline 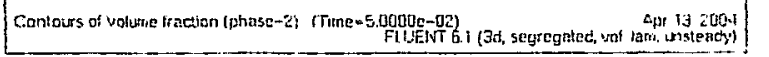 & 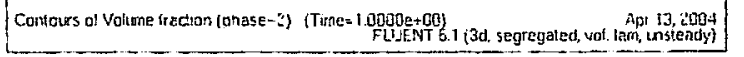 \\
\hline $\begin{array}{l}\text { Fuel location }-25 \% \text { fuel depth. } \\
\qquad \mathrm{t}=0.05 \mathrm{sec} \text {. }\end{array}$ & $\begin{array}{l}\text { Fuel location }-25 \% \text { fuel depth. } \\
\qquad \mathrm{t}=1 \mathrm{sec} \text {. }\end{array}$ \\
\hline 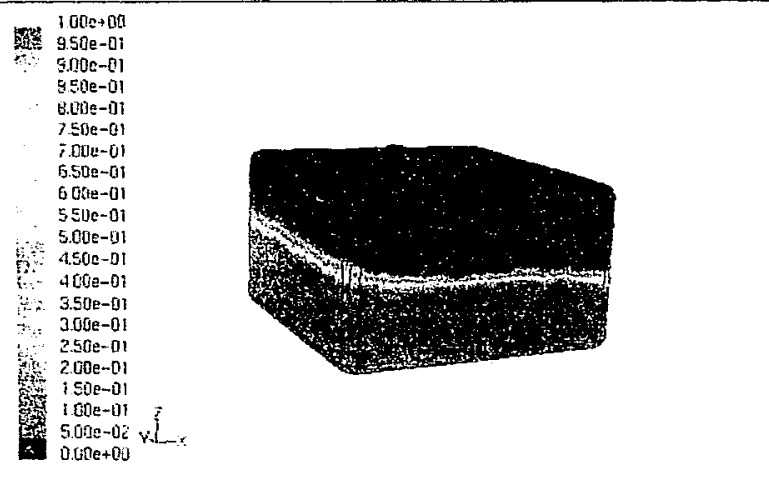 & 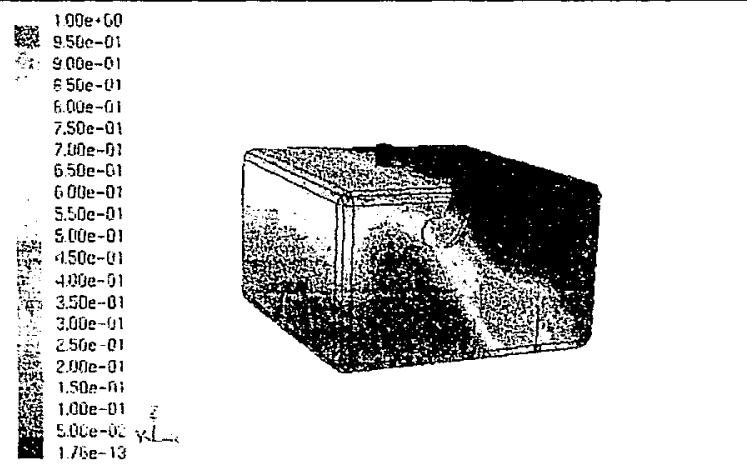 \\
\hline 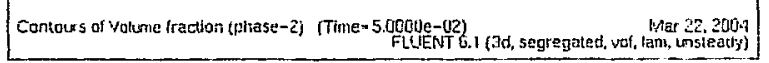 & 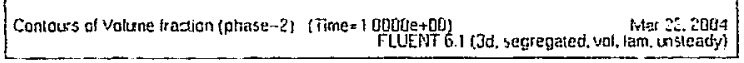 \\
\hline $\begin{array}{l}\text { Fuel location }-50 \% \text { fuel depth. } \\
\qquad \text { (a) }=0.05 \mathrm{sec} \text {. }\end{array}$ & $\begin{array}{l}\text { Fuel location }-50 \% \text { fuel depth. } \\
\qquad, \mathrm{t}=1 \mathrm{sec}\end{array}$ \\
\hline 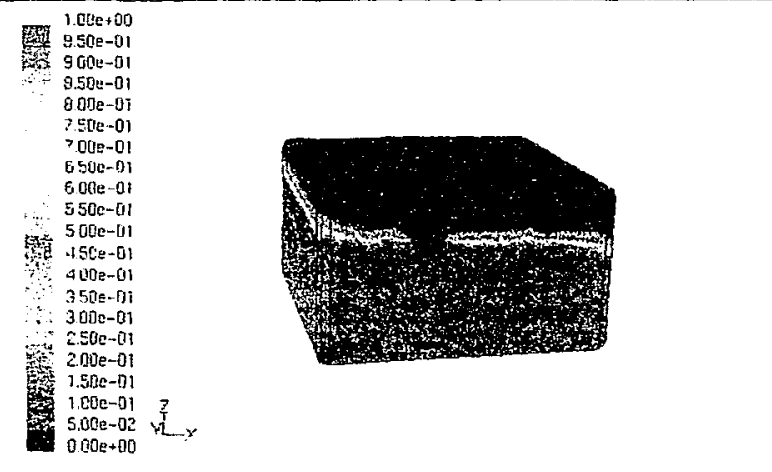 & 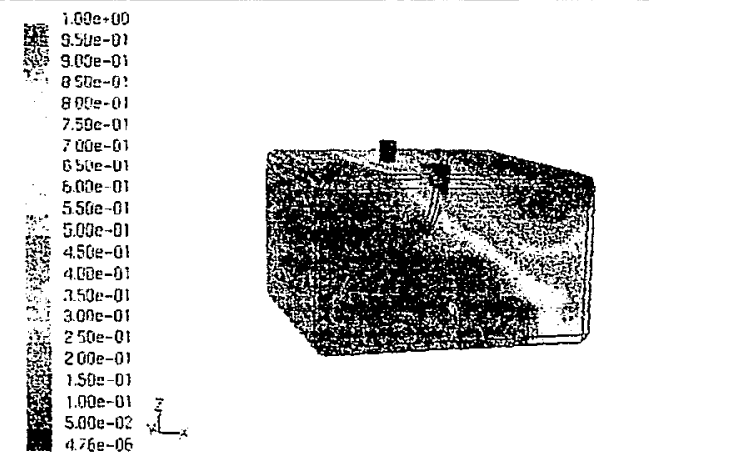 \\
\hline 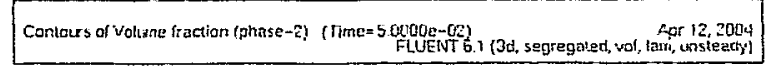 & 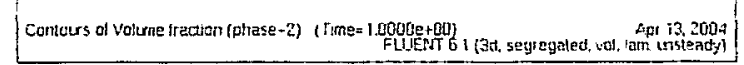 \\
\hline $\begin{array}{l}\text { Fuel location }-75 \% \text { fuel depth. } \\
\qquad(0)=0.05 \mathrm{sec}\end{array}$ & $\begin{array}{l}\text { Fuel location }-75 \% \text { fuel depth. } \\
\text { (a) } t=1 \mathrm{sec}\end{array}$ \\
\hline
\end{tabular}

Figure 3-11 The Fuel Locations Snapshots of FE Result of the Shallow, Intermediate, and Deep Fuel Sloshing in a Baftled Tank. 
Table 3-1 compares the forces exerted on the tank wall. As can be seen the maximum loads are $550(\mathrm{~N}), 322(\mathrm{~N})$ and $169(\mathrm{~N})$ for $75 \%, 50 \%$, and $25 \%$ fuel depth respectively. Figure 3-12 illustrates all three forces curves in one chart with same scale. This fact can be observed from the graphs that sloshing behaviour is similar to vibration behavior in mechanical systems. For fatigue analysis of tank straps, the forces in intermediate fuel filled depth considered because of its average fuel level during driving. Therefore the forces profile for $50 \%$ fuel depth will transfer to fatigue analysis of straps.

\begin{tabular}{|c|c|c|c|}
\hline Time(s) & $\begin{array}{l}25 \% \text {-fuel depth- } \\
\text { Force }(x)-N\end{array}$ & $\begin{array}{l}50 \% \text {-fuel depth- } \\
\text { Force }(x)-N\end{array}$ & $\begin{array}{l}75 \% \text {-fuel depth- } \\
\text { Force }(x)-N\end{array}$ \\
\hline 0 & 0 & 0 & 0 \\
\hline 0.05 & 107 & 87 & 188 \\
\hline 0.1 & 137 & 131 & 342 \\
\hline 0.15 & 149 & 219 & 550 \\
\hline 0.2 & 158 & 315 & 489 \\
\hline 0.25 & 166 & 322 & 500 \\
\hline 0.3 & 169 & 279 & 421 \\
\hline 0.35 & 163 & 248 & 423 \\
\hline 0.4 & 157 & 236 & 425 \\
\hline 0.45 & 153 & 237 & 428 \\
\hline 0.5 & 152 & 248 & 432 \\
\hline 0.55 & 156 & 275 & 438 \\
\hline 0.6 & 159 & 300 & 446 \\
\hline 0.65 & 158 & 301 & 450 \\
\hline 0.7 & 156 & 292 & 448 \\
\hline 0.75 & 154 & 280 & 442 \\
\hline 0.8 & 151 & 270 & 433 \\
\hline 0.85 & 149 & 268 & 422 \\
\hline 0.9 & 148 & 271 & 410 \\
\hline 0.95 & 148 & 275 & 399 \\
\hline 1 & 150 & 278 & 396 \\
\hline 1.05 & 151 & 280 & 399 \\
\hline 1.1 & 153 & 282 & 404 \\
\hline
\end{tabular}

Table 3-1 Slosh Forces on the Wall of the Fuel Tank of an Accelerating Car for 25\%$50 \%$-and $75 \%$ Fuel Depths. 


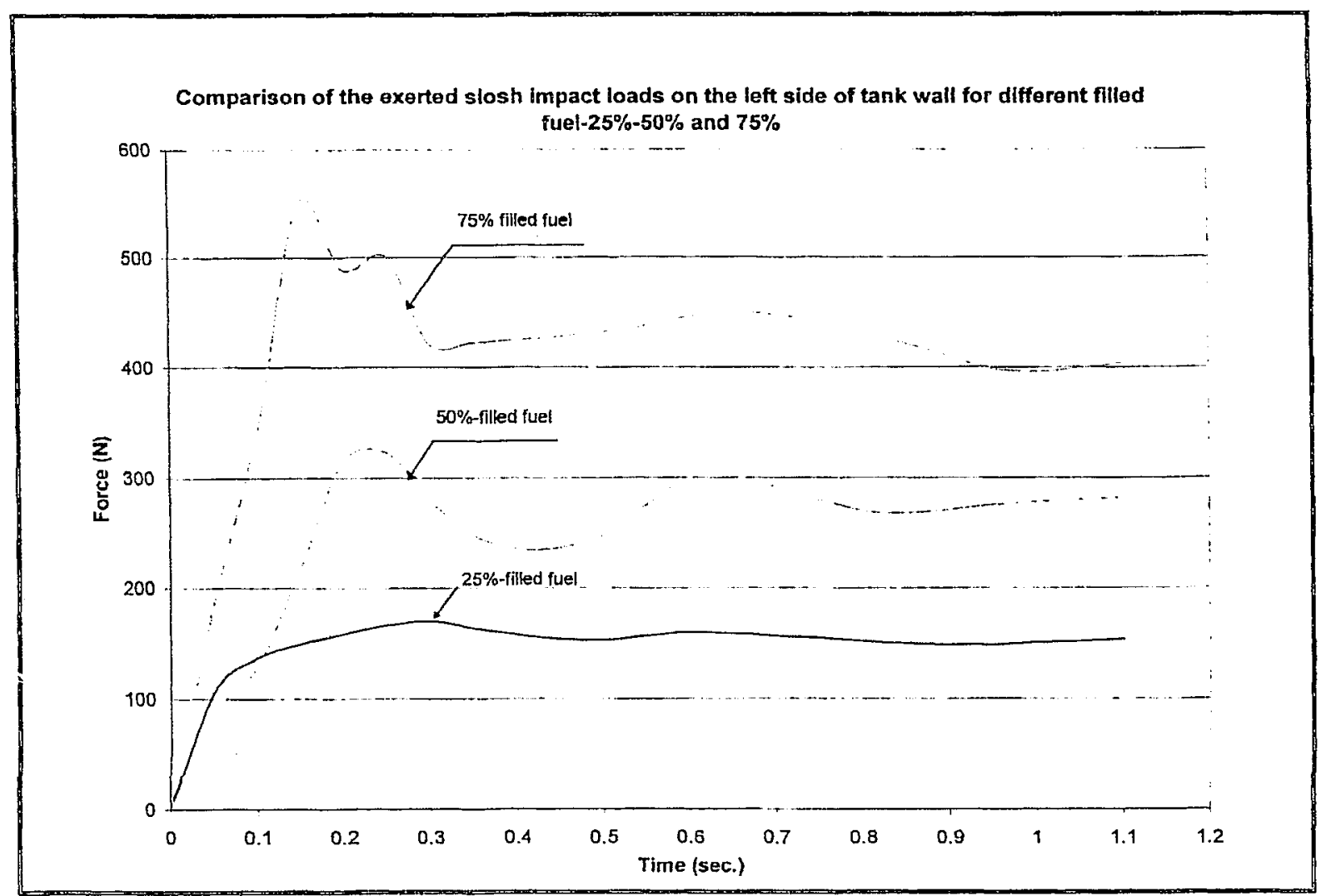

Figure 3-12 Comparison Between the Exerted Slosh Forces on the Tank Wall in the $x-$ direction for $25 \%-50 \%$ and $75 \%$ of the Tank Volume Filled with Fuel.

\subsection{Comparison of Sloshing Results in Baffled and Un-baffled Tanks}

A common method to reduce sloshing loads is to use separating walls known as baffles. Modern design of road tankers widely uses baffles in tanks to reduce the effects of the slosh loads on tank structure and its stability [3]. The limited number of investigations related to road tankers deal mostly with the coupling effect of liquid-wall interactions. Herein, the fuel sloshing in baffled and un-baffled rectangular tanks and influence of baffles on the magnitude of liquid forces are investigated. For both cases, fill levels of $25 \%$ and $50 \%$ with respect to the container height are considered. Both tanks have been excited with same rigid body acceleration profile, $a_{x}$, and same numerical simulations were 
employed to analyze the fluid flow. Figures 3-13 and 3-14 show the simulation results and compare the pressure profiles and the fuel locations for both baffled and un-baffled tanks with $50 \%$ fuel depth at same time.

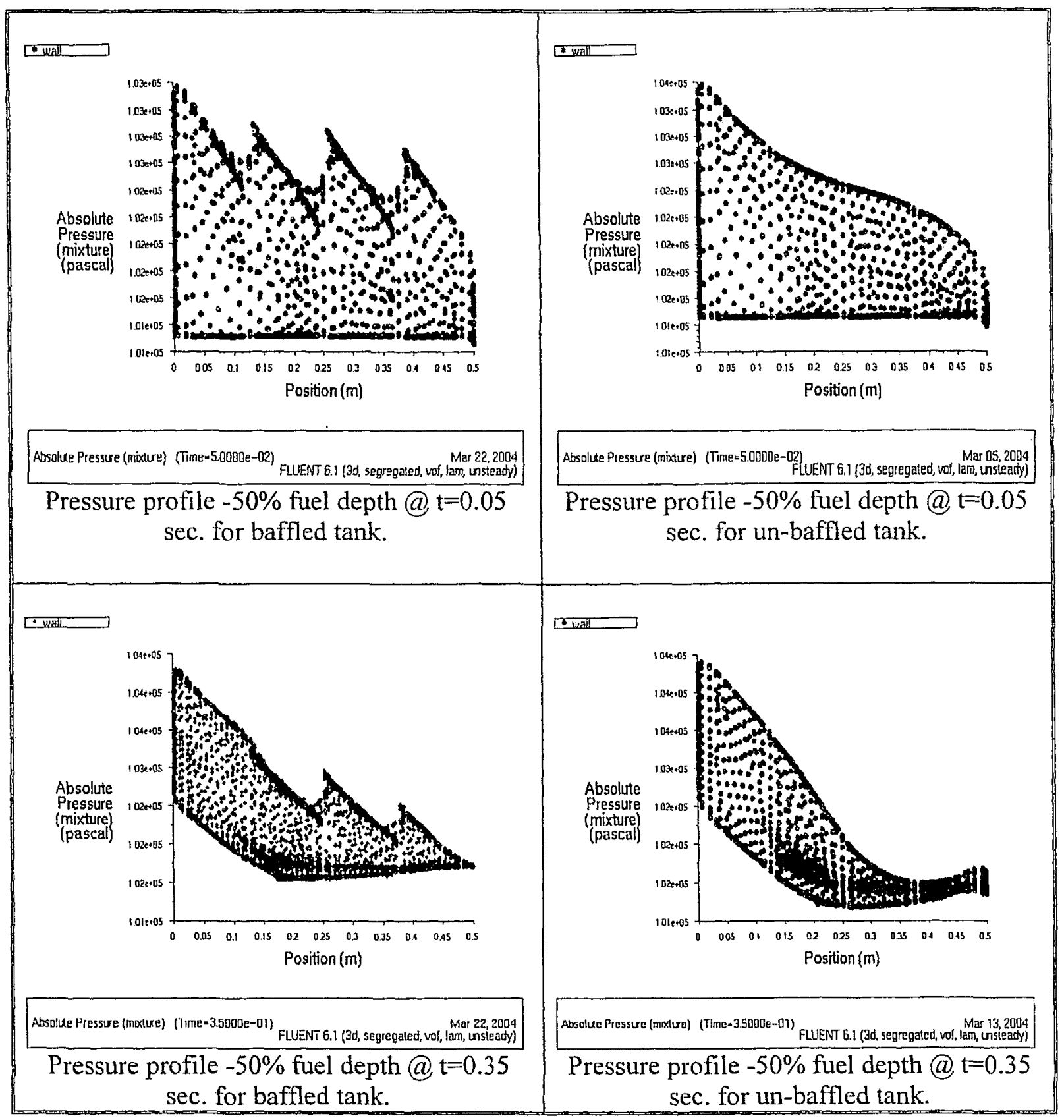

Figures 3-13 Sloshing Pressure Profiles for Baffled and Un-baffled Tanks. 


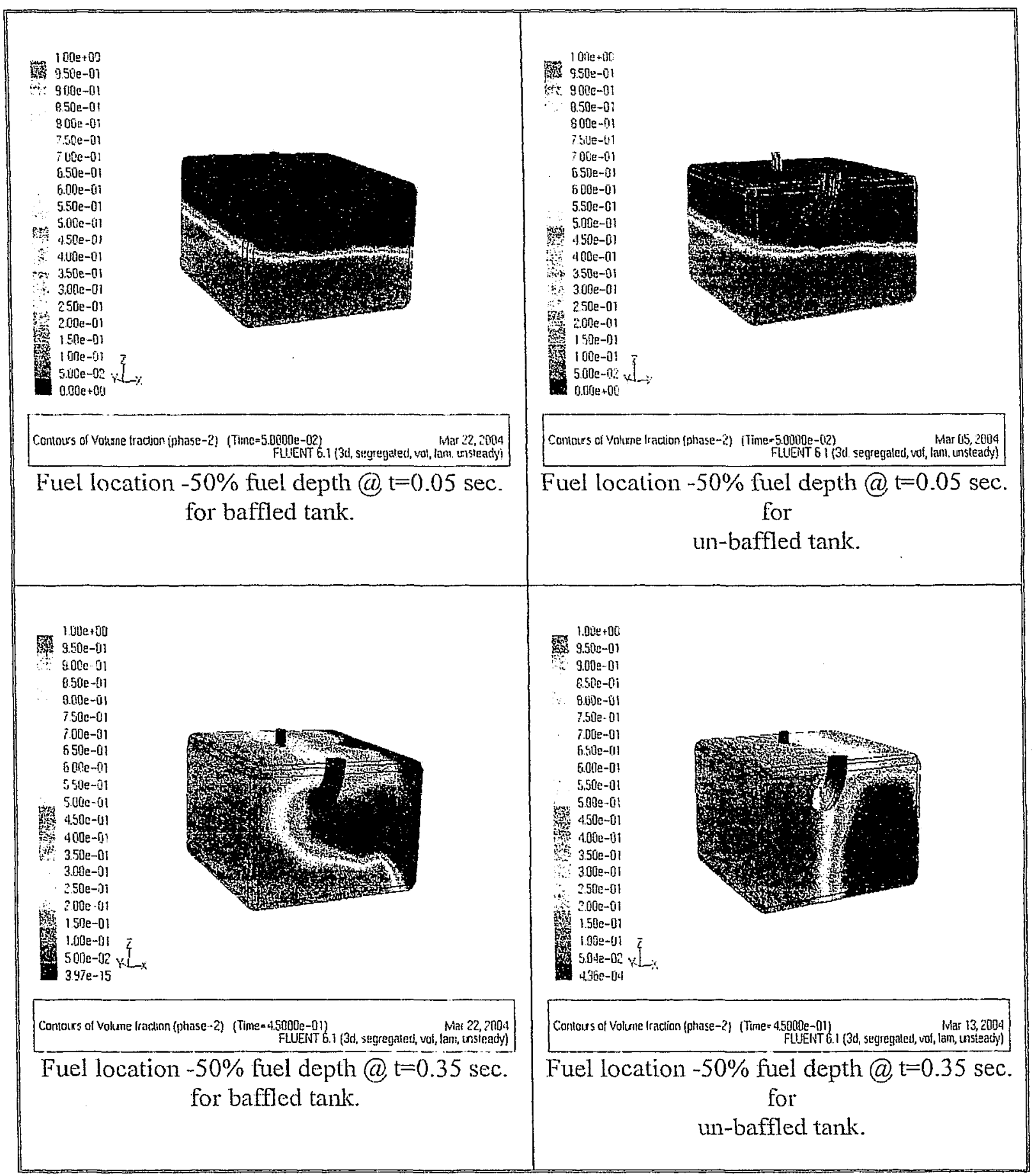

Figures 3-14 Fuel Locations for Baffled and Un-baffled Tanks. 
Figure 3-15 compares the exerted slosh forces on the wall of the baffled and un-baffled tank as same boundary conditions. As can be seen, the forces in un-baffled tank are higher than the forces in baffled tank at the same time. It implies that baffles have damping role in sloshing and three baffles can reduce about $20 \%$ of slosh impact loads. Another fact can be determined from Figure 3-15 is that baffles effect in deep liquid motion is less than those of the shallow fuel depth.

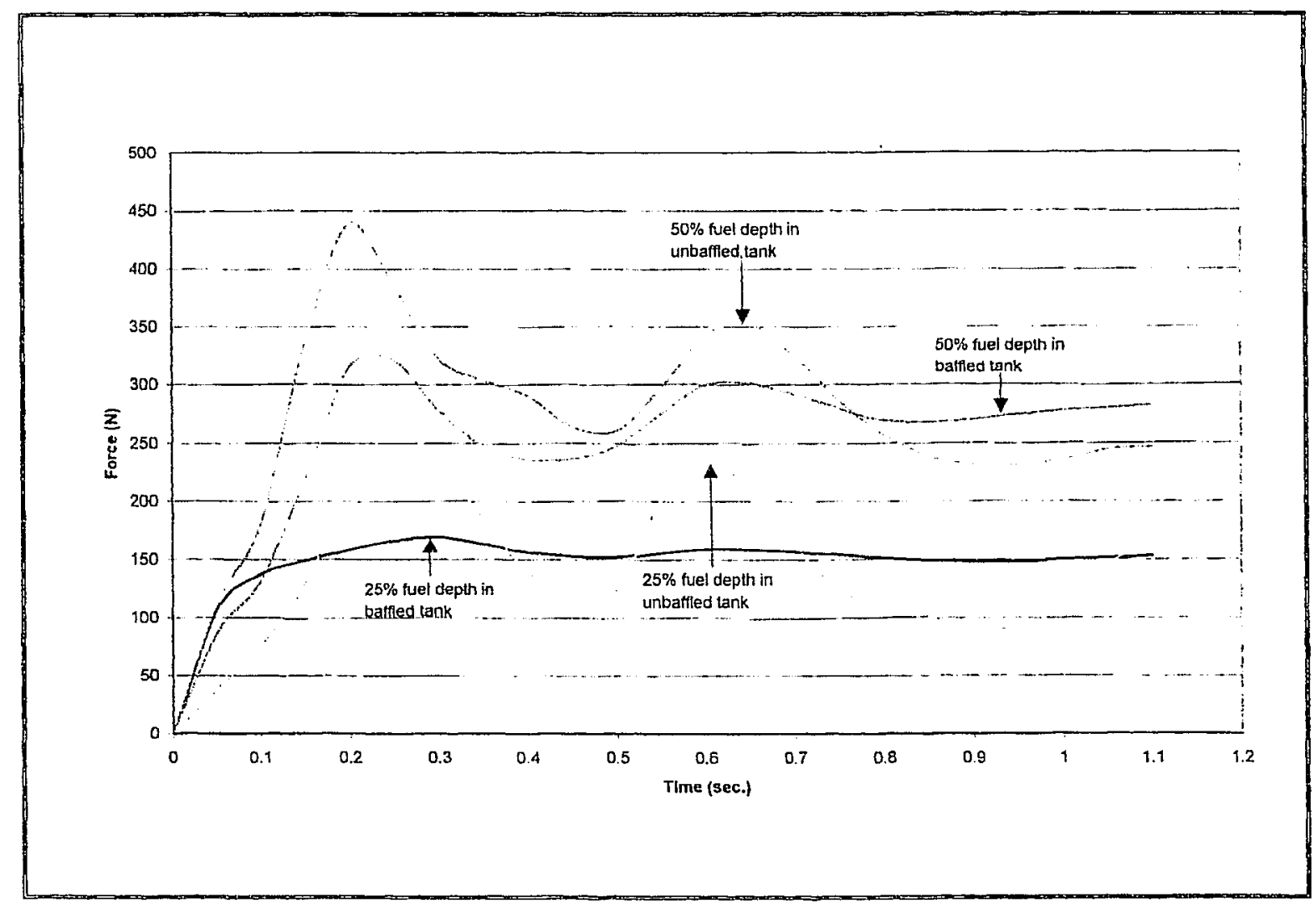

Figure 3-15 Comparison of Slosh Forces in Baffled and un-Baffled Tanks.

Results also show that the oscillations on the curves which represents the sloshing effects is much less compared with the shallow water. 


\section{CHAPTER-4 EQUIVALENT MECHANICAL MODELS FOR SLOSHING SIMULATION}

In this chapter equivalent mechanical models are presented to simulate and verify the result obtained from FE sloshing simulation in moving fuel tank, which has been described numerically and analytically in the previous chapter.

\subsection{Introduction}

Modeling of sloshing based on the finite element method is a powerful tool to analyze the free surface problem in moving tanks because of its flexibility and ability to model all the complications involved with the analysis of such fuel tanks. However, it is computationally expensive and time consuming. In addition, the equations of motion for such masses are usually included more readily in the overall transfer function of the car than are the equations for a continuously deformable medium such as liquid fuel. For these reasons, it is often advantageous in sloshing to replace the liquid, conceptually, by an equivalent mechanical system $[8,9,27,28]$.

The main point that should be noted about the equivalent mechanical models is that the liquid cannot be completely replaced by a single rigid mass attached to the tank. Instead, a system of spring masses or a pendulum must be included because the oscillating masses are necessary to duplicate the oscillating slosh forces arising from the wave action at the free surface [27]. 


\subsection{Sloshing Forces Analysis}

Generally, the liquid hydrodynamic pressure in moving rigid containers is comprised of two distinct parts. One part is directly proportional to the acceleration of the tank. This part is caused by the fluid moving in unison with the tank. The second part is known as convective pressure and experiences sloshing at the free surface and acting impact forces on the tank walls. This part can be modeled by a set of mechanical components such as pendulum [8].

In Figure 4-1, the slosh forces acting on the moving rectangular tank walls, $F_{\mathrm{I}}$, can be estimated by integrating the fluid hydrodynamic pressure, $P$, distribution over the walls of the tank as follows [9]:

$$
F_{I}=d \int_{-h}^{-1} P(l / 2, z, t) \cdot d z-d \int_{-h}^{-2} P(-l / 2, z, t) \cdot d z
$$

where $h$ is the fluid depth, $z_{1}$ and $z_{2}$ represents the surface elevations close to the tank walls at $x=l / 2$ and $x=-l / 2$ respectively.

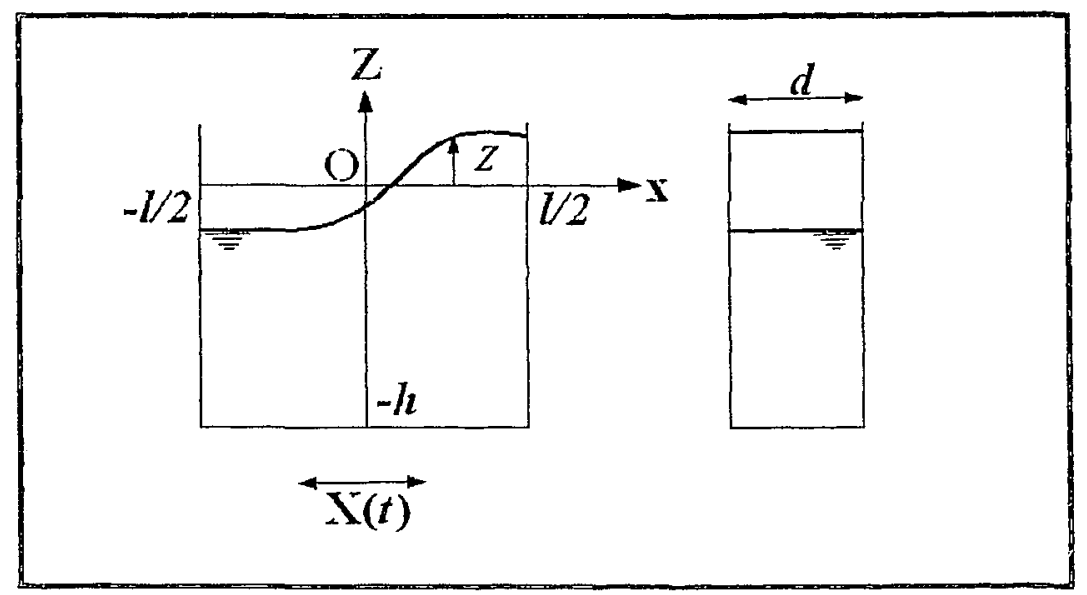

Figure 4-1 Liquid motions in moving Tanks. 
This force, $F_{\mathrm{J}}$, in fuel tank of an accelerating car has been determined numerically in previous chapter. The aim of this section is to simulate this force via an offered equivalent mechanical model approach.

\subsection{Mechanical Analysis of Liquid Sloshing}

Three dynamic regimes are possible during liquid sloshing $[8,9]$, as demonstrated in Figure 4-2:

1-Small oscillations in which the fluid free surface remains planar without rotation, Figure (4-2.a.). This regime can be described by a linear equation for the first asymmetric sloshing mode which is equivalent to a pendulum describing small oscillations such that $\operatorname{Sin} \theta \simeq \theta$.

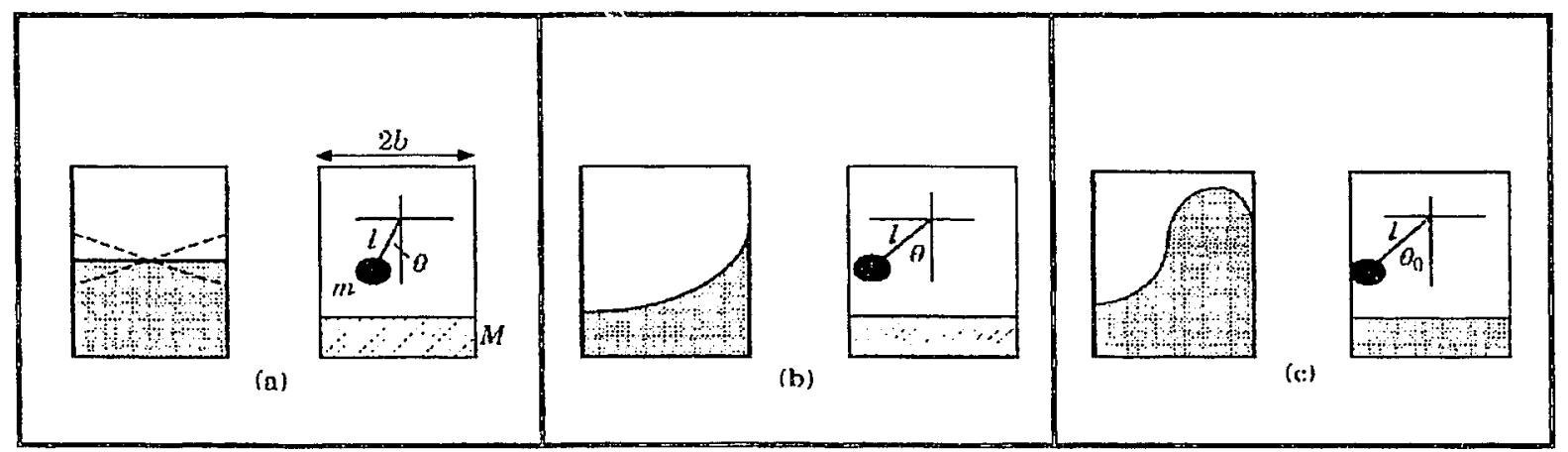

Figure 4-2 Three regimes of Liquid Free Surface motion and their Modeling: (a) Linear Modeling (b) Weakly Non-Linear Modeling (c) Hydrodynamic Impact Pressure.

2- Relatively large amplitude oscillations in which the liquid free surface experiences both non-planar and rotational motions, Figure (4-2.b). This regime is described by a differential equation with weak non-linearity and can be analyzed using the standard perturbation techniques [8]. The equivalent mechanical model is the simple pendulum such 
that $\operatorname{Sin} \theta=\theta-\theta^{3} / 3$ !

3- Strongly non-linear motion, where the non-linearity is mainly due to rapid velocity changes associated with hydrodynamic pressure impacts of the liquid portion close to the free surface, Figure(4.2.c). The velocity changes of the liquid free surface are usually treated as being instantaneous (velocity jumps) and they lead to various strongly non-linear features of the system behavior. This regime can be modeled by a pendulum experiencing impacts with the tank wall [8].

The mathematical modeling of liquid containers supported on elastic structures for strongly non-linear motion has been developed by Ibrahim et al. [8] by considering a liquid container supported by four massless rods of length $L$, which are restrained by four torsional springs $\boldsymbol{k}$ at a base as shown in Figure 4.3. Let $M$ be the total mass of the container including liquid, and $\boldsymbol{m}$ be the equivalent sloshing mass of the first asymmetric mode of the liquid as shown in Figure 4-3. The fluid free surface is modeled as a pendulum of length $l$. The pendulum can reach the walls of the tank if its angle with the vertical axis is $\theta= \pm \theta_{0}$. With reference to Figure $4-3$, the Cartesian co-ordinates of the pendulum and the container positions are:

$$
\begin{aligned}
& x_{p}=L \sin \varphi+l \sin \theta, \\
& z_{p}=L \cos \varphi-l \cos \theta+H \\
& x_{c}=L \sin \varphi, \\
& z_{c}=L \cos \varphi,
\end{aligned}
$$

where the subscripts $p$ and $c$ represent pendulum and container respectively. 


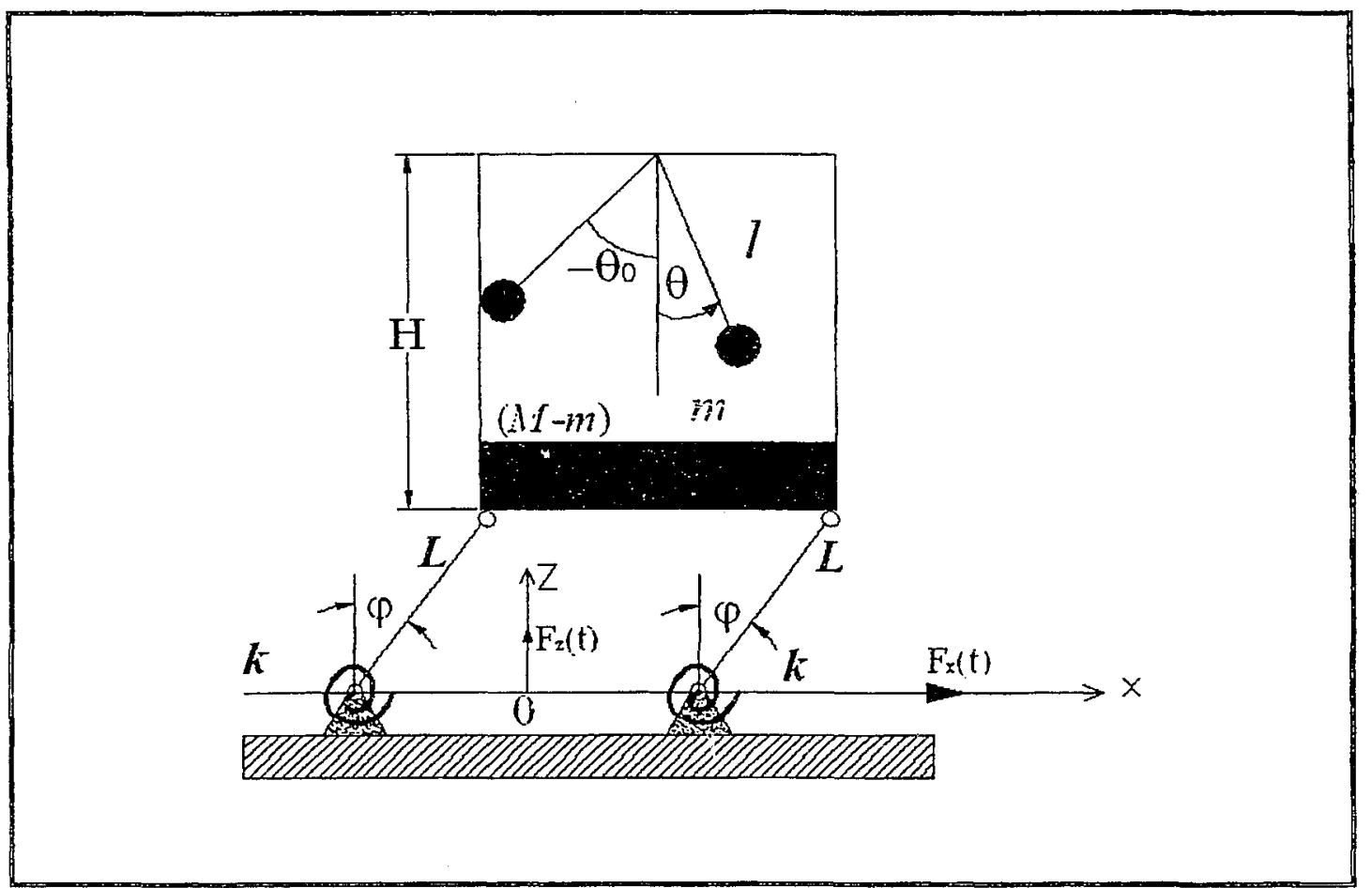

Figure 4-3 Schematic diagram of pendulum model on elastic structure.

The potential energy of the system can be written as:

$$
\begin{gathered}
U=\frac{k \varphi^{2}}{2}+(M-m) g z_{c}+m g z_{p}+U_{l}+c t \\
U=\frac{k \varphi^{2}}{2}+(M-m) g L \cos \varphi+m g(L \cos \varphi-l \cos \theta+H)+U_{l}+c t
\end{gathered}
$$

where $U_{I}=\frac{a \theta_{0}}{2 n}\left(\frac{\theta}{\theta_{0}}\right)^{2 n}(4-6 \mathrm{a})$ : is the potential energy of interaction between the pendulum and the tank wall, which is created by the force of fluid $F_{l}=\frac{d U_{l}(\theta)}{d \theta}(4-6 \mathrm{~b})$. 
The kinetic energy of the system can be written as:

$$
\begin{gathered}
T=\frac{m}{2}\left(\dot{x}_{p}^{2}+\dot{z}_{p}^{2}\right)+\frac{M-m}{2}\left(\dot{x}_{c}{ }^{2}+\dot{z}_{c}{ }^{2}\right) \\
=\frac{M-m}{2} L^{2} \dot{\varphi}^{2}+\frac{m}{2}\left[L^{2} \dot{\varphi}^{2}+2 / L \cos (\varphi+\theta) \dot{\varphi} \dot{\theta}+l^{2} \dot{\theta}^{2}\right]
\end{gathered}
$$

With respect to the Lagrangian equations:

$$
\begin{aligned}
& \frac{\partial L}{\partial \theta}-\frac{d}{d t}\left(\frac{\partial L}{\partial \dot{\theta}}\right)=0 \\
& \frac{\partial L}{\partial \varphi}-\frac{d}{d t}\left(\frac{\partial L}{\partial \dot{\varphi}}\right)=0
\end{aligned}
$$

where $L=T-U$.

and the angle co-ordinates $\theta$ and $\varphi$, the following equations of motion are obtained:

$$
\begin{array}{r}
l^{2} m \ddot{\theta}+l L m \cos (\theta+\varphi) \ddot{\varphi}-l L m \sin (\theta+\varphi) \dot{\varphi}^{2}+l m g \sin \theta+F_{l}=0 \\
l L m \cos (\theta+\varphi) \ddot{\theta}+L^{2} M \ddot{\varphi}-l L m \sin (\theta+\varphi) \dot{\theta}^{2}+k \varphi-L M g \sin \varphi=0
\end{array}
$$

These equations include non-linear terms and non-linear impact load, $F_{I}$.

The angles and time in a scale of the free pendulum oscillations with amplitude $\theta_{0}$ can be measured by introducing the following variables:

$$
\begin{aligned}
& x_{1}=\theta / \theta_{0}, x_{2}=\varphi / \theta_{0}, \quad \mu=m / M, \quad \lambda=l / L, \quad \beta=\frac{b}{L m g \theta_{0}}, \\
& v=\frac{\omega_{L}}{\omega_{l}}=\sqrt{\lambda\left(\frac{k}{M L g}-1\right)}, \quad \omega_{L}=\frac{k-L M g}{M L^{2}}, \quad \omega_{l}{ }^{2}=g / l
\end{aligned}
$$

The parameter $v$ denotes the ratio of two local frequencies; i.e., $\omega_{L}$ is frequency of the 
tank in the absence of the pendulum motion, and $\omega_{l}$ is the frequency of the pendulum if the tank is stationary. The frequency $\omega_{1}$ is equivalent to the fluid first sloshing mode and depends on the tank geometry and fluid depth. With respect to the notations listed in equation (4-12), the equations of motion can be written as:

$$
B \frac{d^{2} X}{d t^{2}}+2 D(x) \frac{d x}{d t}+K x+\beta N(x)=0
$$

where $x=\left[\begin{array}{ll}x_{1} & x_{2}\end{array}\right]^{T}$ and

$$
\begin{gathered}
B=\left(\begin{array}{cc}
\lambda & 1 \\
1 & 1 /(\lambda \mu)
\end{array}\right), \quad D(x)=\left(\begin{array}{cc}
\delta_{1} x_{1}^{2 p} & 0 \\
0 & \delta_{2}
\end{array}\right), K=\left(\begin{array}{cc}
\lambda & 0 \\
0 & v^{2} /(\lambda \mu)
\end{array}\right), \\
N(x)=\left(\begin{array}{c}
x_{1}^{2 n-1} \\
0
\end{array}\right)
\end{gathered}
$$

The natural frequencies of the model can be determined by ignoring of the non-linear terms of equation (4-13) and considering the linear un-damped system:

$$
B \frac{d^{2} x}{d t^{2}}+K x=0
$$

To determine the normal mode the following solution are assumed:

$$
x=H \cos \omega t, \quad H=\left(\begin{array}{l}
h_{1} \\
h_{2}
\end{array}\right)
$$

Substituting equation (4-16) into (4-13) gives a homogeneous set of algebraic equations for the amplitude,

$$
\left(K-\omega^{2} B\right) H=0
$$


and the frequency equation is

$$
\operatorname{det}\left(K-\omega^{2} B\right)=0 \Rightarrow \omega^{4}-\frac{1+v^{2}}{1-\mu} \omega^{2}+\frac{\nu^{2}}{1-\mu}=0
$$

The roots of equation (4-18) give the linear modal frequencies:

$$
\begin{aligned}
& \omega_{1}^{2}=\frac{1+v^{2}+\sqrt{\left(1+v^{2}\right)^{2}+4(1-\mu) v^{2}}}{2(1-\mu)}, h_{2}=\frac{\lambda \mu \omega_{1}^{2}}{v^{2}-\omega_{1}^{2}} h_{1}, \quad \text { in-phase mode } \\
& \omega_{1}^{2}=\frac{1+v^{2}-\sqrt{\left(1+v^{2}\right)^{2}+4(1-\mu) v^{2}}}{2(1-\mu)}, h_{2}=\frac{\lambda \mu \omega_{2}^{2}}{v^{2}-\omega_{2}^{2}} h_{1}, \quad \text { out-of-phase mode }
\end{aligned}
$$

These modal frequencies are dependent on the local frequency ratio $v=\omega_{L} / \omega_{l}$.

\subsection{Pendulum Model for Liquid Sloshing in Rectangular Tanks}

In Chapter-3, fuel sloshing in a lateral accelerated rectangular tank was analyzed based on FEM and slosh impact loads has been determined. Herein a pendulum model to simulate the fuel sloshing in a rectangular tank is described. Considering the lateral motion of tank, the pendulum model can be simplified as illustrated in Figure 4-4. The equation of motion for this mechanical model is described herein. 


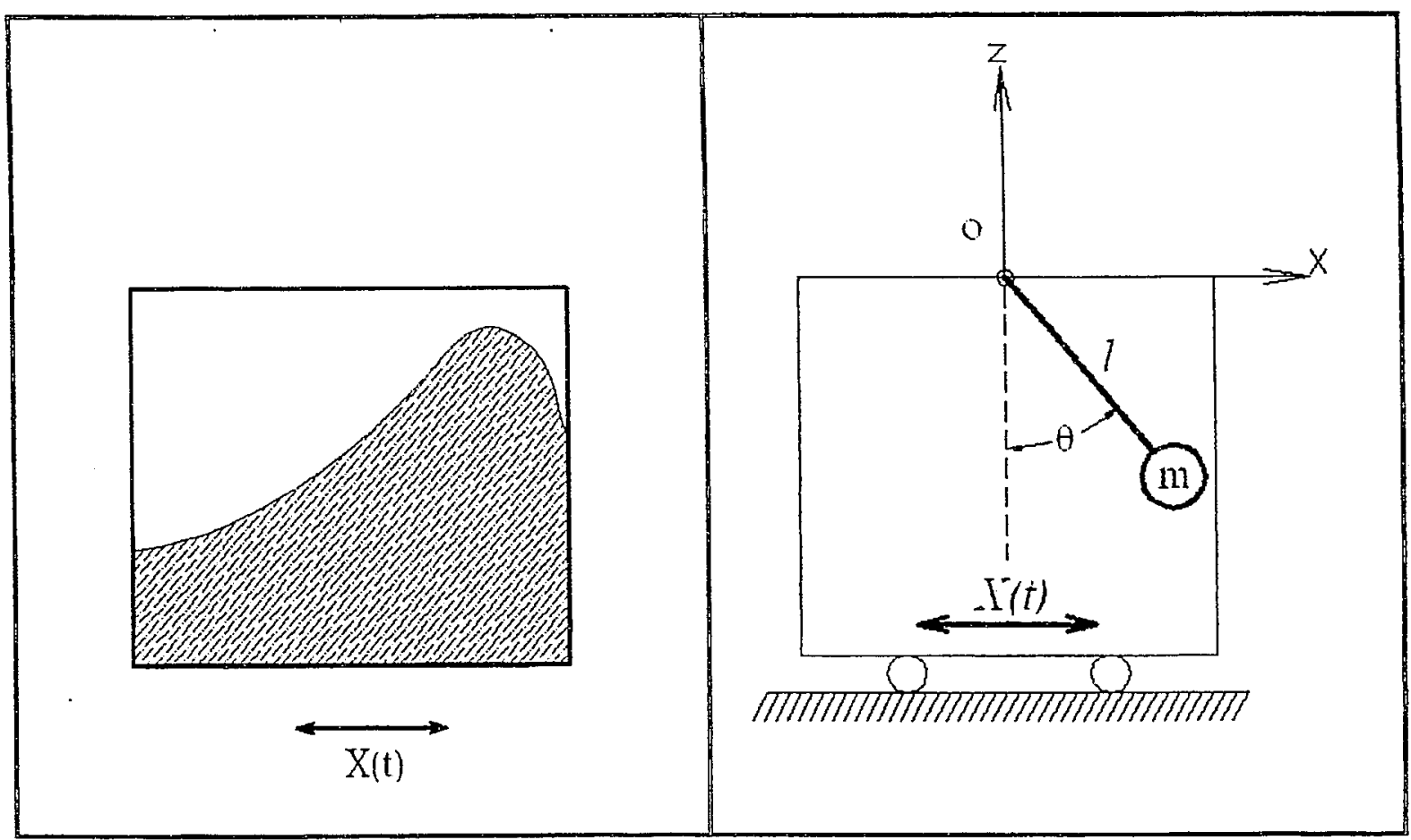

Figure 4.4 A Lateral Excited Tank and a Pendulum Model.

In this model: $m=$ mass of pendulum, $l=$ length of pendulum (mass-less),

$X(t)=$ the tank displacement, $g=$ gravity,

With reference to position of mass:

$$
x=l \sin \theta+X(t), \quad z=-l \cos \theta
$$

and velocity of mass:

$$
\dot{x}=l(\cos \theta) \dot{\theta}+\dot{X}(t), \quad \dot{z}=l(+\sin \theta) \dot{\theta}
$$

With respect to equation (4-22), the equation of motion can be simplified as:

$$
m \ddot{\theta}+(m g / l) \sin \theta+(m \ddot{X} / l) \cos \theta=F_{j}
$$

The effective length of pendulum arm $l$, can be obtained with comparison of first natural frequency of the pendulum $\omega_{l}^{2}=g / l$, and the first asymmetric sloshing mode for 
rectangular tank given by Lamb [37]:

$$
\omega_{R}^{2}=\frac{g \pi}{a} \tanh \left(\pi \frac{h}{b}\right)
$$

where $a=\operatorname{tank}$ width, $b=\operatorname{tank}$ length, and $h=$ liquid depth. Therefore the effective length of pendulum arm is:

$$
l=\frac{a}{\pi} \operatorname{coth}\left(\frac{\pi h}{b}\right)
$$

Equation of motion (4-23) can be re-written as:

$$
\ddot{\theta}+(g \pi / a) \cdot \tanh \left(\frac{\pi h}{b}\right) \cdot \sin \theta+(\pi / a) \cdot \tanh \left(\frac{\pi h}{b}\right) \cdot \ddot{X} \cos \theta=F_{1} / m
$$

In this case, a rectangular tank with $a=0.5 \mathrm{~m}, b=0.5 \mathrm{~m}$ and fuel level is half of the tank's height, $h=0.15 \mathrm{~m}$. It is assumed that the vehicle is at rest at time equal zero and starts to move along the $x$ direction with a linear deceleration equal to $\ddot{X}=-10 \mathrm{~m} / \mathrm{s}^{2}$ for 1.1 seconds (almost equal to a velocity of 40 kilometers per hour in a braking situation) then the speed becomes constant. The fuel inside the tank sloshes and then exerts impact load in the $\mathrm{x}$-direction. Also it is assumed that the pendulum arm is rigid and massless.

The last term in equation (4-26) incorporates the impact load. This pendulum model with equation of motion (4-26) can be treated computationally using the fourth order RungeKutta numerical method in commercial package WORKING MODEL-4D, NASTRAN.

Figure 4-5 illustrates the non-dimensional pendulum mass impact loads and the slosh loads obtained from the FE simulation. This Figure reflects good agreement between the two sets of results. The results show that instead of solving thousands of equations for thousands of elements in fluid domain to find the slosh impact forces, a simple mechanical 
model such as pendulum, can simulate the slosh and determines this load. In addition the functions related to other components involved in the tank such as body and chassis of the car, are easy to apply in the simple mechanical model.

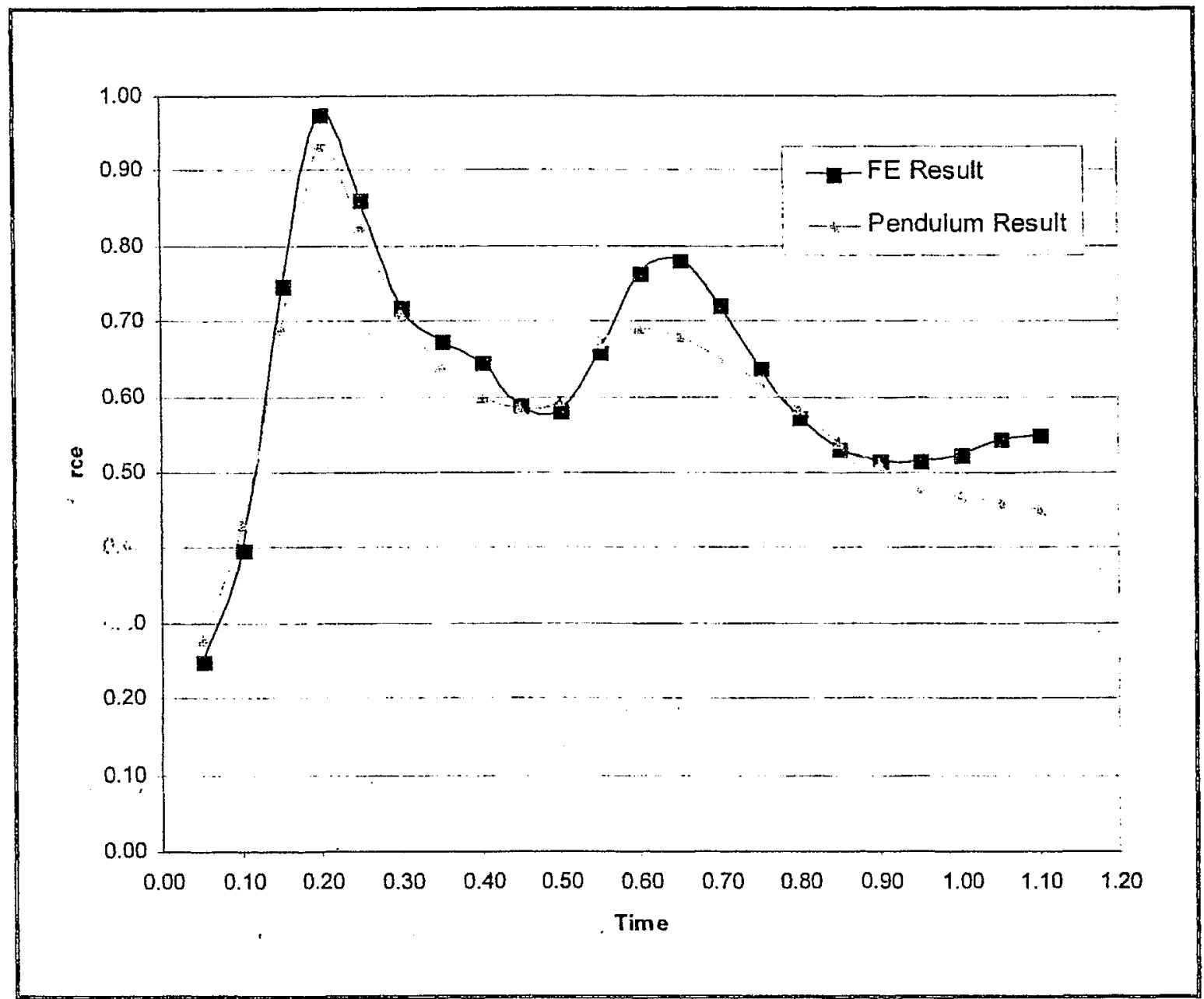

Figure 4-5 Comparison of Impact Forces Exerted on the Wall of the Rectangular Fuel Tank obtained from FE Model and Pendulum Model.

To find the accurate results different simple mechanical models for different sloshing conditions and tank geometries are needed. In the attached appendix to this thesis, another pendulum model for cylindrical tanks (tanker trucks) is presented. 
In this chapter the results obtained from sloshing simulations using finite element method (FEM) have been compared with the results obtained from equivalent pendulum model for rectangular tanks. In both approaches, FE model and pendulum model, the obtained impact forces on the tank walls reflected good agreement at intermediate fuel level. These forces can be used as input data and load-time history for fatigue analysis of fuel tank straps. This is the case of the next chapter work. 


\section{CHAPTER-5 FATIGUE ANALYSIS OF FUEL TANK STRAPS}

\subsection{Overview}

In the automotive industries, due to the general trend of producing lighter vehicle, it has become important to consider the durability of the structure being optimized. This can be achieved by using a minimum fatigue life value as an extra constraint in the optimization process [1]. A critical element is defined as the element with lowest fatigue life value in the FEM of structure that determines the fatigue life of whole structure.

The numerical process of fatigue life analysis of structure in NASTRAN software is started with static load analysis $[5,11,38]$. Static loads (the maximum load in the load-time history) are applied at the same elements of the structure and in the same actual loading direction. The static load analysis produces static stresses and strains. If the maximum static stress obtained from static analysis is less than the yield stress of material of the structure the fatigue life prediction falls in high cycle region and dynamic analysis with respect to load-time history using Stress-Life, S-N, curve has to be considered. If the maximum static stress is higher than yield stress of structure material the fatigue life prediction falls in low cycle region and dynamic analysis with respect to load-time history using Strain-Life, $\varepsilon-N$, curve has to be considered $[5,41]$.

The correct selection of elements is important in FE to obtain reliable results [38]. Elements can be classified as one dimensional (1D), 2D or 3D in FEM. Some of the elements are only applicable to certain situations and some work better than others in a particular situation. Table 5-1 lists the element types and their applications. 


\begin{tabular}{|c|c|c|c|c|}
\hline Types & Topology & $\begin{array}{l}\text { Number of } \\
\text { Nodes }\end{array}$ & $\begin{array}{l}\text { DOF at each } \\
\text { Node }\end{array}$ & Applications \\
\hline 1D Bar & Linear & 2 & $\begin{array}{l}3 \text { ( } 2 \text { translations } \\
+1 \text { rotation) }\end{array}$ & $\begin{array}{l}\text { One- } \\
\text { dimensional } \\
\text { beam problems } \\
\text { where torsional } \\
\text { rigidity is } \\
\text { required. }\end{array}$ \\
\hline \multirow{2}{*}{ 2D Shell } & Tria & 3 & $\begin{array}{l}6 \text { ( } 3 \text { translations } \\
+3 \text { rotations })\end{array}$ & $\begin{array}{l}\text { Three- } \\
\text { dimensional } \\
\text { thin-walled } \\
\text { structures. - } \\
\text { Should be } \\
\text { avoided due to } \\
\text { high stiffness. }\end{array}$ \\
\hline & Quad & 4 & $\begin{array}{l}6 \text { ( } 3 \text { translations } \\
+3 \text { rotations })\end{array}$ & $\begin{array}{c}\text { Three- } \\
\text { dimensional } \\
\text { thin-walled } \\
\text { structures. }\end{array}$ \\
\hline \multirow{2}{*}{ 3D Solid } & Wedge & 6 & $\begin{array}{c}3(3 \\
\text { translations) }\end{array}$ & Solid structures. \\
\hline & Hex & 8 & $\begin{array}{c}3(3 \\
\text { translations) }\end{array}$ & Solid structures. \\
\hline
\end{tabular}

Table 5-1 Typical Element Types and their Applications. 
The discretization of model depends on the geometry of the structure, the loading pattern, and the boundary conditions. First the geometry of structure is modeled and then discretized using appropriate elements.

\subsection{Geometry Modeling}

The Fuel tank is held and fixed to the body of the car with two flat metal strips, which are called straps. On each strap, there are two $1.25 \mathrm{~cm}$ diameter holes that can be bolted to the car body. Thickness of straps is $0.25 \mathrm{~cm}$. The side and bottom wall of fuel tank structure are in contact with straps, therefore all the loads exerted on the tank walls from liquid can be transferred directly to the straps. The geometry model of strap is shown in Figure 5-1.

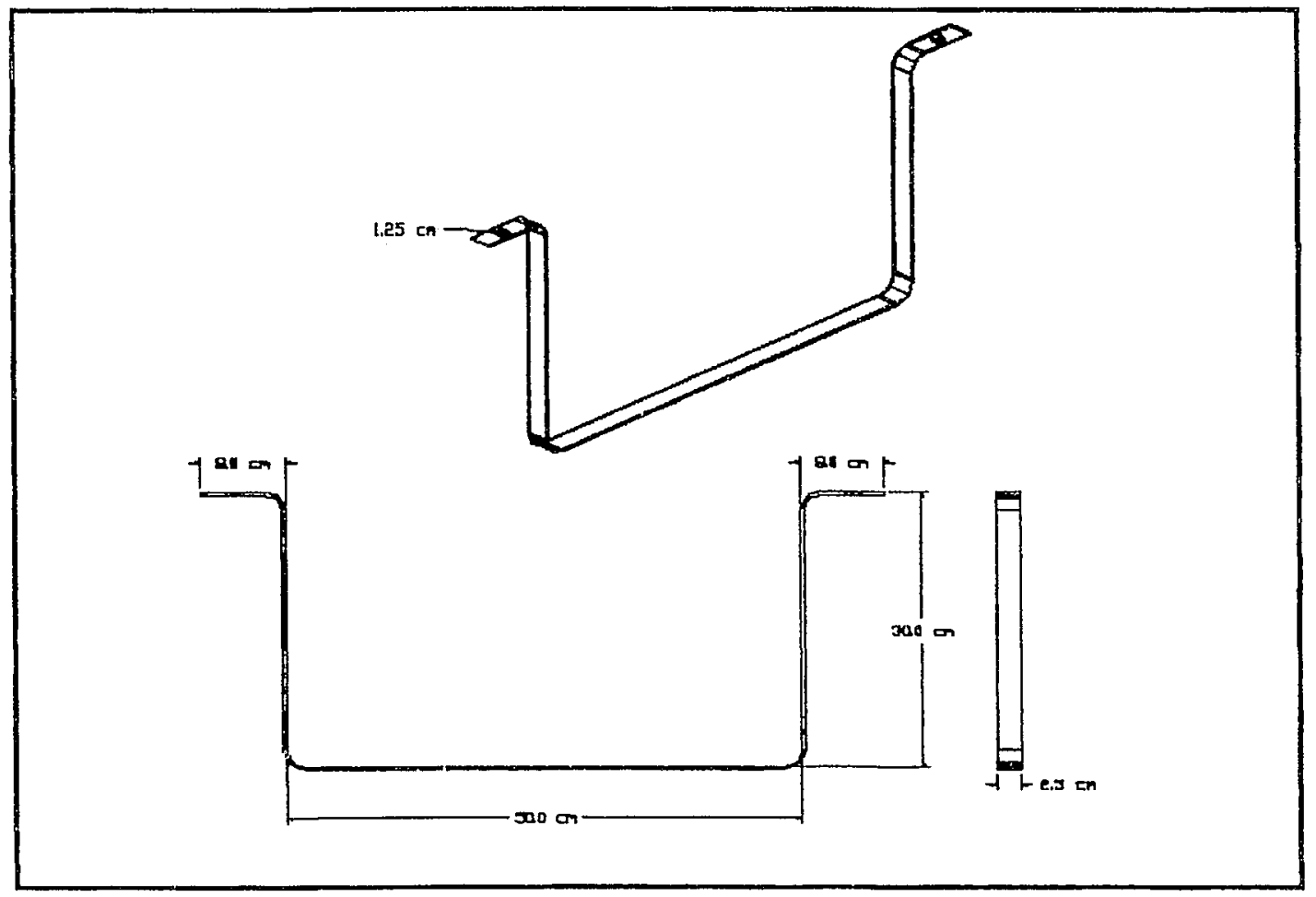

Figure 5-1 Strap Geometry Model. 


\subsection{Finite Element Discretizing}

Shell and solid elements are widely used for discretizing a mechanical components. Since the straps are subjected to both forces and moments in realistic situations, and thickness of straps is too small in relation to the length of straps and remain uniform, the shell element is an ideal choice for discretization. Shell elements also reduce the amount of computation time as compared to solids.

The effect of altering the element edge length from $25 \mathrm{~mm}$ to $5 \mathrm{~mm}$ related to the strap's size, causes the maximum bending stress to approach the exact solution. Indeed, from finite element theory, discretizing the structure into smaller elements should cause the solution to converge to the exact value. However, further refinement of the mesh from $5 \mathrm{~mm}$ to $1 \mathrm{~mm}$ causes a drastic change in the stress. Instead of converging to a value close to the exact solution, the maximum bending stress increases from its original value. The thickness of element is another important parameter that should be considered. In general, a length to thickness ratio of $1: 2$ is appropriate $[11,38]$.

After the surfaces are created, the strap is discretized. The model is meshed using a combination of Quad4-node and Tria3-node shell elements. It is observed that the combination of proper element type and mesh selection would yield static results that are close to realistic solutions. In general, Triangular elements (Tria3) should be avoided due to excessive stiffness. Rectangular elements (Quad4) are ideal for shell mesh. However, in components consisting of holes, it is very difficult to avoid Tria3 elements. It should be noted that a model in which the total number of Tria3 is less than five percent of the total 
number of elements in the model yields conservative results [41]. Figure 5-2 shows the discretized shell model with Tria3 and Quad4 elements with an element edge length of $5 \mathrm{~mm}$ and element thickness $2.5 \mathrm{~mm}$. In this case, the length to thickness ratio is $1: 2$ and this is therefore acceptable. After discretization of the model is completed, several geometry checks are performed in PATRAN to verify whether the elements qualities are acceptable.

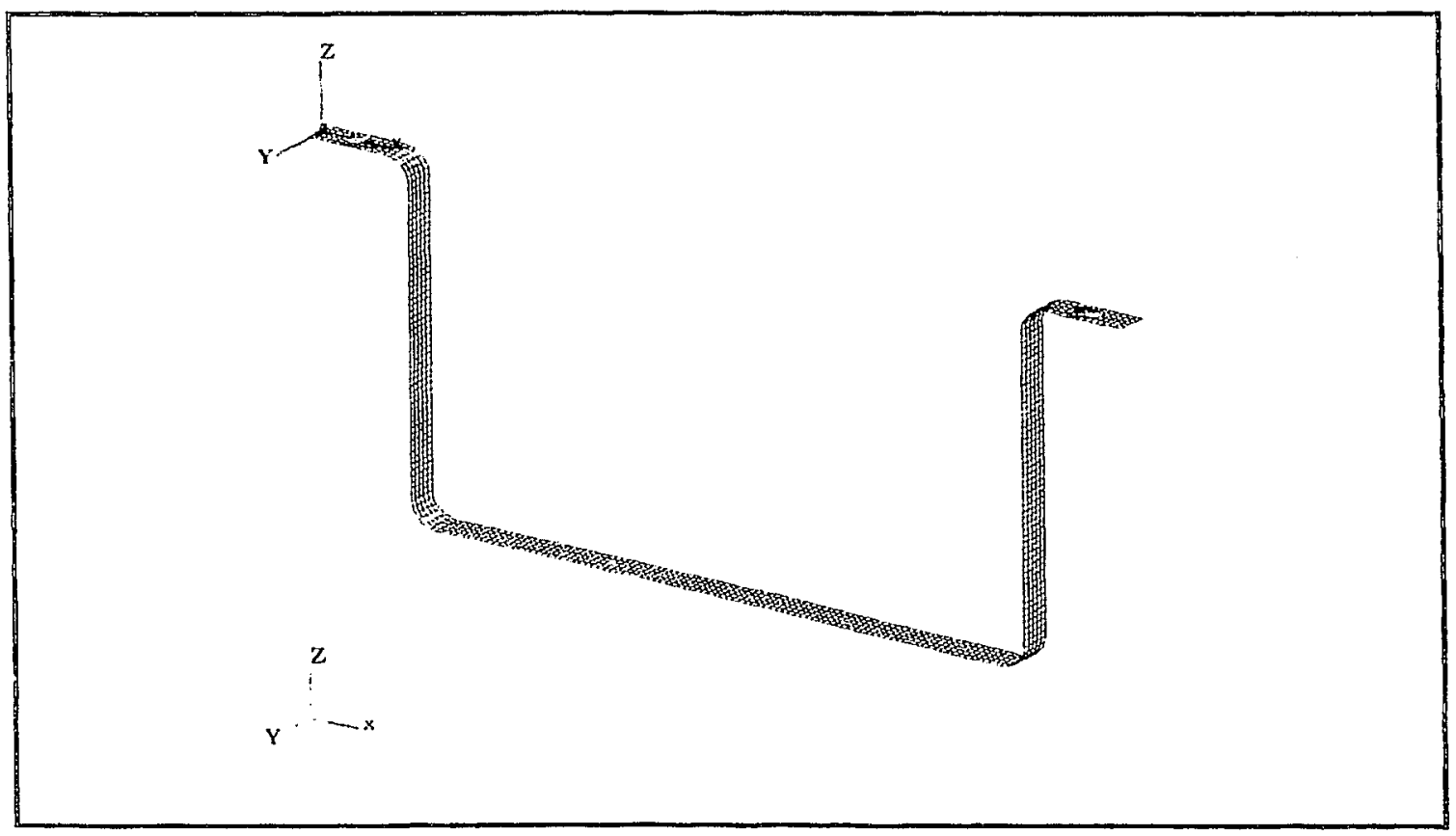

Figure 5.2 Discretized Model of the Strap.

\subsection{Boundary Conditions}

The strap is subjected to a combination of slosh and weight loads. Therefore, the model is built for two different loading conditions, sloshing loads in the x-direction and weight loads in the z-direction. The maximum slosh loads for intermediate slosh depth obtained from simulation is $322(\mathrm{~N})$ and weight load includes of the tank weight plus liquid 
weight. Considering the size of the tank, density of the material of the tank and liquid fuel $7,800 \mathrm{~kg} / \mathrm{m}^{3}$ and $780 \mathrm{~kg} / \mathrm{m}^{3}$ respectively, the total weight load is obtained as follows:

Tank weight: $[4 \times(0.5 \times 0.3)+2 \times(0.5 \times 0.5)] 0.0015 \times 7800 \times 9.81=129$

where thickness of the tank shell $=0.0015 \mathrm{~m}$ and acceleration due to gravity $\mathrm{g}=9.81 \mathrm{~m} / \mathrm{s}^{2}$.

Liquid weight: $(0.5 \times 0.5 \times 0.15) \times 780 \times 9.81=287$

Total weight: $129+287=416$

It is assumed that the weight load in the z-direction is always constant, therefore there is no load-time history for load in the z-direction. Considering the tank is supported with two straps, the loads in each direction should be applied in half. Consequently, the pressure load applied on the each strap in the $\mathrm{x}$-direction for static analysis is:

$(322 \div 2) \div(0.3 \times 0.025)=21.47 \quad \mathrm{kpa}$

and in the z-direction:

$$
(416 \div 2) \div(0.5 \times 0.025)=16.64 \quad \mathrm{kpa}
$$

The strap was fixed in all degree of freedom (DOF) for displacement and rotations around the holes. Figures 5-3 and 5-4 show the strap with loads transferred from the tank and fixed nodes around the holes. 


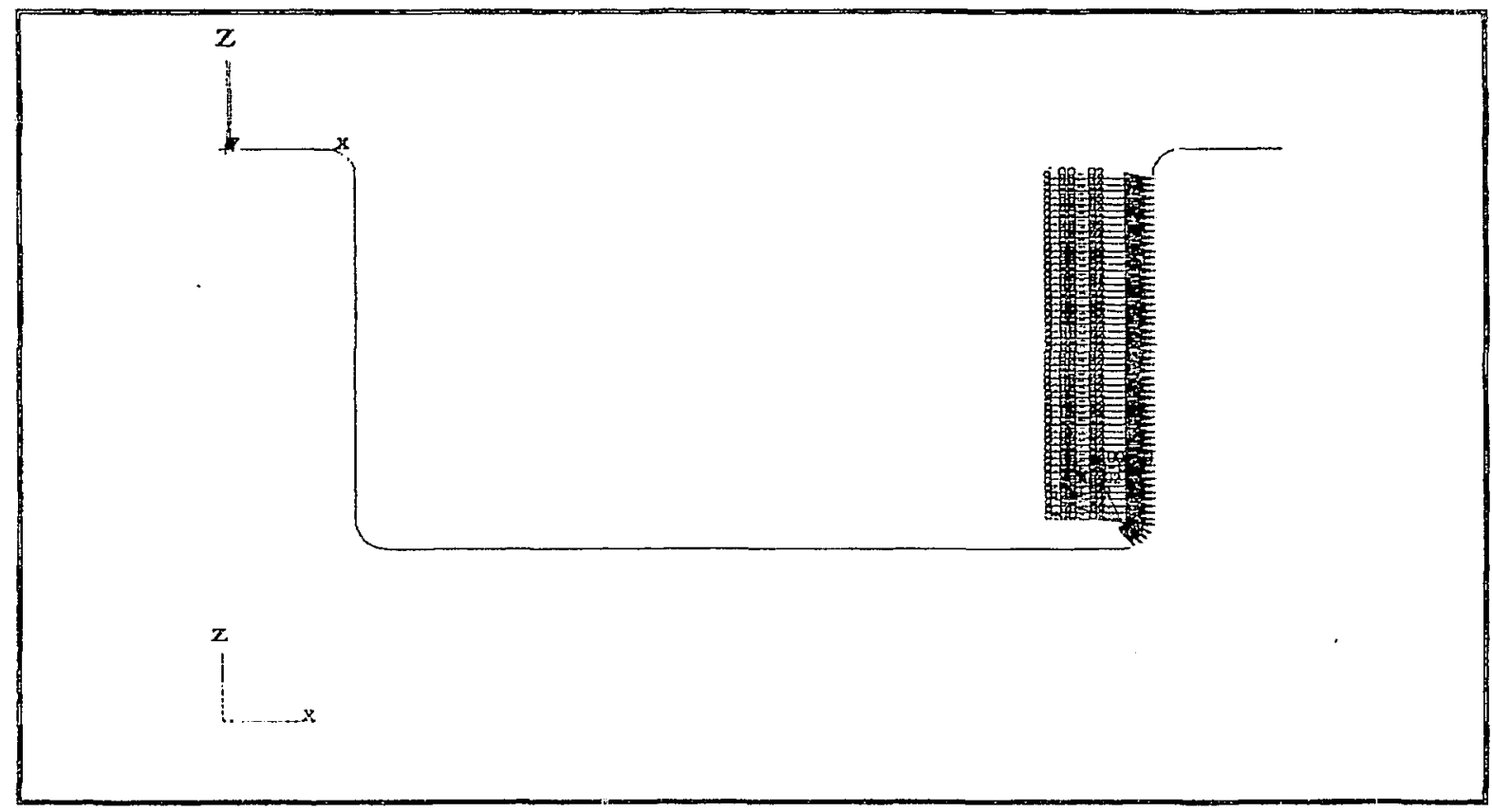

Figure 5-3 Maximum Slosh Loads applied on the Strap for Static Analysis.

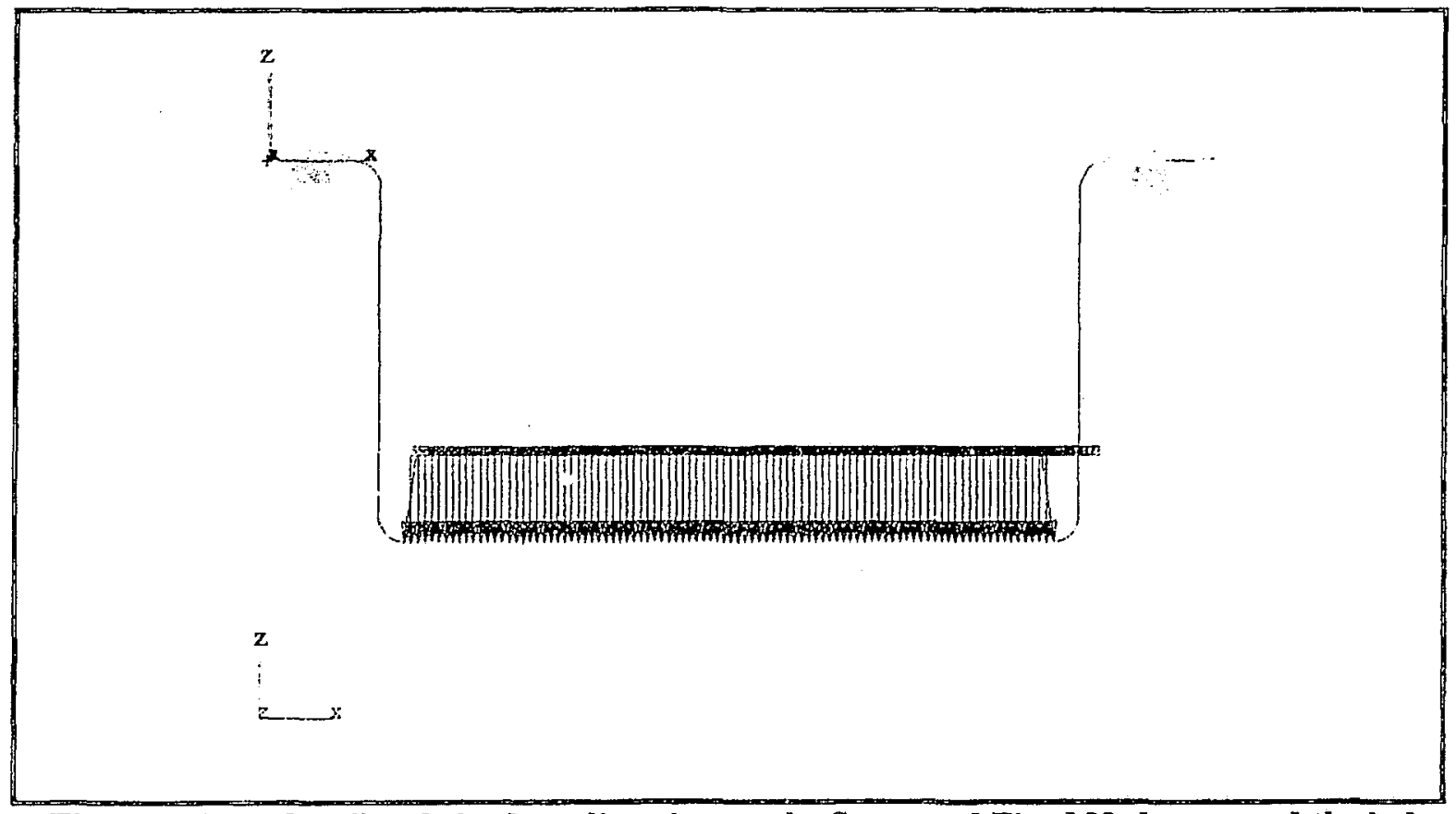

Figure 5-4 Applied Loads in the z-direction on the Strap and Fixed Nodes around the boles. 


\subsection{Finite Element Static Load Analysis: Analysis Parameters}

A.s previously mentioned, the objective of this research is to predict fatigue life in the strap subjected to slosh impact loads. It is therefore necessary to calculate the stresses and strains in a static analysis and use these as input to calculate fatigue life. Before implementing a static analysis, geometric and material properties need to be assigned to all the elements in the strap. The geometric properties in this case are self-defined when shell elements with a constant edge are used. On the other hand, material properties have to be defined in all cases. In this case, the material used is low carbon steel SAE 1006 or mild steel. The desired static results from FEA are used as input data to calculate fatigue life. Therefore it is important :o use the elasto-plastic properties of the material, since it is unknown at this point whether the stresses and strains will fall in the high or low cycle region.

The pressure loads calculated in the previous section are applied on the strap to simulate static analysis and calculate the static stresses using MSC.NASTRAN.

\subsection{Static Simulation Results}

In this section, the stress contour plots obtained from static analysis are displayed. FEA generates maximum and minimum principal stresses (i.e., tensile and compressive stresses). It is important at this point to analyze each stress effectively and use the most critical one as input to calculate life. Usirg improper stress values will result in inaccurate fatigue life prediction.

Figures 5 shows maximum principal stress contours obtained from static analysis 
of straps under combined slosh loads in the $\mathrm{x}$-direction and weight loads in the $\mathrm{z}$-direction.

IMSC. Par on 2003 0! - May-04 18:32:35

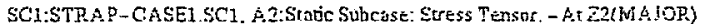

SC1:STR.AP-CASE1.SC1, Aa:Static Subcase: Displacements, Transladional-(NON-LAYERED)

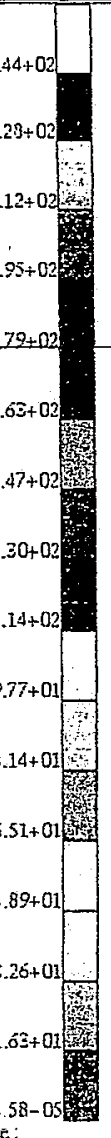

defautr_Fringe:

Fila $3 . \overline{4}+02 @ N d 666$

Min 0.@Nd I

defaut_Deformation:

Wax 1.30+01@1Nd 1248

Figure 5-5 Maximum Principal Stress contours obtained from Static Analysis of the Strap

\subsection{High Cycle vs. Low Cycle Domains}

The onset of high and low cycle fatigue were explained in chapter two. It was stated that if the maximum stress in a component is lower than the yield strength of the material, the component fails under high cycle fatigue. This means that the life cycles to failure is greater than 100,000 cycles. In contrast, when the maximum stress is higher than the yield 
strength, the component fails under low cycle fatigue with life between 100 to 100,000 cycles. As a matter of fact, knowing the failure mechanism before running the fatigue analysis is very important. This is because in the high cycle region, using parameters based upon the S-N curve yields conservative results. Similarly, in the low cycle region, using parameters based upon the $\varepsilon-\mathrm{N}$ curve is preferred. The S-N curve for the chosen material of strap, SAE 1006, is presented in Figure 5-6.

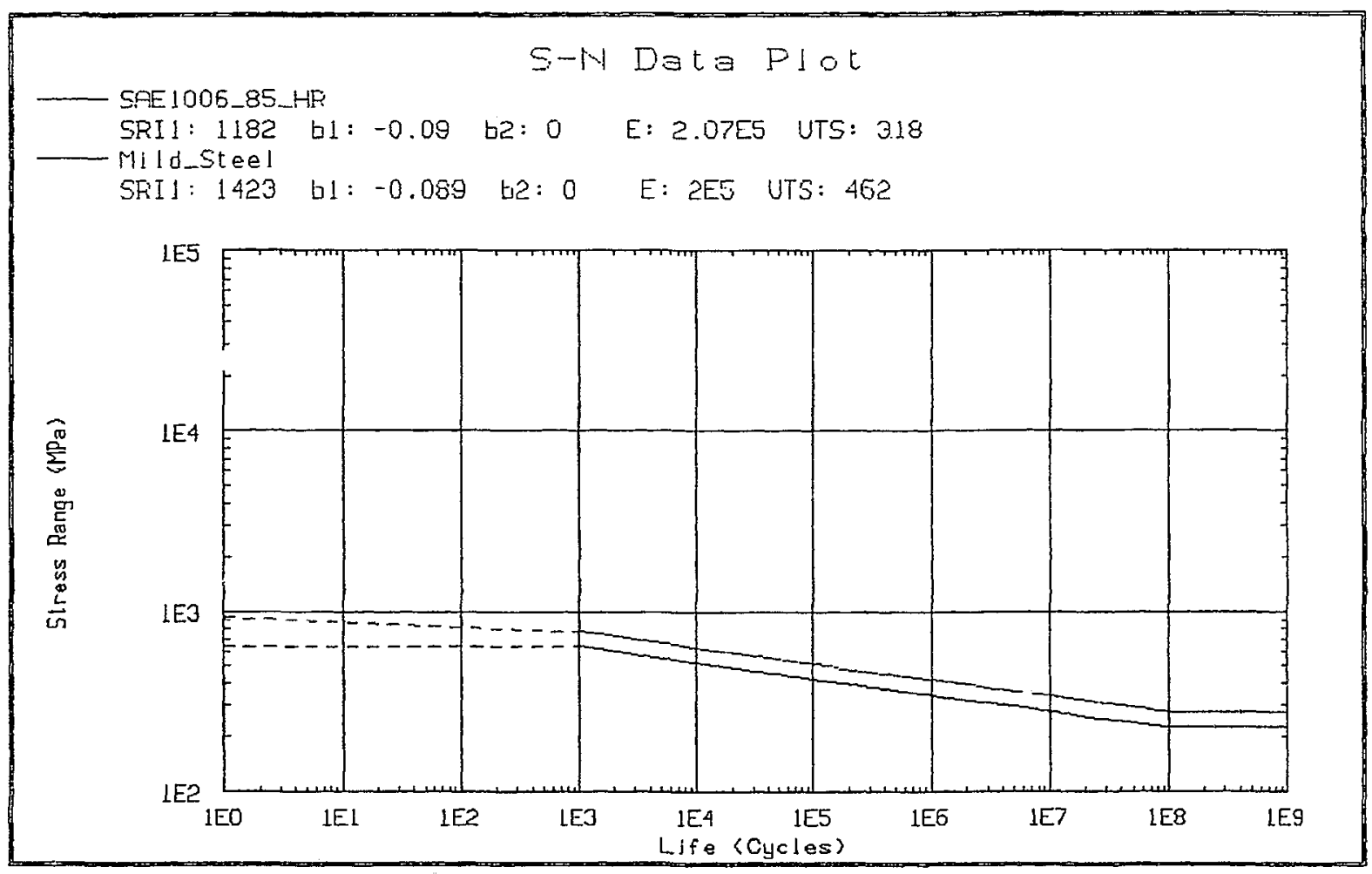

Figure 5-6 The S-N curves of SAE-1006 and Mild Steel

The maximum principal stress obtained from static analysis is $244 \mathrm{MPa}$ while the maximum ultimate stress for the SAE 1006 is $318 \mathrm{MPa}$. Since the maximum principal stress is less than the yield point and lies within the stress range, using the $S-N$ curve would result in the prediction of conservative fatigue life. The maximum principal stresses are 
therefore used as input data in the numerical fatigue analysis.

\subsection{Loading History}

The loading history for the fuel tank strap in the fatigue analysis has been obtained numerically from sloshing simulation, which is shown in Figure 5-7. In gereral, the range of any loading history is from -1 to +1 . For a full cumpression loading, which is the case here, the minimum and the maximum load value should be between zero and -1 . In the sloshing simulation, the applied load on each strap was increased up to a maximum value of $322: 2=161(\mathrm{~N})$. Hence, the true loading history has a minimum and a maximum value of $0 \mathrm{~N}$ and $-161(\mathrm{~N})$ respectively. However, in FEA, the curve defining the loading history is normalized (i.e., the true loading history is divided by the maximum value of $161(\mathrm{~N})$. The static stress resulting is then superimposed with the normalized loading history.

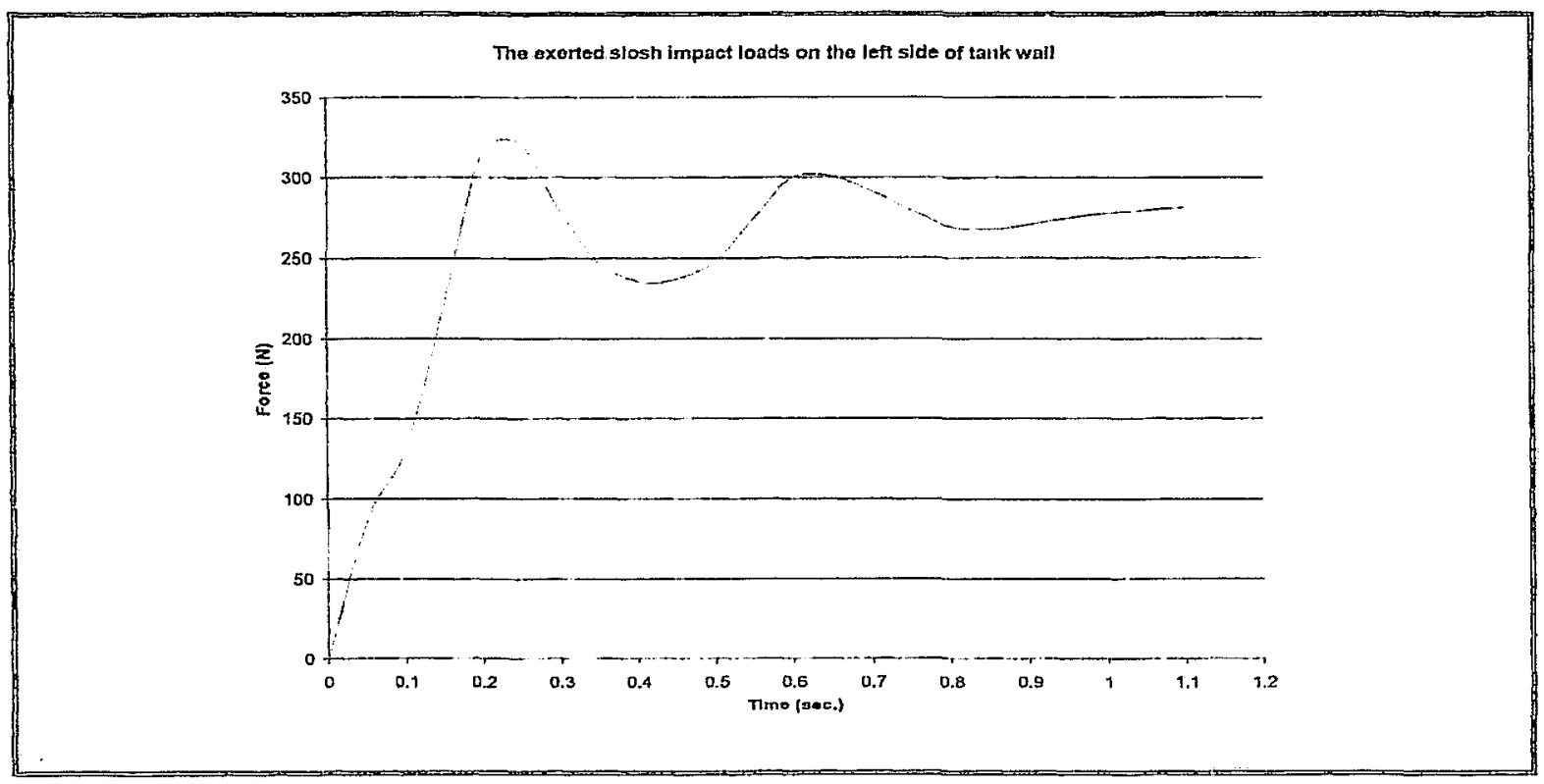

Figure 5-7 Loading History obtaineờ from Sloshing Simulation. 


\subsection{Fatigue Life Estimation of the Strap}

The final step involves combining the scaled loading history and the S-N curve. The loading history is converted to constant blocks of stress amplitudes such that it can be used on the S-N curve. This conversion is done from the rain-flow cycle counting process $[5,11,38]$. The rain-flow cycle counting is an algorithm commonly used in fatigue whereby an irregular load history is converted to blocks of constant amplitude. All the steps herewith mentioned are included in MSC.FATIGUE [5].

\subsection{Simulation Results}

In summary, the maximum principal stress of $244 \mathrm{MPa}$ from static analysis is superimposed with the loading history. The fatigue life (cycles to failure) is then evaluated from the accumulation of damage. Figure 5-8 shows the fatigue life contour in the strap.

The minimum life cycles to failure is shown in a $\log$ scale on the bottom right corner of the Figure 5-8, which is 6.63 . The weakest spot is around the fillet curves of the strap. The minimum life cycles to failure is $10^{6.63}$ which is the inverse logarithmic of the value shown in figure $5-8$, equal to $4,265,000$ cycles. The maximum stress located on the top or bottom surface of the holes (i.e., areas in contact with the washer) were ignored in the analysis. 


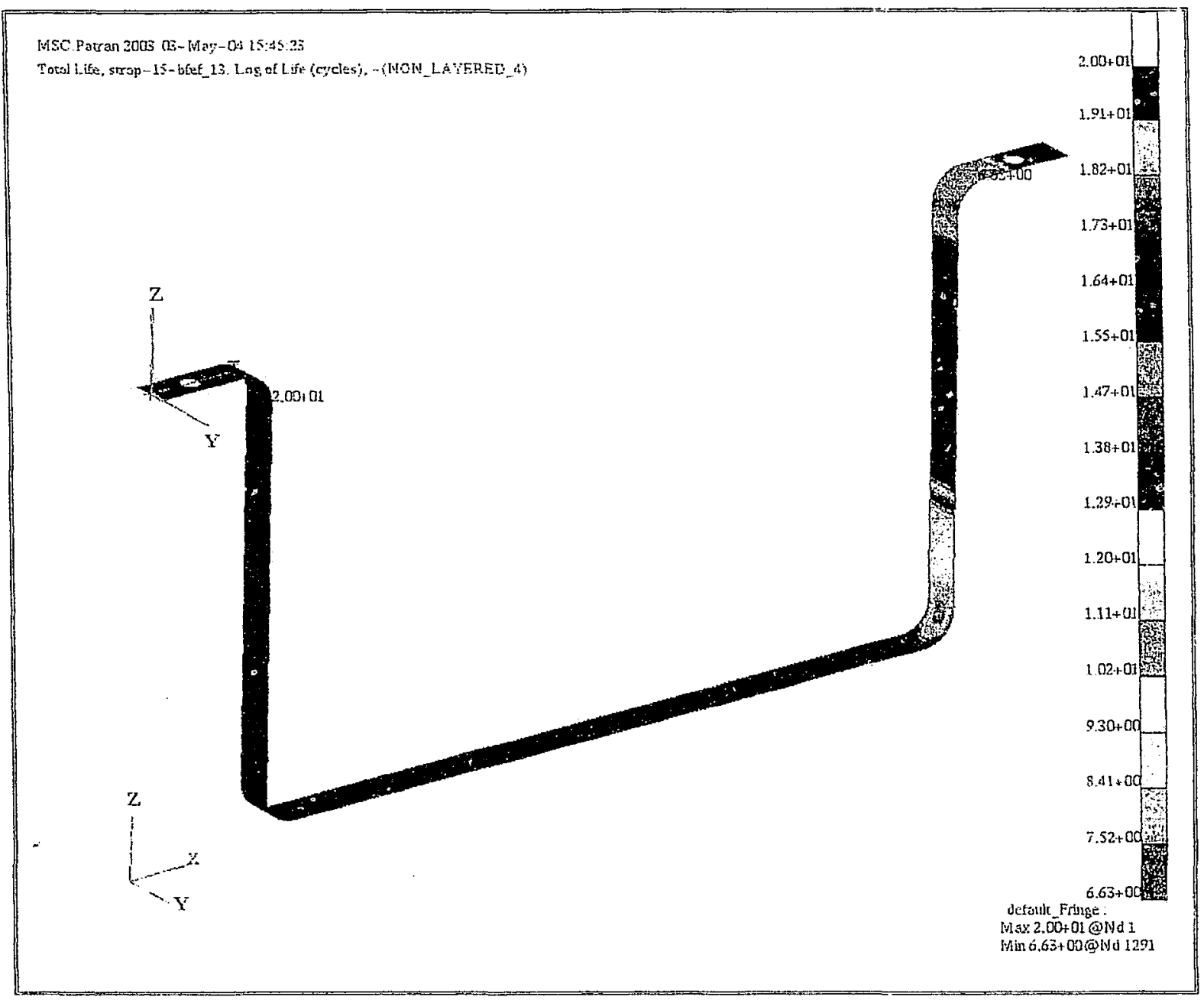

Figure 5-8 Fatigue life contour of the Strap.

\subsection{Results Summary}

The life predicted from the numerical fatigue analysis is $4,265,000$ cycles. As expected the fuel tank strap life is in high cycle range. This result has been calculated just using the slosh loads exerted on the fuel tank in an accelerating car. But in practice, however, many other parameters and functions are involved such as corrosion stress, fretting stress, noise and vibration applied from other components. multi axial acceleration 
of car on the road, and etc.

Although, the fuel tank strap seems to be a simple structural component in the car, its role is very important from a safety viewpoint. If the fuel tank strap is separated due to fatigue during vehicle operation, it will cause the tank to be unsupported thus increasing the risk of a fuel leak and making a dramatic event. For this reason, safe-life design has to be considered in fuel tank strap design for long life more than other components even fuel tank. 


\section{CHAPTER-6 CONCLUSION AND RECOMMENDATIONS}

\subsection{Conclusion}

The present investigation focused on three major parts:

1. Numerical sloshing simulation in a fuel tank

2. Equivalent mechanical model for sloshing simulation

3. Fatigue analysis of fuel tank straps

In part one, it is attempted to show that a certain practical solution to the non-linear sloshing problem in road containers can be achieved using numerical simulation techniques. The finite element (FE) numerical approach yielded the transient responses of the liquid in fuel tanks subjected to braking acceleration. These transient responses were achieved by FE solution of the Navier-Stokes, continuity, and free surface equations using the volume of fluid (VOF) method and donor-acceptor technique. Furthermore, the dynamic responses of the liquid were found in terms of the main sloshing parameters, such as free surface locations, hydrodynamic pressures, and slosh forces. The slosh forces in baffled and un-baffled fuel tanks were compared and the effect of baffles on damping of wave loads were investigated. In summary, there were two major contributions in this part:

1. Development of the numerical modeling of a baffled partially filled rectangular tank to simulate sloshing during accelerating of vehicle.

2. Development of the numerical sloshing simulation of an un-baffled partially filled rectangular tank and comparison of the results with a baffled tank and the effect of baffles in damping slosh waves. 
In part two, special attention was given to the development of equivalent mechanical models, such as the pendulum system, for the numerical solution of sloshing inside rectangular tanks. The pendulum models can simulate the impact loads exerted on the wall of the exited tank much faster than FE simulation. Also, transferring of all other functions of the car to a simple pendulum model is easier than to a continuously deformable medium such as liquid fuel. The obtained results from both FE simulation and pendulum models show good agreement. The contribution in this part was the development of an equivalent pendulum model for non-linear liquid sloshing simulation in rectangular tanks.

In part three, which was the main purpose of this study, the fatigue analysis of the fuel tank straps based on the FE model and slosh loads was conducted. The FE based fatigue analysis was used to locate the critical point of crack initiation and to predict the life in the strap system. For the computational approach dissimilar FE models were built, for combined loading cases. Also, separate FE analyses were conducted in each loading cases: static and fatigue. The static analyses were conducted prior to fatigue and the stress and strain results were then used as input data to fatigue life prediction. The simulation results for the slosh loading case yielded a life of $4,265,000$ cycles.

\subsection{Recommendations for future work}

As stated previously, the sloshing simulation results were verified by an equivalent mechanical model. However, it should be noted that experimental results are needed to validate the numerical results accurately. Hence, it is recommended to design and set up an 
apparatus in the laboratory to simulate the liquid sloshing in an excited fuel tank and to measure the liquid parameters during sloshing. The calculated fatigue life of fuel tank straps herein is based on only the liquid slosh impact loads in braking-acceleration situation of a car, but this is not just the source of stress. There are other sources of the stress applied on the straps such as corrosion stress, fretting stress, noise and vibration applied from other components, multi axial acceleration of the car on the road, etc. Hence, another recommendation for future research is to consider these stress sources in life estimation of straps. 


\section{References:}

[1] El-Sayed, M.E.M., and Lund, E.H., "Structural Optimization with Fatigue Life Constraints", Engineering Fracture Mechanics, vol. 37 (6), pp.1149-1156, 1990.

[2] Popov, G., Sankar, S., "Dynamics of liquid sloshing in baffled and compartmented road containers", Journal of Fluids and Structures, vol. 7, pp.803-821, 1993.

[3] Popov, G., "Dynamics of liquid sloshing in road containers", $\mathrm{PhD}$ Thesis, Concordia University, Canada, 1991.

[4] The National Highway Traffic Safety Administration, officially announced March $10,2000$.

[5] MSC.Fatigue, Encyclopedia. Los Angeles (CA, USA): MacNeal Schwendler Corporation, 2003.

[6] MSC.Nastran, Computer Software. Los Angeles (CA, USA): MacNeal Schwendler Corporation, 2001.

[7] Celebi, M.S., Akyuldiz, H., "Nonlinear modeling of liquid sloshing in moving rectangular tank", In Ocean Eng. vol. 29, pp.1527-1553, 2002.

[8] Pilipchuck, V.N., Ibrahim, R.A., "The Dynamics of a Non-linear system simulating liquid sloshing impact in moving structures", Journal of Sound and Vibration, vol. 205 (5), pp.593-615, 1997.

[9] Ikeada, T., Nakagawa, N., "Non-linear Vibrations of a Structure caused by Water Sloshing in a Rectangular Tank", Journal of Sound and Vibration, vol. 201(1) pp. 23-41, 1997.

[10] Wiesche, S., "Computational Slosh Dynamics: theory and industrial application", Computational Mechanics, vol.30, pp.374-387, 2003.

[11] Diether, G.D., "Mechanical Metallurgy", Third edition, Mc.Graw Hill, Boston 1986.

[12] American Society for Testing and Materials: "Metal Fatigue Damage-Mechanism, Detection, Avoidance and Repair", ASTM Spec. Tech. Publ. 495, 1971.

[13] Fatigue Testing, "Metals Handbook", vol. 8, pp. 361-435, 9th ed., American Society for Metals, Metals Park, Ohio, 1985. 
[14] SAE International, SAE Fatigue Design Handbook, Third Edition, AE-22, Prepared under the auspices of the SAE Fatigue Design and Evaluation Committee, 1997.

[15] Ellyin, Fernand, "Fatigue Damage, Crack Growth and Life Prediction", New Delhi, Chapman \& Hall, 1997.

[16] Hirt, C.W., Nichols, B.D., "Volume of fluid (VOF) method for the dynamics of free boundaries" J. Comput. Phys. vol.39, pp.201-225, 1981.

[17] Sandor, B. I.: "Fundamentals of Cyclic Stress and Strain", The University of Wisconsin Press, Madison, 1972.

[18] Sines, G. and J.L. Waisman (eds.): "Metal Fatigue" McGraw-Hill Book Company, New York, 1959.

[19] Goodman, J., Mechanics Applied to Engineering. Longmans, Green and Co., 1899.

[20] Gerber, T. L., and Fuchs, H. O., "Analysis of Non-propagating Fatigue Cracks in Notched Parts with Compressive Mean Stress," Journal of Materials. JMLSA, vol.3 (2), pp.359-379, 1968.

[21] LaPointe, N. R., "Monotonic and Fatigue Characterizations of Metals," SAE Technical Paper, No. 820679, pp.23-37, 1982.

[22] Ruiz, C., Koenigsberger, F., "Design for Strength and Production", Grordon and Breach Science Publishers, Inc., New York, Pages 106-120, 1970.

[23] Junivall, R.C., "Engineering Considerations of Stress, Strain, and Strength", McGraw-Hill Book Company, New York, Chapters 11 to 16, 1967.

[24] Graham, J.A., "Fatigue Design Handbook", Society of Automotive Engineers, New York, 1968.

[25] Heywood, R.B., "Designing Against Fatigue of Metals", Reinhood publishing corporation, New York, 1962.

[26] Sors, L., "Fatigue Design of Machine Components", Pergamon Press, New York, 1971.

[27] Abramson, H.N., (editor) "The Dynamic Behaviour of Liquids in Moving Container", NASA, SP 106, 1966. 
[28] Shin, S., Lee, W., " Finite element analysis of incompressible viscous flow with moving free surface by selective volume of fluid method", International Journal of Heat and Fluid Flow, vol.21, pp.197-206, 2000.

[29] Wu, G.X., Ma, Q.A., Eatock Taylor, R., "Numerical simulation of sloshing waves in a 3D tank based on a finite element method", Appl. Ocean Res. vol.20, pp.337$355,1998$.

[30] Wu, G.X., Eatock Taylor, R., "Finite element analysis of two-dimensional nonlinear transient water waves", Appl. Ocean Res. vol.16, pp.363-372, 1994.

[31] Choi, H.G., "A study on segregated finite element algorithms for the Navier-Stokes equations", Ph.D. Thesis, Seoul National University, 1996.

[32] FLUENT Manual. FLUENT software.

[33] Floryan, J.M., Rasmussen, H., Numerical methods for viscous flows with moving boundaries. Appl. Mech. Rev. vol.42, pp.323-341, 1989.

[34] Harlow, F.H., Welch, J.E., Numerical calculation of time dependent viscous incompressible flow of fluid with free surface. Phys. Fluids vol.8, pp.2182-2189, 1965.

[35] Brooks, A.N., Hughes, T.J.R., "Streamline upwind/ Petrov- Galerkin formulations for convection dominated flows with particular emphasis on the incompressible Navier-Stokes equation": Comput. Methods Appl. Mech. Engrg. vol.32, pp.199$259,1982$.

[36] Strandberg, L., "Lateral Stability of road tankers", National Road and Traffic Research Institute, Report \#138. Sweden.

[37] Lamb, H., "Hydrodynamics", Cambridge, Cambridge University Press, 1995.

[38] MSC.Patran, Computer Software. Los Angeles (CA, USA): MacNeal Schwendler Corporation, 2003.

[39] Aliabadi, S., Johnson, A., Abedi, J., "Comparison of finite element and pendulum models for simulation of sloshing", Computer and Fluids, vol.32, pp.535-545, 2003. 


\section{APPENDIX}

\section{"A Pendulum Model for Liquid Sloshing in Cylindrical Tanks"}

In this section a comparison between the simulation results obtained from the pendulum model and a finite element model for sloshing of liquids in horizontal cylindrical tanks (tanker trucks) has been presented by Aliabadi et al. [39]. In this pendulum model (Figure A-1), the fuel is assumed to be a point mass attached to the characteristic arm, R. It has been also assumed that the tanker has a circular cross-section and the characteristic arm is the same as the radius of the cross-section. This pendulum is attached to a non-inertial frame using a frictionless hinge (at point $\mathrm{O}$ ).

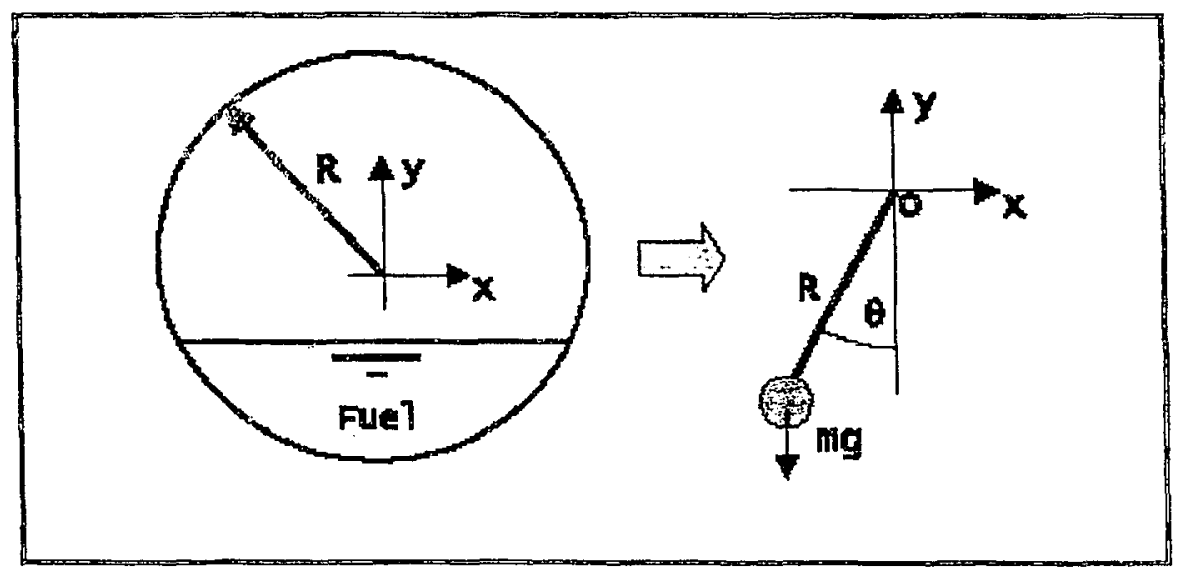

Fig.A-1 Pendulum model for cylindrical tank.

The governing equation of the pendulum motion can be written as

$$
\begin{gathered}
\frac{d \omega}{d t}=\frac{1}{R}(a \cos \theta-g \sin \theta) \\
\frac{d \theta}{d t}=\omega
\end{gathered}
$$


where $\theta$ is the angle of the pendulum with respect to the vertical axis, $\omega$ is the angular velocity, and $\boldsymbol{a}$ and $\boldsymbol{g}$ are the horizontal and vertical accelerations, respectively. The initial conditions associated with equation (A-1) are

$$
\left.\theta\right|_{t=0}=0,\left.\quad \omega\right|_{t=0}=0
$$

By integrating in time of Equations (A-1) and (A-2):

$$
\begin{gathered}
\omega^{n+1}=\omega^{n}+\frac{\Delta t}{2}\left[\frac{1}{R}\left(a \cos \theta^{n+1}-g \sin \theta^{n+1}\right)+\frac{1}{R}\left(a \cos \theta^{n}-g \sin \theta^{n}\right)\right], \\
\theta^{n+1}=\theta^{n}+\frac{\Delta t}{2}\left[\omega^{n+1}+\omega^{n}\right]
\end{gathered}
$$

where the superscript " $n$ " designate the time step and $\Delta t$ is the time increment. Combining Equations (A-3) and (A-4), one can write:

$$
\theta^{n+1}=\theta^{n}+\Delta t \omega^{n}+\frac{(\Delta t)^{2}}{4}\left[\frac{1}{R}\left(a \cos \theta^{n+1}-g \sin \theta^{n+1}\right)+\frac{1}{R}\left(a \cos \theta^{n}-g \sin \theta^{n}\right)\right]
$$

In this equation, the only unknown is $\boldsymbol{\theta}^{n+1}$, which is solved using the Newton-Raphson linearization algorithm.

To measure the accuracy of the pendulum model, sloshing of liquids in a tanker truck during turning has been considered. For this particular case, the radial acceleration is simply equal to the centrifugal acceleration exerted on the tanker truck. If the velocity of the tanker is $v$ and the radius of the turn is $r$ then acceleration of tank is: $a=\frac{v^{2}}{r}$.

Numerical simulations using both the finite element and pendulum models are carried out for a tanker truck having a circular cross-section with one unit in diameter. The horizontal acceleration is set to $0.0408 \mathrm{~g}$. The series of the pictures in Figure A-2 shows 
the results obtained using both the finite element method (solid line) and the pendulum method (dotted line) for fuel elevation of $-0.35,-0.25,-0.15$, and $-0.05 \mathrm{~m}$ measured from the center of the tanker. On each picture, the circle shows the cross-section of the tanker and the elevation of the fuel. The comparison is for the non-dimensional radial force as a function of time (non-dimensional) exerted on the tanker during turning.
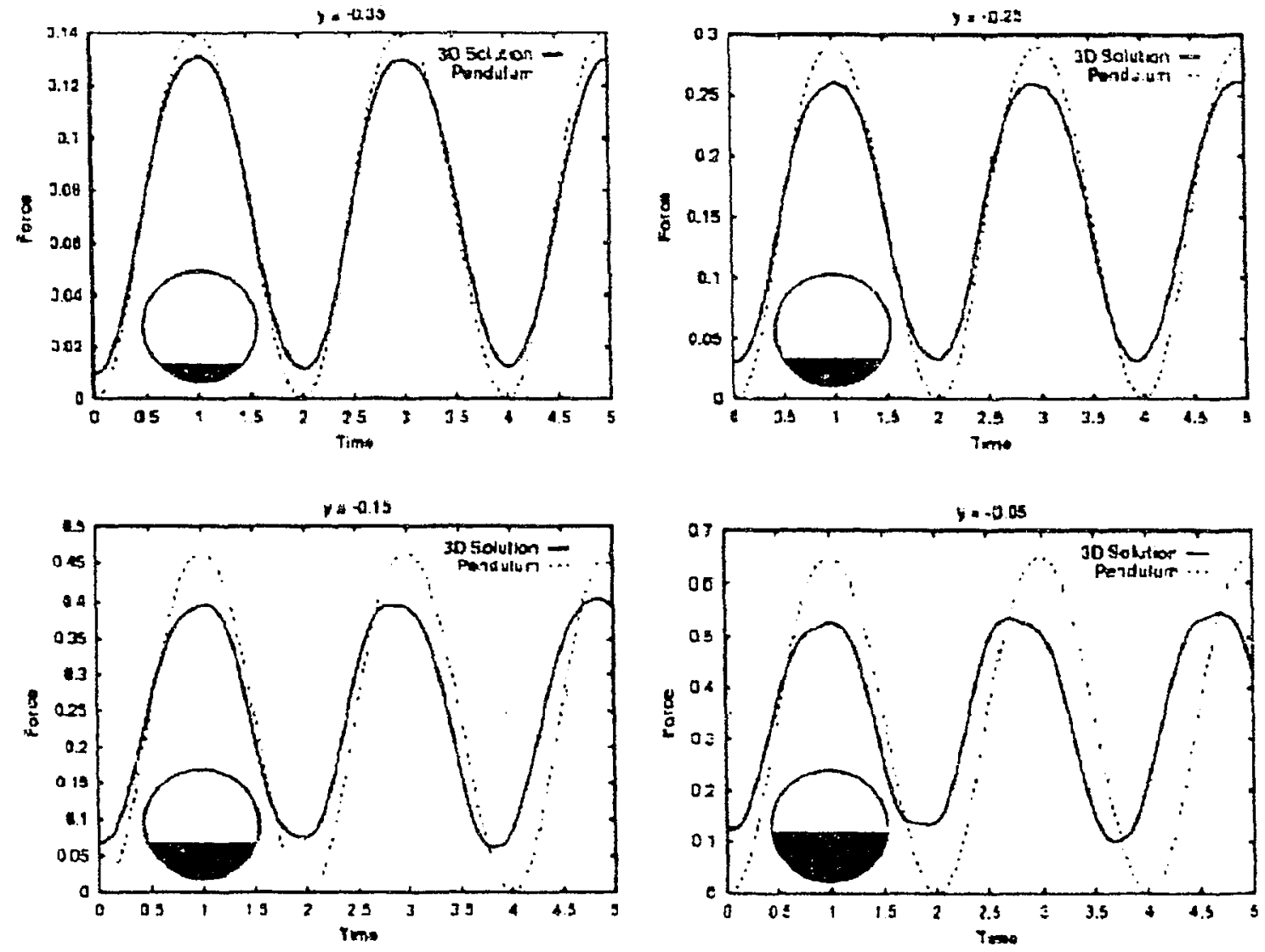

Figure A-2 Radial Force exerted on the Tank during turning. The solid lines show the results obtained using the Finite Element Model and the dotted lines show the results obtained using the Pendulum Model. 
As it is expected, both methods have relatively good agreements when the fuel inside the tanker is low $(y=-0.35 \mathrm{~m})$. The frequency of the sloshing is almost the same for both methods at low and moderate amount of the fuel inside the tanker $(y=-0.35 \mathrm{~m}$ and $y=$ $-0.25 \mathrm{~m}$ ). The amplitude of the force is in good agreements only at relatively low fuel elevations. The difference between both the amplitude and frequency of sloshing is significant when there is a significant amount of fuel inside the tanker $(y=-0.05 \mathrm{~m})$. 
As it is expected, both methods have relatively good agreements when the fuel inside the tanker is low $(y=-0.35 \mathrm{~m})$. The frequency of the sloshing is almost the same for both methods at low and moderate amount of the fuel inside the tanker $(y=-0.35 \mathrm{~m}$ and $y=$ $-0.25 \mathrm{~m}$ ). The amplitude of the force is in good agreements only at relatively low fuel elevations. The difference between both the amplitude and frequency of sloshing is significant when there is a significant amount of fuel inside the tanker $(y=-0.05 \mathrm{~m})$. 